\title{
Identifying Structure in Introductory Topology: Diagrams, Examples, and Gestures
}

\author{
Keith N. Gallagher \\ West Virginia University, kngallagher@mix.wvu.edu
}

Follow this and additional works at: https://researchrepository.wvu.edu/etd

Part of the Science and Mathematics Education Commons

\section{Recommended Citation}

Gallagher, Keith N., "Identifying Structure in Introductory Topology: Diagrams, Examples, and Gestures" (2020). Graduate Theses, Dissertations, and Problem Reports. 7599.

https://researchrepository.wvu.edu/etd/7599

This Dissertation is protected by copyright and/or related rights. It has been brought to you by the The Research Repository @ WVU with permission from the rights-holder(s). You are free to use this Dissertation in any way that is permitted by the copyright and related rights legislation that applies to your use. For other uses you must obtain permission from the rights-holder(s) directly, unless additional rights are indicated by a Creative Commons license in the record and/ or on the work itself. This Dissertation has been accepted for inclusion in WVU Graduate Theses, Dissertations, and Problem Reports collection by an authorized administrator of The Research Repository @ WVU.

For more information, please contact researchrepository@mail.wvu.edu. 
Identifying Structure in Introductory Topology: Diagrams, Examples, and Gestures

\author{
Keith Gallagher
}

\author{
Dissertation submitted \\ to the Eberly College of Arts and Sciences \\ at West Virginia University
}

in partial fulfillment of the requirements for the degree of

Doctor of Philosophy in

Mathematics

\author{
Nicole Engelke Infante, Ph.D., Chair \\ Jessica Deshler, Ph.D. \\ David Miller, Ph.D. \\ John Goldwasser, Ph.D. \\ Geoff Georgi, Ph.D.
}

Department of Mathematics

Morgantown, West Virginia

2020

Keywords: topology, proof, key idea, gesture, structure

Copyright 2020 Keith Gallagher 


\section{ABSTRACT \\ Identifying Structure in Introductory Topology: Diagrams, Examples, and Gestures}

\section{Keith Gallagher}

Despite the prevalence of research in calculus, linear algebra, abstract algebra, and analysis in undergraduate mathematics, the teaching and learning of general topology is a largely unexplored area of research. Although enrollment in courses like linear algebra is often higher than that of topology, the study of students' learning and understanding of topology is of great significance to the Research in Undergraduate Mathematics Education (RUME) community. Courses in topology present many students with their first experience in axiomatic reasoning and explicit interactions with mathematical structure, itself.

I present a thorough case study of Stacey, an undergraduate taking a first course in undergraduate topology. Through the lenses of mathematical structuralism, constructivism, embodied cognition, and commognition, I investigated Stacey's proving behaviors. Papers 1, 2, and 3 present a top-down description of Stacey's behaviors as she sought to identify the key ideas of proofs in general topology. In Paper 1, I described Stacey's proving behaviors using vocabulary borrowed from the literature on problem solving and showed that she used diagrams to arrive at the key idea. In Paper 2, I observed that Stacey seldom produced specific examples, but she reasoned about her diagrams as examples and manipulation of these examples led her to the key ideas of several proofs and to identify appropriate counterexamples when necessary. In Paper 3, I used the theories of embodied cognition and commognition to argue that Stacey's use of diagrams to ground abstract structures in the external world gave her the ability to manipulate those structures spatially, ultimately leading her to the key idea. These three papers are three perspectives on the same theme, each digging more deeply than the one before.

Stacey's behaviors in Papers 1, 2, and 3 describe her search for and investigation of abstract mathematical structures. Stacey's recognition of structure and her ability to work with it helped her to succeed in writing proofs. I conclude this dissertation with suggestions for teaching, including incorporation of the theories of embodied cognition and commognition, as well as directions for future research. 


\section{DEDICATION}

To my parents,

John and Carmela Gallagher,

for their love, support, and patience

and to all my students, past, present, and future,

may you always know you are capable of more than you think. 


\section{ACKNOWLEDGEMENTS}

I owe an enormous debt of gratitude to my advisor, Dr. Nicole Engelke Infante for pushing me, for encouraging me, and for believing in me when I didn't believe in myself. Her door was always open - and I was usually in the doorway. She always made time for me even when she didn't have time for herself, and I always left her office feeling like I could do anything. It's because of her that I had the confidence to finish this work, and I'm excited to continue our work together in the future. I couldn't have asked for a better collaborator.

I want to extend my thanks to Dr. Jessica Deshler for always providing timely feedback on my drafts and for working tirelessly (even when she was exhausted) to support me and my fellow graduate students. Our mutual inability to say "no" to a new project may not have always worked in our favor, but her example always reminded me that I could do a lot more than I thought I could.

Thanks to Dr. Vicki Sealey for first exposing me to the field of RUME and for being so supportive and positive. She provided an excellent foundation that I still think about to this day. She gets a gold star.

I am grateful for Krista Bresock, my writing buddy, fellow graduate student, and teaching partner, for providing her insight and her unwavering support. I am also thankful for all the students I've taught along the way for their inspiration and for reminding me why I was here.

Thanks, finally, to all my family and friends, for their patience and support. They have listened to me ramble and complain about things they surely didn't care about, and they have always waited patiently as I locked myself away for hours, days, or weeks to reach this goal. I only hope that I can repay the kindness and support I have received from all of you. 


\section{TABLE OF CONTENTS}

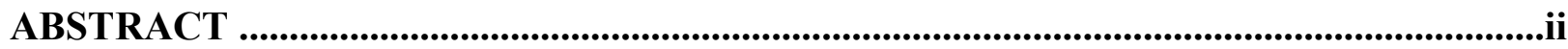

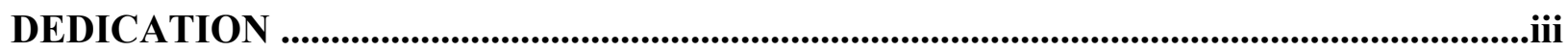

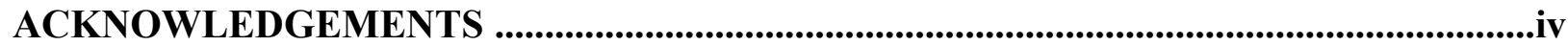

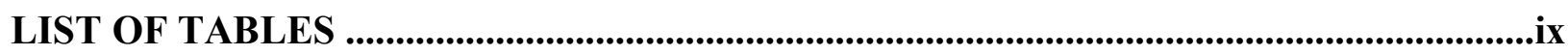

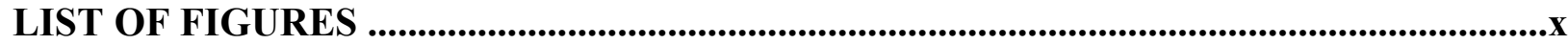

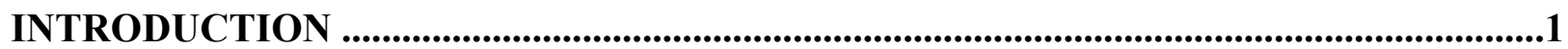

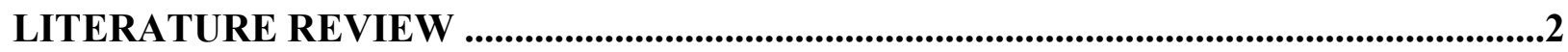

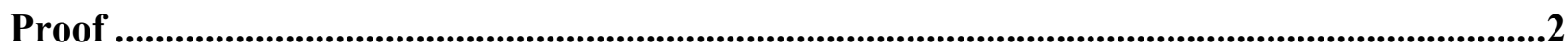

Examples

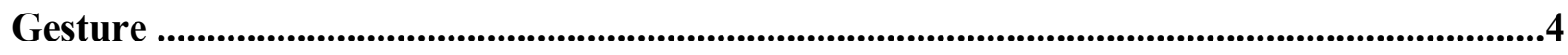

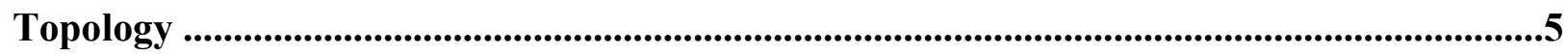

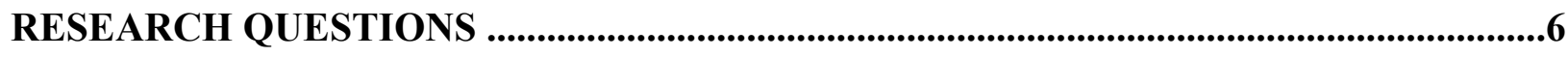

Research Questions for Paper 1 ........................................................................................7

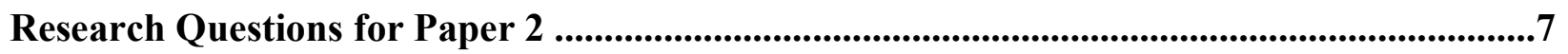

Research Questions for Paper 3 .....................................................................................8

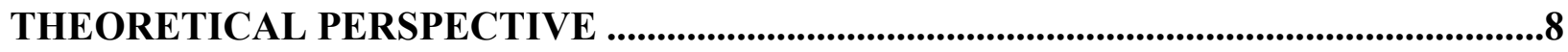

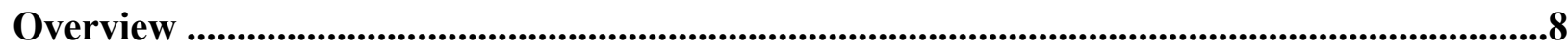

Structuralism and Constructivism .............................................................................................9

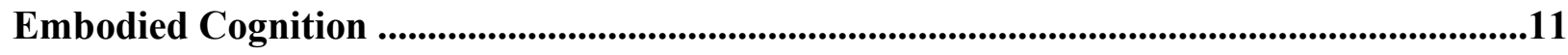

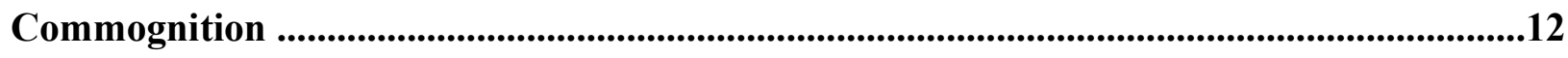

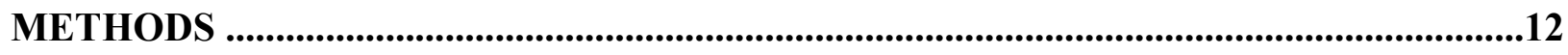

Case Study Methodology .......................................................................................................................12 


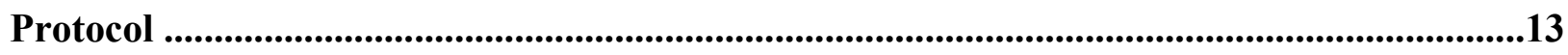

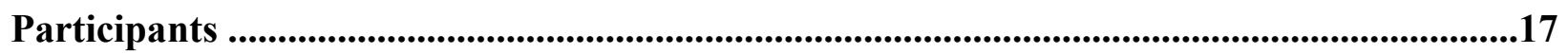

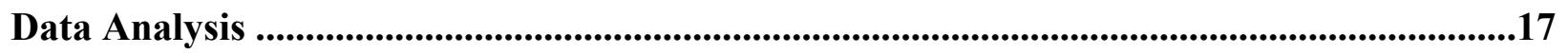

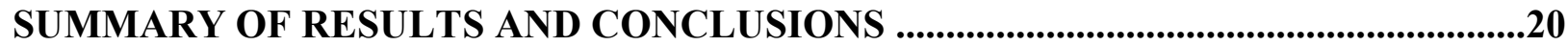

PAPER 1: A CASE STUDY OF UNDERGRADUATES' PROVING BEHAVIORS AND

USES OF VISUAL REPRESENTATIONS IN IDENTIFICATION OF KEY IDEAS IN

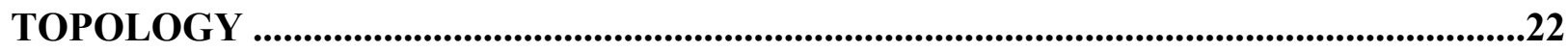

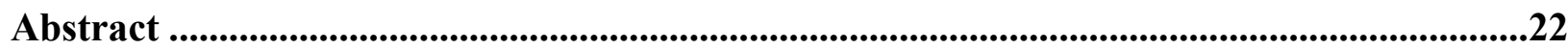

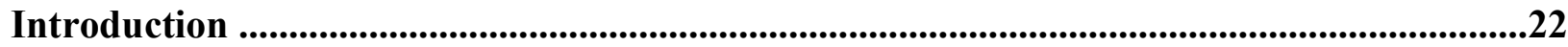

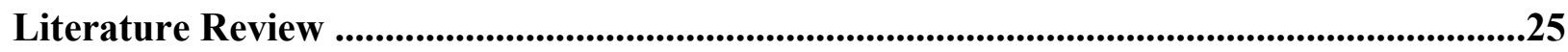

Theoretical Perspective ............................................................................................................28

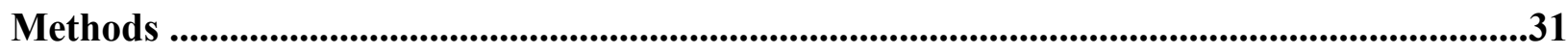

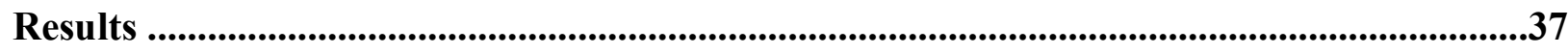

Stacey's Diagrams and Their Role in the Identification of Key Ideas .....................................38

Stacey's Progress Through the Phases of the Multidimensional Problem-Solving Framework

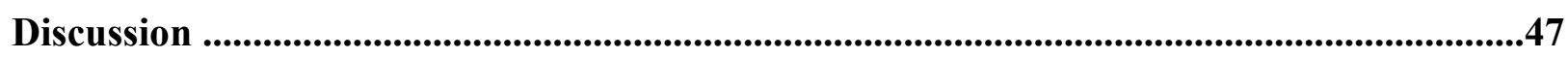

Stacey's Diagrams as an Aid to Recognizing Key Ideas ....................................................47

Stacey's Behavior in the Phases of the Multidimensional Problem-Solving Framework ...........51

Conclusions and Implications for Teaching ….........................................................................53

PAPER 2: STRUCTURAL EXAMPLES: REPRESENTING ABSTRACTNESS IN

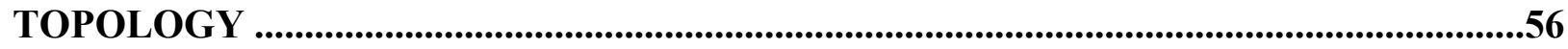

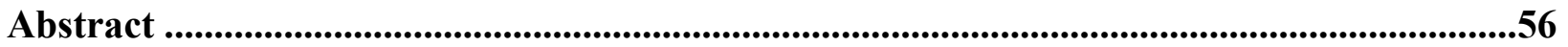




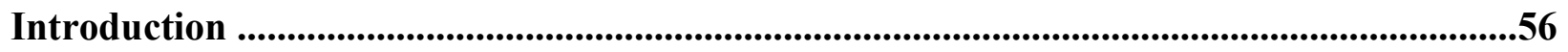

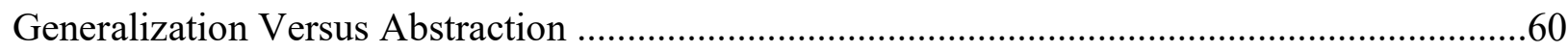

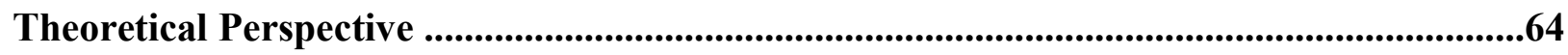

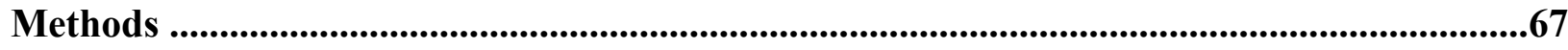

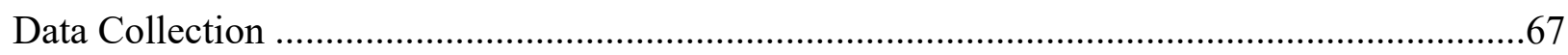

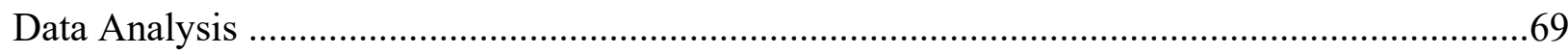

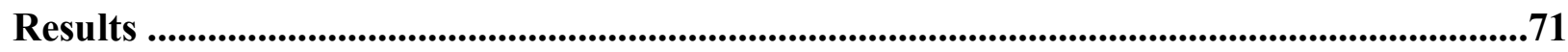

Structural Examples During the Prove Task .................................................................... 71

Structural Examples During the Disprove Task .............................................................. 75

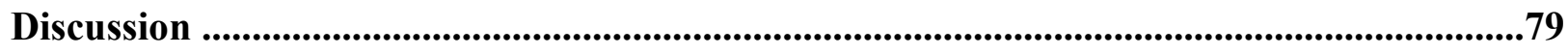

Using Structural Examples to Help Prove True Statements ...............................................80

Using Structural Examples to Identify Counterexamples ..................................................8

Dimensions of Possible Variation and Range of Permissible Change ...................................83

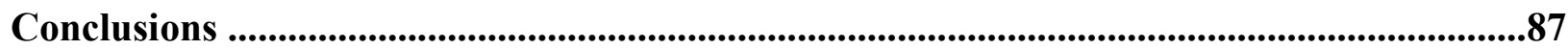

PAPER 3: THE COMBINED EFFECT OF GESTURE USE AND DIAGRAM

CONSTRUCTION ON PROOF WRITING IN TOPOLOGY ................................................89

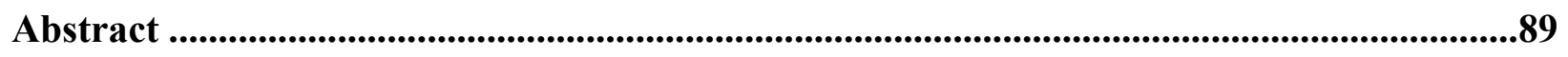

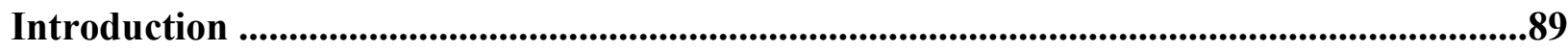

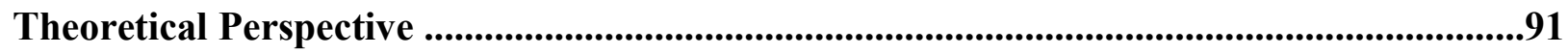

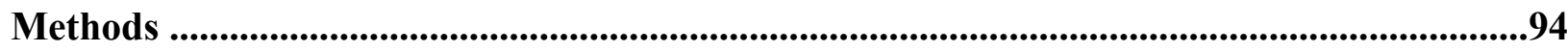

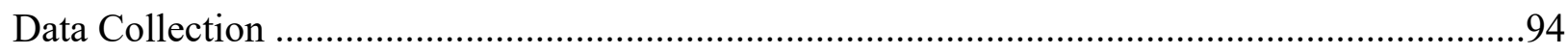

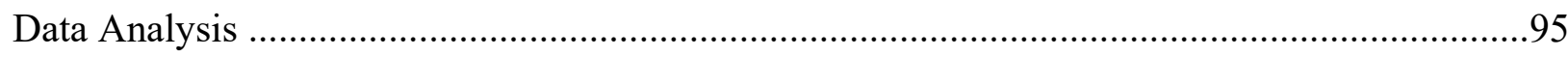

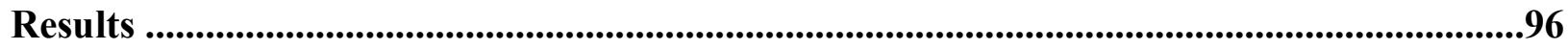


Discussion ........................................................................................................................................104

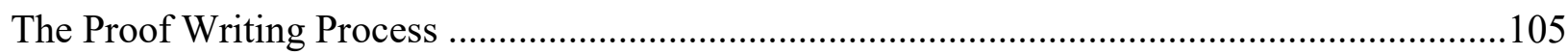

The Role of Embodied Cognition in Stacey's Proving Process ……………………...................107

The Role of Commognition in Stacey's Proving Process ……..................................................110

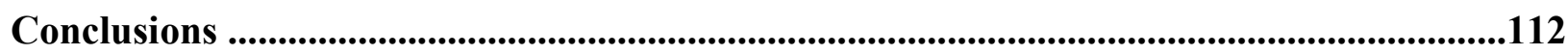

SUMMARY OF PAPERS ...............................................................................................................114

CONNECTIONS AND CONCLUSIONS ............................................................................115

CONTRIBUTIONS TO THE LITERATURE AND IMPLICATIONS FOR TEACHING

FUTURE RESEARCH ..................................................................................................................120

BIBILIOGRAPHY .......................................................................................................................122

APPENDIX A: SESSION 5 (PROVE) CODED FOR PAPER 1 ..............................................127

APPENDIX B: SESSION 5 (PROVE) CODED FOR PAPERS 2 AND 3 ...........................133 


\section{LIST OF TABLES}

Table 1: A list of sessions and the types of proof tasks presented in each ..............................33 


\section{LIST OF FIGURES}

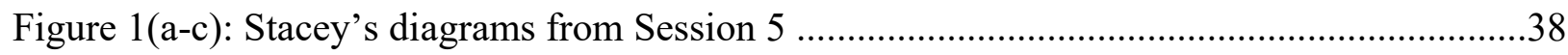

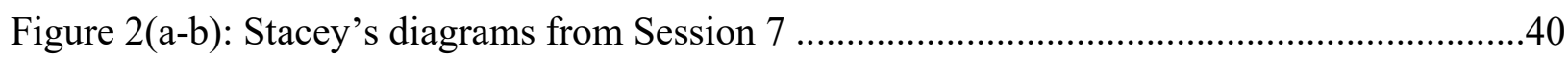

Figure 3: Stacey's diagram representing the Hausdorff condition in Session 9 ...........................41

Figure 4: Stacey's notation from the Prove task in Session 9 .....................................................42

Figure 5: Stacey's diagram representing the problem scenario in Session 9 ...............................42

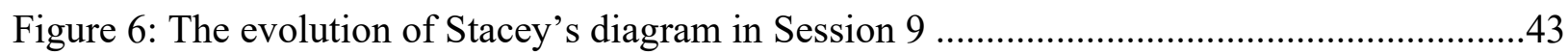

Figure 7(a-c): Stacey's structural examples during the Prove task in Session 5 ...........................72

Figure 8(a-b): Stacey's structural examples during the Prove task in Session 7 ...........................74

Figure 9(a-c): Stacey's structural examples during the Disprove task in Session 5 ......................76

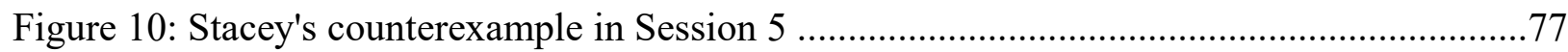

Figure 11: Stacey labeling the false portion of the Disprove prompt in Session 7, beside her

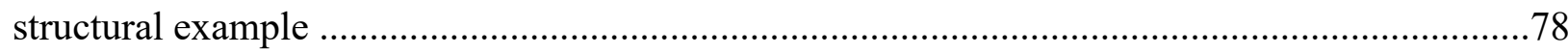

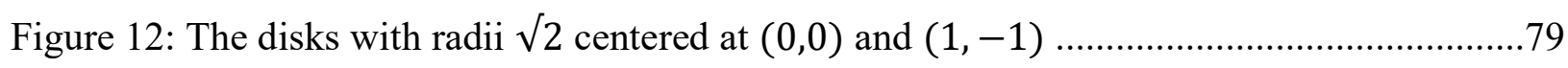

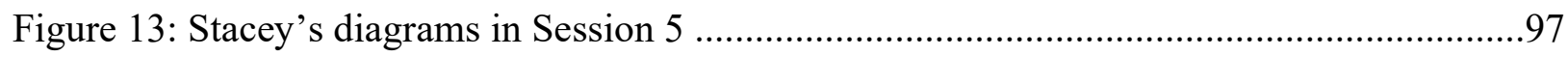

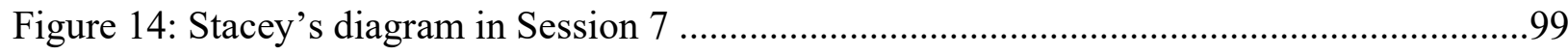

Figure 15: Stacey's metaphoric gestures representing a separation (left) and a connected space

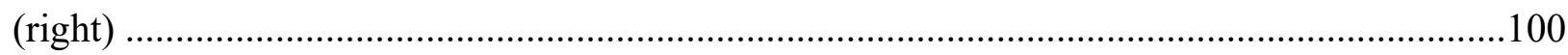

Figure 16: Stacey's notation extracted from the prompt in Session 9 .....................................102

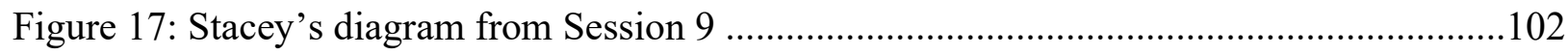

Figure 18: Stacey's proving trajectory in Sessions 5, 7, and 9 .............................................105 


\section{INTRODUCTION}

Topology is the study of properties that remain invariant under twists, stretches, and deformations, without tearing or gluing. The field has strong connections to analysis and geometry, but it is often taught as a stand-alone course or sequence in which students specifically study topological properties like connectedness, compactness, and metrizability as elements of mathematical structure devoid of any specific context (e.g., function spaces). Topology is generally considered an advanced mathematics course, sharing that distinction with areas like abstract algebra and real analysis.

However, while abstract algebra and real analysis have become mainstream topics in the study of undergraduate mathematics education, students' understandings of topology remain largely unexplored, though there has recently been increased interest in studying this phenomenon. Courses like abstract algebra, analysis, linear algebra, and calculus receive thorough treatment in the RUME literature because they are taken by a relatively large number of students. Calculus, linear algebra, and abstract algebra are common requirements for mathematics teacher education programs, and real analysis is commonly required for the completion of a degree in mathematics. Courses in topology, however, are often left as electives, so fewer students generally take these courses.

Despite relatively low enrollment in topology courses, it is vital that we study the teaching and learning of topics in these courses. As Cheshire (2017) pointed out, for many students, their first course in topology is their entry into axiomatic thinking. Students must learn to work directly with mathematical structures, often in the absence of specific examples. This kind of thinking is challenging for students (Berger \& Stewart, 2018, 2019), but it is the essence of advanced mathematical thinking and therefore is worthy of study. 
This dissertation contributes to the literature on undergraduate mathematics education by providing insight into undergraduates' development of understanding of abstract structures. In the three papers in this dissertation, I present an in-depth case study on the behaviors of Stacey, an undergraduate taking a first course in general topology. I examined Stacey's proof writing behaviors from the perspectives of problem solving, examples, and embodied cognition, paying special attention to the diagrams and imagery she used to help her reason through her proofs. My results showed that Stacey's success in proof writing hinged on her recognition of key ideas, and that her recognition of key ideas was supported by her exploration of abstract mathematical structures through grounding them in diagrams and manipulating their resulting spatial properties. I conclude by discussing implications for classroom pedagogical practice and suggestions of directions for future research.

\section{LITERATURE REVIEW}

\section{Proof}

It is well known that students struggle to write and to recognize valid mathematical proofs (Alcock \& Weber, 2010; Azrou \& Khelladi, 2019; Harel \& Sowder, 1998; Iannone \& Inglis, 2010; Komatsu, Jones, Ikeda, \& Narazaki, 2017; Leron, 1983, 1985; Moore, 1994; Selden \& Selden, 2003; Zhen, Weber, \& Mejia-Ramos, 2016). Many potential reasons for students' inabilities to write proofs have been identified: inadequate content-area specific knowledge (Azrou \& Khelladi, 2019; Moore, 1994), difficulty interpreting the logical structure of the statement to be proved (Zandieh, Roh, \& Knapp, 2011), lack of proof techniques and strategic knowledge (Weber, 2001), differences in values and norms between novices and experts (Dawkins \& Weber, 2016; Harel \& Sowder, 1998), and others. With regard to proof validation, Komatsu et al. (2017) noted that students at the secondary level benefited from the activity of 
searching for local counterexamples in the form of diagrams, but that students struggled to produce appropriate diagrams for this purpose. Selden and Selden (2003) suggested that direct instruction in proof validation can improve students' abilities to identify valid and invalid proofs.

Students reason about proof in different ways. Weber and Alcock differentiated students' reasoning into two main categories: syntactic reasoning and semantic reasoning (Alcock, 2004; Alcock \& Inglis, 2008; Weber, 2009; Weber \& Alcock, 2004; Weber \& Alcock, 2009). Syntactic reasoners tend to think about proof using formal definitions, symbols, and logic, while semantic reasoners prefer to use informal methods like diagrams and examples to help them write proofs. Ultimately, while these reasoning methods differ, the proof products resulting from each method are the same. Zazkis, Weber, and Mejía-Ramos (2016) identified productive behaviors used by students who produced graphical arguments to translate their arguments into valid symbolic proofs.

\section{Examples}

A learner's personal example space is "the set of mathematical objects and construction techniques that a learner has access to as examples of a concept while working on a given task" (Sinclair, Watson, Zazkis, \& Mason, 2011, p. 291). Sinclair et al. expanded upon this notion, noting that the example space is not merely a collection of examples but rather has a particular structure dictated by the ease of accessibility of examples to the learner, the connections the learner sees among examples in the space, and the learner's recognition of generality in a particular example. Individual examples have properties known as their dimensions of possible variation (Mason \& Watson, 2008). These are the properties of a mathematical object that can be changed without the object losing its status as an example. Marton and Tsui (2004) argued that 
students may struggle to generate examples of a concept without explicit practice manipulating the dimensions of possible variation of the concept.

Examples are known to give insight into learners' understandings of definitions and processes (R. Zazkis \& Leikin, 2007, 2008). Studies on example use in proof show a variety of different behaviors, including generating examples to clarify a statement or definition, producing an intuitive argument to translate into a proof, or to identify counterexamples (Alcock, 2004; Alcock \& Inglis, 2008; Mason \& Pimm, 1984). It seems that students are generally aware of the purposes and limitations of appropriate example use in proof, but their implementations of examples toward these ends are not always successful (Alcock \& Weber, 2010).

\section{Gesture}

Many researchers establish a direct link between communication and gesture (Alibali \& Nathan, 2007; Alibali et al., 2014; Alibali, Spencer, Knox, \& Kita, 2011; Beilock \& GoldinMeadow, 2010; Bernard, Millman, \& Mittal, 2015; Dargue, Sweller, \& Jones, 2019; Hostetter \& Alibali, 2008; Lakoff, 2012; Lakoff \& Núñez, 2000; McNeill, 1992, 2005; Narayanan, 1997; Roth, 2000, 2001; Sfard, 2009; Straube, Green, Bromberger, \& Kircher, 2011; Varela, Rosch, \& Thompson, 1993). Functional magnetic resonance imaging (fMRI) data have shown that regions of the brain associated with language use and interpretation overlap with regions associated with gesture use and interpretation (Bernard et al., 2015; Straube et al., 2011). Gesture use has been shown to influence strategy choices in problem solving (Alibali et al., 2011) and gestures are often used to facilitate communication (Alibali \& Nathan, 2007; Alibali et al., 2014), though the effect on comprehension is significantly larger for individuals producing gesture versus those observing them (Beilock \& Goldin-Meadow, 2010; Dargue et al., 2019; Hostetter \& Alibali, 2008). 
The connection among thought, communication, and gesture is firmly established in the literature (Barsalou, 2008; Dargue et al., 2019; Hostetter \& Alibali, 2008; Kendon, 2004; Lakoff, 2012; Lakoff \& Núñez, 2000; McNeill, 1992, 2005; Sfard, 2009). The theory of embodied cognition provides an explanation for this connection: namely, that abstract ideas held in the mind are the direct result of our experiences and perceptions of the external world, and in turn these ideas affect our subsequent experiences and perceptions of the world (Barsalou, 2008; Lakoff \& Núñez, 2000; Varela et al., 1993; Wilson, 2002). Sfard's (2009) theory of commognition suggests that thought, verbal communication, and gesture use are all connected and directly influence one another, and it treats thought as a form of intrapersonal communication. This theory accounts for the effects of gesture and communication on the transformation of conception and understanding (Beilock \& Goldin-Meadow, 2010).

\section{Topology}

Topology is commonly taught as a proof-based course in the American university system (Fukawa-Connelly \& Newton, 2014). As such, it requires the ability to work within an axiomatic system, but for many students, their experience of mathematics prior to topology has been heavily conceptual and computational. Cheshire (2017) studied students' transitions into axiomatic thinking through the lens of continuous functions in topology. He reported that some of the participants in his study used the axiomatic structure, itself, to build up their understanding of continuous functions, but others used their concept images to give meaning to definitions (Pinto \& Tall, 2002). Cheshire also reported on obstacles his participants faced in their transitions to an axiomatic understanding of continuous functions, including epistemological obstacles (that is, cognitive conflicts based on deep-seated conceptual beliefs within the individual) and non-epistemological obstacles, such as the use of inappropriate definitions and 
reliance on paradigmatic examples and visual prototypes instead of on formal definitions. Conceptual development in topology is particularly slow; Berger and Stewart (2018) observed that the majority of undergraduates in a topology course were still in the early stages of development of a schema for a topology generated by a basis at the end of the course. In writing topology proofs, recognizing the importance of generality in a schema, connections between concepts, and relevance of schema to a given task were shown to be difficult for students in introductory topology (Berger \& Stewart, 2019).

\section{RESEARCH QUESTIONS}

Over the course of my data collection and analysis, my research questions evolved somewhat. My initial plan was to investigate the following seemingly simple question:

How do students use examples and visualization when writing proofs in topology? Topology, as a branch of mathematics, is not well represented in the research literature in undergraduate mathematics education, and I wanted to help remedy this. However, as I analyzed my data and examined the existing literature on examples and visualization, I realized that there may be many nuanced answers to this question, and I would need to be much more specific.

In my mind, the most problematic part of my research question was the presence of the word visualization. This word means many different things to many different researchers, but in general has a very broad meaning, encompassing not just generic diagrams, but mental images, graphs, gestures, and even logical symbols and notation produced by students, in addition to students' reasoning about imagery produced by others. I was mostly curious about the intuitive images the students generated and used in their reasoning processes, so I thought to study only students' uses of diagrams and mental images. However, as I dug more deeply into my data, I realized that making inferences about student's mental images, to which I had absolutely no 
direct access, would require a much more careful study than the one I had designed. I restricted myself to an investigation of students' diagram use in general topology.

Working under the assumption that the process of proof writing is, in many ways, similar to the process of problem solving, I developed the following initial research questions, which I addressed in Paper 1:

\section{Research Questions for Paper 1}

1. How do students begin their proving processes?

2. How do novice mathematicians proceed through the phases of Carlson and Bloom's (2005) Multidimensional Problem-Solving Framework?

3. When students construct a visual representation (e.g., a diagram), how do they use it? I had not yet addressed my interest in students' example use, and I resolved to do so in Paper 2, and so generated the following research questions:

\section{Research Questions for Paper 2}

4. What kinds of examples do students use to help them write proofs in topology?

5. How do they use them?

The results of my analysis for Papers 1 and 2 showed that Stacey used her diagrams as examples, and furthermore that her reasoning about these examples led her to recognize the key idea of the proof in several cases. It was apparent that Stacey's diagrams had played an important role in this process, but I had yet to identify many of the details of this process.

I returned to thinking about what was happening in Stacey's mind. Although I did not have direct access to Stacey's mental images, I did have an abundance of data from which to make inferences about them. Rather than looking simply at Stacey's diagrams, I chose to investigate Stacey's thinking from three perspectives: (1) her speech, (2) her gestures, and (3) her 
inscriptions (e.g., diagrams and written language and notation). The combination of results from three different sources would allow for triangulation (Yin, 2003b) and provide a stronger argument. I arrived at the following research question for Paper 3:

\section{Research Question for Paper 3}

6. How do students synergize cognition, gestures, diagrams, and communication when writing proofs in topology?

Thus, Paper 1 provides a broad overview of the work in this dissertation, arguing that Stacey used her diagrams as an aid to identifying the key ideas in topology proofs. Papers 2 and 3 give more fine-grained analyses of this result, showing respectively that Stacey reasoned about her diagrams as if they were examples, and that the physical and spatial properties of her diagrams provided the insight that led to her recognition of key ideas.

\section{THEORETICAL PERSPECTIVE}

\section{Overview}

My overarching theoretical perspective for this dissertation incorporates elements of structuralism (Shapiro, 1997) and constructivism (Piaget, 1985) and the theories of embodied cognition (Lakoff \& Núñez, 2000) and commognition (Sfard, 2009). I will present a brief overview of these theories here and the ways they relate to Papers 1,2, and 3 (presented later in this dissertation). In the subsections that follow, I discuss each component of my theoretical perspective in detail.

Mathematical structuralism views mathematics as the study of structures, i.e., connections and relationships. Piaget's theory of constructivism states that learners cannot be given understanding but must construct it for themselves. The main theme of Papers 1, 2, and 3, is Stacey's construction of the ideas of structure necessary to complete proofs in topology. In 
Paper 1, I presented an overview of the process of Stacey's proof productions, highlighting the places where she used diagrams in order to recognize the key ideas of those proofs. In Paper 2, I emphasized that Stacey used her diagrams as structural examples, examples that contained only the skeleton, or basic structure, of what we traditionally think of as examples. Through Stacey's manipulations of the properties of those examples, she was able to recognize key ideas of proofs. In Paper 3, I presented Stacey's structural examples as embodiments of her mental images, allowing her to interact physically with her abstract ideas to make connections between abstract structures and physical ones.

Embodied cognition proposes that cognitive structures are influenced by physical perception, and vice versa. The theory of commognition complements this by recognizing communication (i.e., speech, gesture, and inscriptions) and cognition as the same action. Thus, interaction with a diagram is not merely an external, physical action, but is really a cognitive action. While I utilize these theories most explicitly in Paper 3, I argue that Stacey's creation of diagrams was an act of grounding her mental structures in the physical world. This imbued her mental structures with physical and spatial structure. She was then able to manipulate the physical structures she had created and use her perceptions of these manipulations, together with familiar cognitive schema, to inform her understanding of the abstract structures in question.

\section{Structuralism and Constructivism}

Shapiro (1997) claims that "mathematics is the science of structure" (p. 99). This is the basis of mathematical structuralism: that underlying every example, there is an essential structure, and the purpose of mathematics is to discover that structure. Indeed, mathematics is the study of connections and relationships. From a young age, we learn how numbers relate to one another, and we are taught that combining numbers makes bigger numbers and taking away 
numbers makes smaller ones. We are taught cause and effect, that the occurrence of one event triggers the occurrence of others: the beginnings of logical implication. In advanced mathematics, we write proofs to identify relationships among properties, and we perform calculations to determine the connections among numbers. Resnik (1981) claims that numbers are insignificant on their own; it is only when considered as points or locations within a structure that numbers have meaning. Parsons (1995) supports this claim, further arguing that mathematical objects cannot exist independent of a structure: "[R]eference to mathematical objects is always in the context of some structure, and... the basic objects involved have no more to them than can be expressed in terms of the basic relations of the structure" (p. 74). Mathematical structures can be explicitly described to students, but students cannot be forced to learn. That is, understanding cannot be handed to learners; they must build understanding for themselves (Piaget, 1985; Wadsworth, 1975). According to the theory of constructivism, learning occurs in response to an "intellectual need" (Harel \& Soto, 2017, p. 230), a problematic situation that requires one to learn something new in order to be successful. This process is known as adaptation (von Glasersfeld, 2005). Combining the ideas of constructivism and structuralism leads to the conclusion that, for learners to recognize mathematical structure, they must be placed into a situation in which their success depends upon the recognition of mathematical structure, such as writing mathematical proofs. The creation of this intellectual need stimulates learners to begin the process of adaptation, for if they are to succeed in writing a proof, they must develop an understanding of the underlying mathematical structures at play. 


\section{Embodied Cognition}

The theory of embodied cognition derives from the idea that our mind develops abstract structures in response to external stimuli, and these abstract structures then influence our perception of the world (Goldinger, Papesh, Barnhart, Hansen, \& Hout, 2016; Hostetter \& Alibali, 2008; Lakoff \& Núñez, 2000; Radford, 2009; Varela et al., 1993). Cognitive schema are one such type of abstract structure used to make sense of the physical world, and they are derived from our experiences within it (Lakoff, 2012; Lakoff \& Núñez, 2000). Schema are mental constructs that act like templates for general types of concepts and processes; when certain concepts are perceived to be similar, the same schema can be used to reason about them. The Container schema, for example, consists of an interior, an exterior, and a well-defined boundary separating the two. This schema can be used to reason about many different situations, such as being "outside the building," "in the mathematics department," or "in the set of real numbers." All of these are not literal containers but can be reasoned about using the same general structure represented by the Container schema.

Many cognitive schemas exist and are commonly used in mathematics (Lakoff, 2012). Indeed, the theory of embodied cognition is highly applicable to the field of mathematics (Lakoff \& Núñez, 2000). The concept of number originates in counting objects, and even the more esoteric branches of advanced mathematics often find some application to the physical world. The unbreakable bond between the abstract and the physical often manifests itself in the use of gestures during communication. Gesture use can facilitate communication, demonstrate understanding, transform knowledge, simulate actions, and even affect decision-making (Alibali \& Nathan, 2007; Alibali et al., 2014; Alibali et al., 2011; Barsalou, 2008; Beilock \& GoldinMeadow, 2010; Comi \& Eppler, 2011; Dargue et al., 2019; Hostetter \& Alibali, 2008; Kendon, 
2004; Lakoff, 2012; Lakoff \& Núñez, 2000; McNeill, 1992, 2005). Thus, gesture use provides a rich source of data for the study of students' understanding and thinking about mathematics.

\section{Commognition}

Sfard's $(2001,2009)$ theory of commognition states that communication and cognition are two parts of a larger whole. Commognition is regarded as the combination of thinking and communicating, and argues that it is impossible to do one without doing the other (Sfard, 2009). Furthermore, in this context, communication is not limited to speech but encompasses gesture, symbol, and diagram use as well. This accounts for the relatively common experience of recognizing a previously unnoticed error in reasoning while explaining that reasoning to someone else. Given that thinking and communicating are seen as two sides of the same coin, the theory of commognition also defines thinking as a special form of communication, that is, communication with oneself (Sfard, 2001, 2009). Thus, observing the combination of an individual's speech, gesture use, and inscriptions can provide keen insight into their thought processes and understandings of concepts, even in the case where the individual is simply communicating with themselves.

\section{METHODS}

\section{Case Study Methodology}

I approached my data with a descriptive case study methodology (Cohen, Manion, \& Morrison, 2013; Hamilton \& Corbett-Whittier, 2014; Yin, 2003a, 2003b). There were three primary reasons for the selection of this approach. First, topology is an upper-level course whose primary enrollment is majors in mathematics, and for them it is only an elective. Thus, I had reason to expect that the number of students enrolled in the class would be small, and almost certainly some students in the class would decline to participate in my study. Therefore, I 
anticipated that I would have a very small sample size from which to collect data, so I chose to perform a detailed analysis on those students who did participate. Second, "The case study is the method of choice when the phenomenon under study is not readily distinguishable from its context" (Yin, 2003a, p. 4). The overarching goal of my research was to identify the ways that undergraduates utilized visual representations and examples in the context of writing proofs in general topology. Clearly, the context in which my research was conducted was essential to my ability to address my research questions. Third, "Case studies are the preferred strategy when 'how' or 'why' questions are being posed" (Yin, 2003b, p. 1). The nature of my research questions was not such that quantitative methods were appropriate, as I was primarily interested in describing the nature of phenomena, not how frequently they occurred.

\section{Protocol}

My intent was to capture as authentic an image of student behavior as I could. One-onone structured or semi-structured interviews can be stressful for students, and I was concerned that this type of environment might lead students to behave in ways they normally would not have behaved in order to "say what I wanted to hear." In an attempt to minimize this sort of behavior and the anxiety of my study participants, I advertised my data collection as "Group Study Sessions.” During each Group Study Session, student participants were presented with proof problems related to the content that had been covered in lecture during the preceding week. Participants were then encouraged to collaborate to produce the necessary proofs and counterexamples. They were also permitted to use their textbooks, their class notes, and any other resources they would normally use while studying or working on homework for the class. In this way, I hoped to reduce the participants' anxiety and encourage them to behave in a more 
natural way. I also hoped that the participants would perceive this structure as beneficial to them and therefore be more likely to attend future sessions.

One Group Study Session was held per week (with the exception of the first week of the study). To work within the participants' class schedules, Group Study Sessions were held during the afternoon and were limited to approximately 1 hour in length.

Initially, each Group Study Session was to have a "theme," or a topic to which all the problems in that session related. For example, the theme for Session 1 was "Functions," and the theme for Session 3 was "metrics." Each Group Study Session was to have the following structure:

- Participants would be asked to generate examples of the theme and describe them

- Participants would be given three proofs tasks, one at a time:

○ A "Prove" task, which was presented as true and the students would attempt to prove

○ A "Disprove" task, which was presented as false and the students would attempt to disprove

○ A "Prove or Disprove" task, whose truth value was not specified and the students would attempt to prove or disprove

It quickly became apparent that this was an ambitious structure: during the first two weeks of the study, no group of students was able to progress through the entire structure within the allotted 1hour timeframe. Thus, the structure was modified: starting in Session 4, participants were no longer asked to generate examples at the start of the session. Furthermore, it was no longer expected that the participants should finish all three proof tasks in any given session, merely that they get as far as they could. 
The proof tasks given in each session are listed below, in the order they were presented to the participants:

\section{$\underline{\text { Session } 1}$}

1. Prove: Let $f: S \rightarrow T$, and let $\left\{U_{i}\right\}_{i \in I}$ be a family of subsets of $T$. Prove that $f^{-1}\left(\bigcap_{i \in I} U_{i}\right)=\bigcap_{i \in I} f^{-1}\left(U_{i}\right)$

2. Disprove: If $f: S \rightarrow T$ and $A$ and $B$ are subsets of $T$, then $f^{-1}(A \cup B) \subseteq f^{-1}(A)$.

3. Prove or Disprove: If $f: S \rightarrow T$ and $g: T \rightarrow W$ are surjective functions, then the composition $g \circ f$ is surjective.

\section{$\underline{\text { Session } 2}$}

1. Disprove: Every relation $C$ that is both symmetric and transitive must be reflexive.

2. Prove: Let $f: A \rightarrow B$ be a function. Define a relation $\sim$ on $A$ by setting $a_{0} \sim a_{1}$ if $f\left(a_{0}\right)=$ $f\left(a_{1}\right)$. Show that $\sim$ is an equivalence relation.

\section{$\underline{\text { Session } 3}$}

1. Disprove: Let $\left(x_{1}, y_{1}\right),\left(x_{2}, y_{2}\right) \in \mathbb{R}^{2}$. Then $d\left(\left(x_{1}, y_{1}\right),\left(x_{2}, y_{2}\right)\right)=\min \left\{\left|x_{1}-x_{2}\right|, \mid y_{1}-\right.$ $\left.y_{2} \mid\right\}$ is a metric on $\mathbb{R}^{2}$.

2. Prove: Let $f:(X, d) \rightarrow\left(Y, d^{\prime}\right)$ be a function such that there exists a $y \in Y$ such that for all $x \in X, f(x)=y$. Prove that $f$ is continuous.

\section{$\underline{\text { Session } 4}$}

1. Prove: Let $(X, \mathcal{T})$ be a topological space. Prove that $\emptyset, X$ are closed sets, that a finite union of closed sets is a closed set, and an arbitrary intersection of closed sets is a closed set. 


\section{$\underline{\text { Session } 5}$}

1. Disprove: Let $(X, \mathcal{T})$ be a topological space, and let $A \subseteq X$. Define the boundary of $A$, $\operatorname{Bdry}(A)$, by $B d r y(A)=\bar{A} \cap \overline{C(A)}$. Then $B d r y(A)$ is both open and closed in $X$.

2. Prove: A subset $A$ of a topological space $(X, \mathcal{T})$ is said to be dense in $X$ if $\bar{A}=X$. Prove that if for each open set $O \in \mathcal{T}$ we have $A \cap O \neq \varnothing$, then $A$ is dense in $X$.

\section{$\underline{\text { Session } 6}$}

1. Disprove: Let $\left(X_{1}, \mathcal{T}_{1}\right),\left(X_{2}, \mathcal{T}_{2}\right), \ldots,\left(X_{n}, \mathcal{T}_{n}\right)$ be topological spaces. Then the set $\prod_{i=1}^{n} X_{i}$, together with the collection $\mathcal{T}$ of all subsets of $\prod_{i=1}^{n} X_{i}$ of the form $O_{1} \times O_{2} \times \ldots \times O_{n}$, where each $O_{i}$ is open in $X_{i}$, is a topological space.

2. Prove: Let $(X, \mathcal{T}),\left(Y, \mathcal{T}^{\prime}\right)$ be topological spaces. If $A$ is closed in $X$ and $B$ is closed in $Y$, then $A \times B$ is closed in $X \times Y$.

\section{$\underline{\text { Session } 7}$}

1. Disprove: Let $(X, \mathcal{T})$ be a topological space. Let $A \subseteq X$. If $D$ is a connected subspace of $X$ that intersects both $A$ and $C_{X}(A)$, then $D \cap B d r y(A)=\emptyset$.

2. Prove: Let $(X, \mathcal{T})$ be a topological space. A separation of $X$ is a pair $U, V$ of disjoint open subsets of $X$ whose union is $X . X$ is connected if no separation of $X$ exists. If the sets $C, D$ form a separation of $X$ and if $Y$ is a connected subspace of $X$, then either $Y \subseteq C$ or $Y \subseteq$ D.

\section{$\underline{\operatorname{Session} 8}$}

1. Disprove: $\mathbb{R}$ in the cofinite topology is Hausdorff.

\section{$\underline{\text { Session } 9}$}

1. Prove: If $Y$ is a compact subspace of the Hausdorff space $X$, and if $x_{0}$ is a point of $X$ that is not in $Y$, then there exist disjoint open sets $U$ and $V$ containing $x_{0}$ and $Y$, respectively. 


\section{Participants}

A total of four student volunteers ( 3 undergraduates and 1 graduate student) were recruited from an introductory undergraduate-level course in general topology. Each student volunteer was assigned a pseudonym: Stacey, Tom, and Chris were the undergraduates, and Rachael was the graduate student. Each study participant filled out a demographic survey indicating their academic major(s), minor(s), and any mathematics courses they had taken previously at the university level. Study participants were not required to attend every Group Study Session, so the number of students in attendance each week varied. Stacey was the only student to attend all Group Study Sessions. Due to the abundance of data I collected from Stacey, the results presented in this dissertation focus on her.

On Stacey's demographic survey, she self-reported that she was a Mathematics major with minors in Arabic Studies and in English. Stacey indicated that she had completed the following mathematics courses prior to enrolling in the current course in general topology:

- Plane Trigonometry

- Pre-Calculus with Trigonometry

- Calculus I (Limits and Differential Calculus)

- Calculus II (Integral Calculus, Sequences, and Series)

- Multivariable Calculus

- Introduction to Proof

- Introduction to Linear Algebra

\section{Data Analysis}

Each Group Study Session was video recorded and transcribed. For each of Papers 1-3 presented below, I applied deductive thematic analysis (Braun \& Clarke, 2006), coding for 
different themes in each paper. Deductive thematic analysis was the appropriate choice, as I had an idea of the basic themes I wanted to explore in each case.

For Paper 1, I initially coded for five main themes: orienting, planning, executing, checking, and producing or altering a diagram. The first four of these themes derived from the Problem-Solving Cycle described in Carlson and Bloom's (2005) Multidimensional ProblemSolving Framework, and were operationalized in the same ways. Carlson and Bloom mentioned that, in their study, all participants had used a visual representation or a mental image in the course of their problem solving. Because I was interested in Stacey's uses of visual representations in proof writing, I began with these five themes in order to identify what kind of thinking she was engaged in when she generated visual representations, and to see if I could identify what she was using them for.

As I applied these initial themes to my data, I took notes indicating anytime I saw evidence suggesting what prompted Stacey to produce a visual representation, and anytime I could identify a direct outcome of her reasoning about a visual representation. In some sessions, like Session 2 (in which the main topic was equivalence relations), Stacey produced very few, if any, visual representations. However, in Sessions 1, 5, 6, 7, 8, and 9, Stacey produced and reasoned about visual representations significantly more often. To focus on similar data, I concentrated my attention only on the Prove tasks, and, initially, only on those Prove tasks for which Stacey obtained a complete and correct proof. This restricted my data set to the Prove tasks from only Sessions 5 and 7.

When examining Stacey's behaviors in Sessions 5 and 7, I noticed that Stacey began each Prove task by drawing a diagram to represent the problem, and that after talking about her 
diagram, Stacey expressed an idea that she then tried to incorporate into a formal proof. This prompted me to add one additional theme, recognizing the key idea, and to apply this to my data.

For Paper 2, I wanted to take a more in-depth look at Stacey's example use. I initially coded for the themes of specific examples, generic examples, and algorithmically-generated examples. Specific examples were those examples which were produced in isolation and for a specific purpose, but which gave no impression of generality. Generic examples are specific examples but are also seen by the student to be representative of an entire class of examples, as one might view a generic parabola as representative of the graph of any quadratic function (Mason \& Pimm, 1984). Algorithmically-generated examples are specific examples for which the student appears to have a rule to generate, as one might generate a list of even numbers by starting with 2 and successively adding 2's (Sinclair et al., 2011).

After an initial round of coding with these themes, I noticed that Stacey seemed to reason about some of her diagrams as if they were examples, noting their properties, what was and was not true about them, and reasoning about what would happen to them if the properties were changed. I introduced another theme, structural examples, to represent diagrams such as these which Stacey treated like examples, but which lacked the specificity to constitute a traditional example.

By the time I began to analyze my data for Paper 3, my work on Papers 1 and 2 had already made it apparent that Stacey reasoned about some of her diagrams as structural examples, and that this treatment was an effort to recognize the key idea of a given proof. For Paper 3, I wanted to look more deeply into the mechanism through which Stacey's structural examples led her to recognize key ideas. Appealing to the embodied cognition and commognition perspectives, I coded my data again to identify when Stacey used deictic 
(pointing) gestures, metaphoric (representing abstract ideas) gestures, and iconic (representing concrete objects) gestures, and when she reasoned using schemas (see my Theoretical Perspective for a more thorough treatment of schemas). I then compared the results of this round of coding with the codes I had already assigned for producing or altering a diagram and recognizing the key idea to determine connections between Stacey's speech, gesture use, and diagram use and how those connections led Stacey to discover key ideas.

\section{SUMMARY OF RESULTS AND CONCLUSIONS}

The three papers that follow present a discussion of Stacey's mental construction of the mathematical structures necessary to write proofs in topology (Piaget, 1985; Shapiro, 1997). The discussion is presented in a top-down fashion, with the top layer representing a more surfacelevel explanation of what Stacey's behaviors looked like, and the bottom layer providing a more in-depth view of how those behaviors led to her success in proving.

Paper 1 presents the top layer of the discussion. In this paper, I analyzed Stacey's proving behaviors in terms of problem-solving behaviors described by Carlson and Bloom (2005). I chose to analyze Stacey's behaviors in Group Study Sessions during which she produced a diagram, and I described her diagrams and acknowledged that she used those diagrams to help her identify the key ideas of the proofs.

In Paper 2, I focus more specifically on Stacey's diagrams, themselves. When Stacey produced diagrams, she reasoned about them as if they were examples. These examples, however, possessed only the essential properties necessary to meet a particular definition or situation, but no others. For this reason, I identified these diagrams as structural examples, and I described Stacey's interactions with her structural examples that led her to recognize key ideas to prove true statements and to identify appropriate counterexamples to false claims. 
Paper 3 focuses on embodied cognition and commognition. Embodied cognition proposes that the formation of abstract ideas in the mind is influenced by perceptions of the external world, and subsequently, perceptions of the external world are influenced by the mind. Commognition proposes that communication and thinking are two sides of the same coin. Thus, speech, gestures, writing, and drawing - all of which are part of the act of communication represent cognitive actions. In this paper, I described how Stacey's representation of her mental constructs in the form of diagrams allowed her to manipulate their physical features, thus directly shaping the features of the abstract mathematical objects she held in her mind. Grounding her abstract ideas in diagrams allowed Stacey to manipulate them directly, recognize the consequences of her actions on embodied objects, and translate those results into consequences for the abstract objects in her mind. This manipulation process led Stacey to identify key ideas. 


\title{
PAPER 1: A CASE STUDY OF UNDERGRADUATES' PROVING BEHAVIORS AND USES OF VISUAL REPRESENTATIONS IN IDENTIFICATION OF KEY IDEAS IN TOPOLOGY
}

\begin{abstract}
Visual representations, such as diagrams, are known to be valuable tools in problem solving and proof construction. However, previous studies have shown that simply having access to a diagram is not sufficient to improve students' performance on mathematical tasks. Rather, students must actively extract information about the problem scenario from their diagrams for them to be useful. Furthermore, several studies have described the behaviors of mathematicians and students when solving problems and writing proofs, but few have discussed students' behaviors in the context of proof writing in introductory courses in point-set topology. We present a case study of an undergraduate, Stacey, enrolled in a course in general topology. We describe Stacey's proving behaviors through the lens of a problem-solving framework, and we present Stacey's use of diagrams as an aid to discovering the key ideas of proofs in topology.
\end{abstract}

\section{Introduction}

General topology, also called point-set topology, has its roots in geometry and analysis. While traditional Euclidean geometry concerns itself with congruences and the preservation of distance, topology removes this rigidity and allows for the consideration of more abstract properties such as continuity and connectedness (Gemignani, 2015). Topology is the study of those geometric properties that may be independent of distance, and as such, it gives us tools to identify similarities and differences between objects of different sizes and shapes, such as the torus, the sphere, and the plane. Although topology often ignores the notion of distance, it 
nevertheless describes spatial properties and relationships. It is natural to wonder, therefore, about the utility of visual reasoning strategies when considering problems in topology.

Research into the learning of mathematics has often assumed that students favor one method of reasoning, either visual or analytical (Presmeg, 1986). Implicit in such an assumption is the notion that visualization and analytical reasoning are disjoint processes, but several researchers (Anderson, 1978; Clements, 1982; Presmeg, 1992) argue that this is not the case. Coordination of visual and analytical processes in undergraduates' mathematical thinking led to the development of the Visualization/Analysis (VA) Model (R. Zazkis, Dubinsky, \& Dautermann, 1996), which describes a process by which students might make use of both visual and analytical strategies when solving the same problem by alternating between the two modalities. Stylianou (2002) expanded this model by describing specific behaviors exhibited by mathematicians during visualization steps that informed analytical decisions. Carlson and Bloom (2005) developed the Multidimensional Problem-Solving (MPS) Framework based on their observations of mathematicians solving problems; they identified patterns that include the use of both visual and analytical reasoning strategies.

Carlson and Bloom's work showed that mathematicians tended to begin reasoning about problems visually, often orienting themselves to the problem scenario by drawing a picture or constructing a manipulative. Mathematicians actively used this visual tool, inspecting it for clues which led them to design a plan which they then carried out (using more formal methods) in an attempt to solve the problem, followed by an evaluation of the work they had done to determine the success or failure of their strategy. This framework, however, was built from an analysis of experienced mathematicians. This leads naturally to the question: will undergraduates, who are 
in the beginning stages of learning to prove, display the same pattern of behavior shown by the experts?

Our aim was to answer the following three research questions in the context of writing proofs in a course in introductory topology: (1) how do students begin their proving processes? (2) how do novice mathematicians proceed through the phases of Carlson and Bloom's (2005) MPS Framework? and (3) when students construct a visual representation, how do they use it? We examined the proving behaviors of four students in an introductory course in point-set topology, focusing on the ways these students used visual imagery when reasoning about proof tasks. This paper presents a case study of Stacey, who showed a proclivity for producing visual representations and using them to help her construct proofs. While Stacey demonstrated many of the behaviors described in the MPS Framework, there were key differences in her approach to executing and checking her work compared with the approach used by the experts in Carlson and Bloom's study, and the order in which she passed through the phases described in the Framework was significantly more variable than those of Carlson and Bloom's subjects. Furthermore, we observed that Stacey's productions of visual representations frequently took the form of a diagram representing a key definition in the problem statement, and that her inspection of this diagram led her to identify the key idea (Raman, 2003) of the proof. The results of this study suggest that the generation of visual representations by students in introductory topology may be an effective, low-cost means of improving students' performance on proving tasks. We hypothesize that this may also improve students' interest in topology by making advanced mathematics more accessible to them. 


\section{Literature Review}

The act of proving is a composite of external and internal processes, called persuading and ascertaining, respectively (Harel \& Sowder, 1998). Persuading, the process of convincing others of the truth of a statement, relies on the use of definitions, precise mathematical language, and notation. Convincing arguments for many basic proofs can be written without deep conceptual understanding simply by unpacking definitions. A student who has a general idea of how to write a correct proof of a conjecture simply using logic and formalism is said to understand the procedural idea of the proof (Raman, 2003). Ascertaining, the process of convincing oneself that a statement is true, is a less formal process. A student who has a conceptual reason to believe the truth of a conjecture possesses the heuristic idea of the proof (Raman, 2003). Ideally, proof exercises in the undergraduate classroom will lead students to possess both of these faculties: students should be able to understand and convince others. The key idea of the proof is the idea needed to connect a student's internal understanding to the external argument needed to convince others (Raman, 2003). "A key idea is an heuristic idea which one can map to a formal proof with appropriate sense of rigor. It links together the public and private domains, and in doing so gives a sense of understanding and conviction. Key ideas show why a particular claim is true" (Raman, 2003, p. 323).

Ascertaining first why a conjecture should be true can help the prover discover the key idea of a proof. Empirical data suggest that recognition of the heuristic idea behind a proof facilitates understanding of formal algebraic proofs (Raman, 2001); several authors (Gibson, 1998; Raman, 2003; Weber \& Alcock, 2004) recommended that, when asked to prove a conjecture, students first construct a graphical argument to determine why the statement is true and then base their verbal-symbolic proof on the graphical argument. Zazkis, Weber, and Mejia- 
Ramos (2016) examined how students in a real analysis course translated graphical arguments into verbal-symbolic proofs, and they observed that students who produced multiple graphical arguments tended to be more successful at constructing proofs than students who produced only one graphical argument. This is consistent with results that demonstrate the utility of constructing a graphical argument as an aid in writing proofs (e.g., Alcock \& Weber, 2010). While these studies refer specifically to the use of graphs of functions in proof construction, they provide evidence for the effectiveness of the use of visual representations in proof writing.

To be successful in problem solving, it is not enough to have a visual representation: one must actively seek to extract useful information from it (Booth \& Thomas, 2000; Carlson \& Bloom, 2005; Mason, 1992; Stylianou, 2002; R. Zazkis et al., 1996). For our work, we adapted Dimmel and Herbst's (2015) fairly broad definition of a diagram (originally specified for the context of plane geometry) as "the two-dimensional visual representations that accompany problems (e.g., proof problems, find problems, determine problems)" (p. 155). The issue of the distinction between looking at and using diagrams was raised by Mason (1992), who indicated that "students need to be active in processing images; they need to work on the images, not just look at them" (p. 5). Booth and Thomas (2000) observed that students with better spatial reasoning abilities, including creating, manipulating, and interpreting visual representations, were more likely to obtain correct solutions to problems, regardless of whether those problems were presented verbally or visually. However, they also reported that spatial ability made no difference when students produced their own diagrams, and that all students were more likely to produce an incorrect answer in this case. They argued that this was a consequence of these students producing but not actually using their diagrams in solving problems. 
Presmeg (1986) reported that successful students in high school mathematics were predominantly nonvisualizers (i.e., students with a preference for nonvisual methods). She argued, however, that this may be due to the favor shown to such methods in curriculum design, examinations, and teaching emphasis: "Whether this pattern of thinking is adequate at higher levels in which creative mathematical thought is required, is an open question, but within the constraints of the school system the one-sidedness of nonvisual thinking does not appear to be a disadvantage" (p. 301). Though Presmeg's study seems to show an advantage for students who prefer nonvisual methods of solution, she described a few ways in which visual methods proved useful. Images provided "mnemonic advantages," (p. 302) helping students recall facts and theorems. It was also observed that "imagery which served an abstract function was found to be effective" (p. 302). While Presmeg warned against the "one-case concreteness" (p. 301) of images, which can lead students into erroneous reasoning, she observed that when diagrams were used not to represent a specific case, but rather to represent an abstract idea held in the mind of the student, this could prove beneficial to the student's reasoning. These findings agree with Booth and Thomas' (2000) claim that "visual stimuli are valuable but an over-reliance on imagery in mathematical thinking can limit mathematics performance and difficulties can occur" (p. 171).

When working through problems, students often rely on their concept image (Tall \& Vinner, 1981) rather than on the concept definition (Moore, 1994). "The concept image is something non-verbal associated in our mind with the concept name. It can be a visual representation of the concept in case the concept has visual representations; it also can be a collection of impressions or experiences" (Vinner, 2002, p. 68). In everyday life, people do not often consult with the definitions of the terms we use; rather, we rely on our concept images of 
words like table and car to help us understand and reason. Mathematical problem solving, on the other hand, is what Vinner referred to as a "technical context" (2002, p. 67). In such contexts, consultation with the definition is essential, as the elements of one's concept image which come to mind first - the evoked concept image - may be inappropriate for the given context. "In technical contexts, definitions might have extremely important roles. Not only that they help forming the concept image but they very often have a crucial role in cognitive tasks. They have the potential of saving you from many traps which are set by the concept image" (Vinner, 2002, p. 69). Unfortunately, many students attempt to solve problems using only their concept image (without consulting the concept definition), which can lead to confusion or invalid reasoning. Moore (1994) observed that when students used only their concept images to write proofs, they were often unsuccessful; however, when students used imagery to inform their understanding of the concept definition, the definition became a more effective tool in students' proof constructions.

\section{Theoretical Perspective}

Many studies have investigated factors affecting mathematical problem solving and the behaviors of students and experts when solving problems (Carlson \& Bloom, 2005; Pólya, 1957; Shoenfeld, 1982, 1983; Stylianou, 2002; R. Zazkis et al., 1996). In mathematics and other fields, it is common to refer to certain problems, topics, or tasks as "difficult" or "easy." However, like Lester (1994), we are of the opinion that "problem difficulty is not so much a function of various task variables as it is the characteristics of the problem solver, such as traits (e.g., spatial visualization ability, ability to attend to structural features of problems), dispositions (i.e., beliefs and attitudes), and experiential background (e.g., instructional history, familiarity with types of problems)" (pp. 664-665). That is to say that the difficulty of a task is not something to be 
measured objectively and free from any frame of reference, but rather that difficulty is relative to the individual performing the task.

The goal of this study was to describe students' behaviors during proof construction in general topology. For this reason, we approach our data from the perspective of the MPS Framework (Carlson \& Bloom, 2005). This framework outlines a series of processes, called phases, that problem solvers tend to engage in when solving a problem. These phases are orienting, planning, executing, and checking. Furthermore, this framework, as well as others in the problem-solving literature, recognizes the importance of various attributes of the prover, such as their access to resources and heuristics, their affective dimensions, and their tendency and ability to self-monitor. In our work, we focus on the behaviors our subject demonstrated as she engaged in each of the phases of the MPS Framework. Rather than detail the influence of each of her personal attributes as outlined in the MPS Framework, we call attention specifically to the prover's experience with constructing proofs in topology as a critical factor influencing her behaviors when reasoning about and constructing proofs. While this framework describes the behaviors of expert mathematicians when solving computationally-based problems, we find that the behaviors of novice mathematicians when reasoning about proofs can be broadly classified into similar categories, with subtle differences. This paper highlights the similarities and differences in problem solving behaviors displayed by expert and novice mathematicians, respectively.

We call particular attention to Stacey's production of visual representations in the form of diagrams. Stacey’s diagram construction primarily took place during the orienting phase, identified by "the predominant behaviors of sense making, organizing, and constructing" (Carlson \& Bloom, 2005). It is known that the construction of visual arguments aids in problem 
solving and in the generation of proofs (Alcock \& Weber, 2010; Booth \& Thomas, 2000; Gibson, 1998; Raman, 2001, 2003; Weber \& Alcock, 2004; R. Zazkis et al., 1996), and it appears that students and mathematicians are at least subconsciously aware of this as well: "The drawing of a diagram was not a goal in itself but a means to aid [mathematicians] in gaining more information for the problem situation. Mathematicians anticipated that a figure would provide them with specific information - the drawing of a diagram was not simply a vague step forward in the solution of the problem" (Stylianou, 2002, p. 310). We argue in this paper that the information Stacey anticipated from her diagrams was the key idea of the proof (Raman, 2003).

Following the orienting phase, mathematicians transitioned into the planning phase, which contained a cycle in which the mathematicians would conjecture a possible solution method, imagine applying that method, and then evaluate its effectiveness. This sub-cycle of conjecture-imagine-evaluate repeated until the mathematician settled on an approach that seemed viable, at which point she transitioned into the executing phase. In the executing phase, the mathematician simply carried out the decided-upon plan. This phase was characterized by "making constructions and carrying out computations" (p. 63). In the checking phase, behaviors focused on spontaneous verification of results obtained in the previous phase.

As noted in the MPS Framework, the creation of a visual representation of the problem (or some part thereof) was a common activity during the orienting phase; this was no different for Stacey. As Stylianou (2002) and others argue, such representations are not, on their own, useful. Rather, the individual must make an effort to use their representations to gain additional insights about the problem.

Raman (2003) distinguishes between "private" and "public" elements of mathematics: “By 'private argument' I mean, 'an argument which engenders understanding', and by 'public' I 
mean 'an argument with sufficient rigor for a particular mathematical community"' (p. 321). She also highlights a peculiar epistemological difference between expert mathematicians and students. Students sometimes see proof production as "creating something out of nowhere" (Raman, 2003, p. 322), even if they possess an internal sense that the statement they're proving should be true. For students, connecting a privately held argument (a heuristic idea) with a public argument (a procedural idea) may be a laborious task, if they even see that such a connection should exist. For mathematicians, however, a primary goal is the determination of just such an idea: mathematicians look for a private argument which can be appropriately translated into a public argument. Such an argument is based on the key idea of the proof, the idea which provides the mathematician both with understanding and with the ability to convince others.

\section{Methods}

Student volunteers were recruited from an introductory undergraduate course in topology at a large research university in the United States for a study on undergraduate students' uses of visual representations when writing proofs in point-set topology. Four students (three undergraduate and one graduate) volunteered to participate in the study, and each student attended at least one weekly, hour-long "group study session." Students were not required to attend every session, so the number of participants in each session ranged from one to four. No group study sessions were held during weeks in which the students had an exam in topology. The

first author did not teach the topology course but attended each class session (excluding exams) to know what content had recently been taught. The first author acted as facilitator for the group study sessions. The role of the facilitator was to attend the group study sessions and present problems to the students, prompt students for clarification of their thoughts and statements, and 
move the sessions forward whenever the students seemed to reach an impasse. The role of the facilitator was similar to that of a clinical interviewer, though we refrain from referring to the facilitator as such in this context due to the informal nature of the sessions. As compensation for participating in the study, the facilitator offered extra office hours for participants to receive help with topology.

The design of the sessions was intended to elicit, to the extent possible, the natural behaviors of students when working through a proof task in a homework assignment or on a quiz or test. In each session, time permitting, students were asked to prove one true statement and to disprove a false one. The order in which the two proof tasks were presented varied from session to session. Proof tasks were chosen based on material that had been taught recently in class. Students were encouraged to collaborate, and they were permitted to use their textbooks and class notes. During their first session, each study participant completed a demographic survey indicating their academic major(s), minor(s), and all university-level mathematics courses they had taken previously. This was done to give the authors a sense of the students' prior knowledge of mathematics and proving experience. Students were encouraged to speak aloud as they worked during the sessions; the facilitator occasionally prompted students to vocalize their thinking and asked probing questions. It was also sometimes necessary that the facilitator intervened when the students seemed to be making no significant progress on a problem. Due to time constraints, the students did not always arrive at complete (or correct) solutions to both the "Prove" and "Disprove" problems during the group study sessions. Table 1 below shows which types of tasks were completed in each session. 


\begin{tabular}{|c|c|c|}
\hline Session & Prove & Disprove \\
\hline 1 & $\mathrm{X}$ & $\mathrm{X}$ \\
\hline 2 & $\mathrm{X}$ & $\mathrm{X}$ \\
\hline 3 & $\mathrm{X}$ & $\mathrm{X}$ \\
\hline 4 & $\mathrm{X}$ & \\
\hline 5 & $\mathrm{X}$ & $\mathrm{X}$ \\
\hline 6 & $\mathrm{X}$ & $\mathrm{X}$ \\
\hline 7 & $\mathrm{X}$ & $\mathrm{X}$ \\
\hline 8 & & $\mathrm{X}$ \\
\hline 9 & $\mathrm{X}$ & \\
\hline
\end{tabular}

Table 1: A list of sessions and the types of proof tasks presented in each.

Group study sessions were video recorded and transcribed. The data and conclusions discussed in this paper focus on the behaviors of one undergraduate student, Stacey, because she was the only student present for every session. More specifically, we focus on the results of the Prove tasks and exclude the results from the Disprove tasks due to the distinct types of reasoning employed by Stacey when proving true statements versus disproving false ones. On occasions when Stacey was the only student present, the facilitator's interactions with her were more frequent. There were nine distinct group study sessions and for five of these, Stacey was the only student present.

Due to our interest in describing how undergraduates interact with visual representations (rather than the prevalence or cause of such behaviors), we present our results as a descriptive case study of Stacey's behavior. As Yin (2003b) suggested, we determined that a descriptive case study methodology was appropriate due to the necessity to define our research topic 
broadly. While students' uses of visual representations have been studied extensively, our study is unprecedented due to the specific context of proof-writing in topology. Consequently, there is a need to identify the form(s) such behaviors may take in this context. Our goal is to describe, in detail, a particular set of behaviors exhibited by Stacey.

Initially, extensive notes were taken to identify instances of Stacey producing drawings, generating examples, and writing proofs. During this process, it became evident that Stacey sometimes used drawings to help her visualize definitions or to represent aspects of the problem scenario, and that these drawings seemed to provide her with some insight into the problem or its proof. Based on this observation, the session transcripts were coded using deductive thematic analysis (Braun \& Clarke, 2006) for instances of Stacey constructing or working on drawings related to a definition, instances of Stacey arriving at the key idea of a proof (Raman, 2003), and evidence of Stacey's transitions through the phases of Carlson and Bloom's MPS Framework (2005).

The code working on a diagram was applied any time Stacey drew any portion of a diagram. This included partial or total diagram construction as well as any time during a session that Stacey made a modification or alteration to an existing diagram. Our initial focus was on "diagram construction," but we observed that Stacey sometimes made additions or changes to her diagrams as she attempted to make progress on a proof or when explaining her reasoning aloud. Excerpts coded as recognizing the key idea occurred, at most, once per problem session. This code was used to identify the moment when Stacey vocalized an idea that the authors considered to be central to the proof and that Stacey subsequently attempted to apply formally in a proof. In order for a piece of transcript to be coded as recognizing the key idea, Stacey must have expressed some kind of conceptual understanding of why the proof is true based on this 
excerpt (e.g., in Session 5, "I get it conceptually I think"). In some cases, Stacey's nonverbal behaviors, such as tone of voice and speaking pace, were taken as evidence of the occurrence of a "light bulb moment," a point during Stacey's reasoning at which she suddenly gained a new understanding of some component of a problem. Excerpts in which the code recognizing the key idea was applied can be found in the Results section under the sub-heading Stacey's Diagrams.

We applied a code corresponding to each of the four phases of the Problem-Solving Cycle of the MPS Framework (Carlson \& Bloom, 2005): orienting, planning, executing, and checking. Initial application of each code was in keeping with the descriptions given by Carlson and Bloom, as described in the preceding Theoretical Perspective section of this paper. However, during initial coding, behaviors were identified which were not documented by Carlson and Bloom in their original framework, but which seemed to belong to these categories. These behaviors were incorporated into the four codes identifying the phases of the framework, and the resulting codes are described below. We present a coded transcript excerpt in Appendix A.

Orienting was used to identify any action by means of which Stacey attempted to gain a more thorough understanding of a problem. Actions which indicated Stacey's engagement in the orienting phase of the Multidimensional Problem-Solving Framework included deliberate reading of the problem (we acknowledge that an initial reading of the problem is necessary; we refer here to more thorough scrutiny of the text than a cursory initial reading), extracting notation from the text of the problem (i.e., reorganizing notation from paragraph form into a list or collection) or converting written language into mathematical notation, drawing diagrams, looking up theorems or definitions in the textbook, and asking clarifying questions. It is not necessarily the case that actions such as drawing a diagram or asking a question were always coded as orienting, as the (perceived) intent of the question must be considered. For example, 
Stacey sometimes altered a diagram in order to illustrate her thinking or to answer a question posed by the facilitator. Such actions were not coded as orienting because the perceived intent of the drawing was not to improve Stacey's understanding but simply to explain it to someone else.

Instances in which Stacey expressed consideration of a potential proof strategy or a specific step in a proof were coded as planning. Planning activities were those that expressed ideas regarding Stacey's future actions while proving. Such behaviors were nearly always verbal, and included such things as the identification of a potential proof strategy (e.g., proof by contradiction, proof by contrapositive, etc.), the proposal of a step(s) in a proof, and the preemptive recognition of consequences of actions or properties (e.g., if a subspace $Y$ intersects nonempty open sets $C$ and $D$, then $Y$ will not be connected). The last of these behaviors resembles the conjecture-imagine-evaluate sub-cycle of the planning phase described by Carlson and Bloom. However, Stacey often evaluated the effectiveness of her conjectures by moving into the executing phase and simply trying them out rather than through imagination and mental evaluation.

Any step taken in the production of a written proof was coded as executing. Writing was a necessary condition for an action to be coded in this way. Examples of actions that were coded as executing include declaring elements and sets (e.g., "Let $O_{1}$ and $O_{2}$ be closed sets."), making assumptions and suppositions (“Assume $x \in X$ and $x \notin \bar{A}$."), and drawing logical conclusions (“ $C_{X}(\varnothing)=X$ and $X$ is open, so $\emptyset$ is closed.").

Checking occurred anytime Stacey attempted to verify the truth of a claim. Unlike the checking documented in Carlson and Bloom (2005), which consisted of a comprehensive evaluation of the final answer and solution to a problem, Stacey's checking took place throughout her proving process. Additionally, while Carlson and Bloom identified "internal" 
checking processes, by means of which their research subjects verified their own work, we included "external" checking actions (i.e., asking questions) in this code as well. Stacey sometimes appeared to be unsure of the correct implementation of notation and sentence structure necessary for the construction of a rigorous proof using topological and set theoretical concepts. For example, in the excerpt of coded transcript presented in Appendix A, after describing an outline for a proof, Stacey asked "Can you say that points are disjoint, or does it have to be sets?" For a question to be coded as checking, it must be a check for language and notation usage or a confirmation of logic or content knowledge (e.g., "We want to say that $\mathrm{X}$ is closed, so that can't fit the definition, 'cause we need X to be open for the null set to be closed. Or is the null set like a special case with that?"). These types of questions are distinct from those asked in the orienting phase, as they are not asked for the sake of understanding the problem but rather to ensure the correctness of the solution.

\section{Results}

Across group study sessions, we observed several instances of Stacey producing visual representations in the form of diagrams that represented a prominent definition in the problem statement. Stacey used these diagrams as tools in her search for the key idea of the proof in each session. Stacey also spent time in each of the four phases of the Multidimensional ProblemSolving Framework (orienting, planning, executing, and checking). However, the behaviors in which she engaged during her time in each phase differed somewhat from the specific behaviors observed in the experts in Carlson and Bloom's study, and the order in which she passed through these phases was inconsistent. 


\section{Stacey's Diagrams and Their Role in the Identification of Key Ideas}

During Sessions 1 and 3, Stacey produced diagrams when prompted by the facilitator, but it wasn't until Session 5 that Stacey spontaneously produced a diagram for the first time. During the Prove task, Stacey spontaneously produced diagrams in Sessions 5, 7, and 9. Stacey did not produce a diagram during the Prove task in Session 6, and in Session 8, no Prove task was given due to time constraints. The diagrams Stacey drew in Sessions 5 and 7 resulted in quick recognition of key ideas and subsequently led to formal proof production. While the diagrams produced in Session 9 did not result in immediate recognition of key ideas or the ultimate production of a proof, Stacey's focused use of one diagram to investigate the effects of individual properties led to significant evolution of her thinking. We argue that this exploration of properties was a concerted effort in search of the key idea.

In Session 5, Stacey and another student, Tom, were presented with the following statement:

Prove: A subset A of a topological space $(X, \mathcal{T})$ is said to be dense in $\mathrm{X}$ if $\bar{A}=X$.

Prove that if for each open set $O \in \mathcal{T}$ we have $A \cap O \neq \emptyset$, then $\mathrm{A}$ is dense in $\mathrm{X}$.

(Note: the notation $\overline{\mathrm{A}}$ indicates the topological closure of $\mathrm{A}$ in $\mathrm{X}$.)
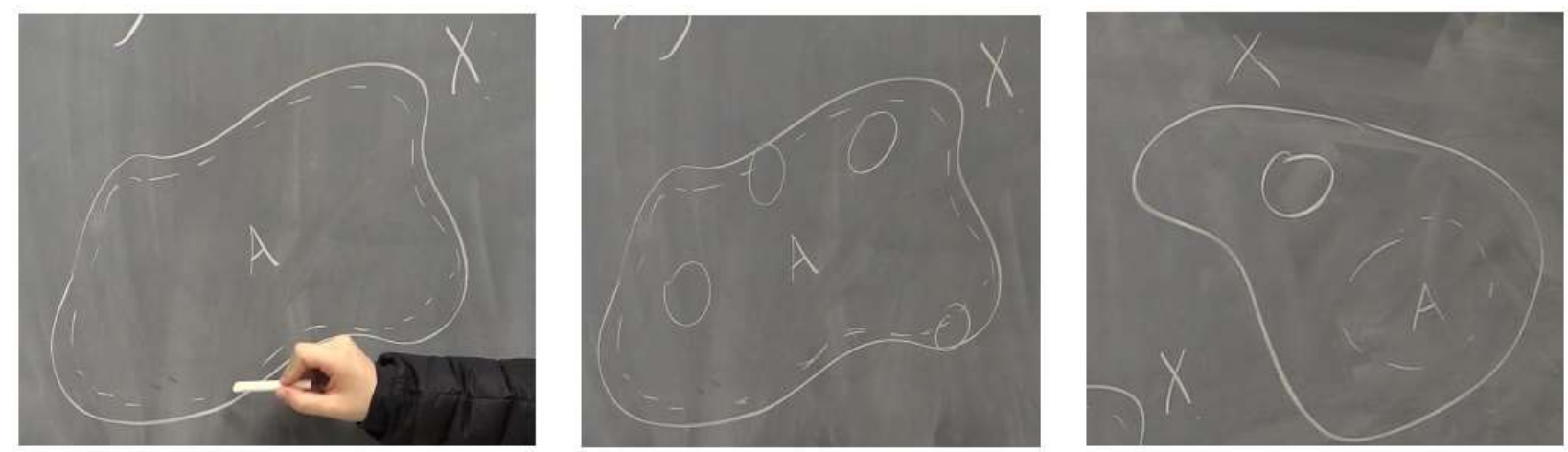

Figure 1(a-c): Stacey's diagrams from Session 5. 
Both students silently read the above prompt to themselves, and Stacey drew the diagram in Figure 1a. She explained:

I can't really show it with a picture because I can't draw, like, a dashed line over a straight line, or like, a solid line, but we have $X$ [2 sec pause] on the outside [2 sec pause] and then we have the set $A$ [2 sec pause], which is represented by the dashed, which I wish I could get closer to this [pointing to the border of $X$ ], but I can't. So if we had the closure of $A$, then it would just be the same as that solid [1 sec pause] line [tracing the border of $X$ with her hand]. So then if you take [2 sec pause] any open set [drawing circles on her diagram, Figure 1b] [2 sec pause] anywhere, there has to be some kind of intersection [2 sec pause] with $A$. So if it wasn't, like if you take [2 sec pause], if the intersection could be closed, er, could be, not closed, um, the empty set - [draws the diagram in Figure 1c] you've got $X$ here, and $A$ here, and you could have an open set here, and their intersection would be the empty set. [code: recognizes key idea] But then this closure wouldn't be equal to $X$. [7 sec pause] I get it conceptually I think, but I'm not sure how to prove it.

Stacey's inflection changed noticeably when she began, "So if it wasn't..." as her explanation shifted from a simple description of the problem to her consideration of a hypothetical scenario in which there exists an open set $O$ whose intersection with $A$ is empty. The pacing of her words also changed from a more gradual description with frequent pausing to a more continuous flow of ideas as she elaborated on the significance of this hypothetical situation, suggesting a shift in her thinking. Stacey's recognition of the resulting contradiction and concurrent drawing to represent it triggered her to consider how to translate this idea into a formal proof. It is not 
Stacey's knowledge of how this idea will translate into a proof, but her assessment that it should that makes this a key idea for Stacey and for the proof she eventually produced.

Stacey was asked to prove the following statement in Session 7:

Prove: Let $(X, \mathcal{T})$ be a topological space. A separation of $X$ is a pair $U, V$ of disjoint open subsets of $X$ whose union is $X . X$ is connected if no separation of $X$ exists. If the sets $C, D$ form a separation of $X$ and if $Y$ is a connected subspace of $X$, then either $Y \subseteq C$ or $Y \subseteq D$.
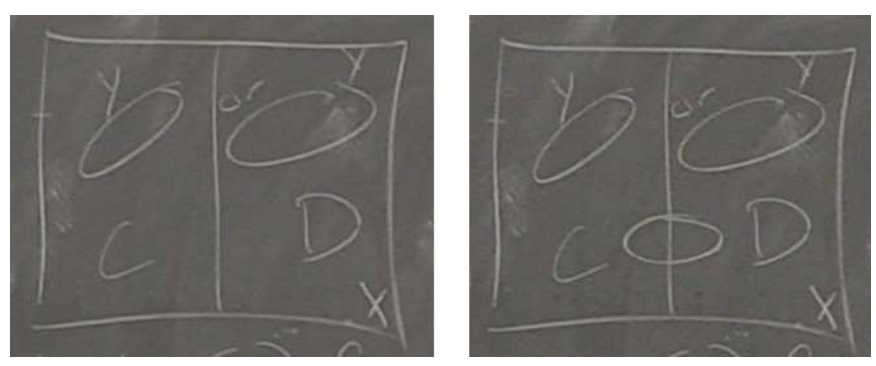

Figure 2(a-b): Stacey's diagrams from Session 7.

When presenting this problem, the facilitator neglected to include in the definition of separation that the sets $U$ and $V$ forming the separation must be nonempty. This error did not appear to affect Stacey's reasoning.

Stacey drew the diagram in Figure 2a and wrote a few words to begin her proof, but she stopped herself to explain:

If you have $X$, the ambient space, and then you have the sets $C$ and $D$, they form a separation, so that means that they're disjoint, so they don't have any of the same elements, and that their union is $X$, so that is satisfied for this. And then if $Y$ is connected, which means it's not in these sets that are disjoint whose union is $Y$, it's just one cohesive set, then it has to be either in $C$ [1 sec pause] or [1 sec 
pause] in $D$ [2 sec pause] It can't be in both, because if it was like that [draws the subset in Figure 2b], it would be disjoint. [code: recognizes key idea]

Note that while Stacey concluded this excerpt with the word disjoint, we believe she meant to say disconnected, as she had just identified the key idea and her proof followed appropriately. As with Stacey's explanation of her diagram in Session 5, her vocal cadence increased when she began to describe the hypothetical scenario at the end "Because if it was like that..." Stacey's diagram production and subsequent analysis seemed to lead her to recognize the key idea, which in turn led Stacey to begin writing a formal proof right away, and her proof (by way of contradiction) focused on this idea of "What if $Y$ was not a subset of $C$ or $D$ ?"

Stacey produced two distinct diagrams in Session 9. The prompt in Session 9 was the following:

Prove: If $Y$ is a compact subspace of the Hausdorff space $X$, and $x_{0}$ is a point of $X$ that is not in $Y$, then there exist disjoint open sets $U$ and $V$ containing $x_{0}$ and $Y$, respectively.

Referencing her textbook, Stacey drew the first diagram (Figure 3) while the prompt was still being written on the board.

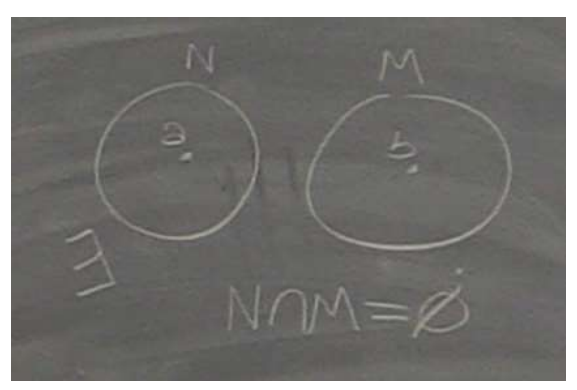

Figure 3: Stacey's diagram representing the Hausdorff condition in Session 9.

She explained very briefly, "Just reminding myself what Hausdorff is." In contrast to the diagrams produced in Sessions 5 and 7, however, Stacey did not talk much about this one, nor 
did she interact with it after its initial production. Rather, she translated some of the information from the problem into notation (Figure 4 ) and briefly noted aloud a few facts like " $Y$ is Hausdorff" and " $Y$ is closed" before drawing a second diagram (Figure 5):

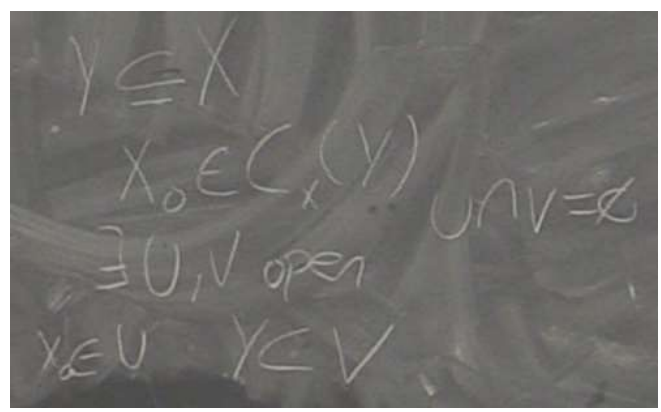

Figure 4: Stacey's notation from the Prove task in Session 9.

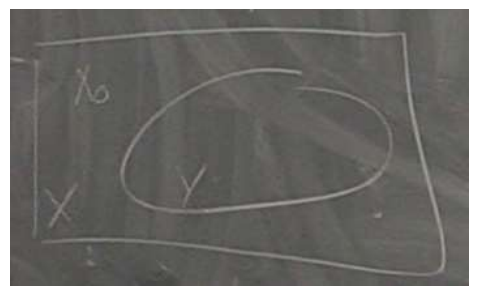

Figure 5: Stacey's diagram representing the problem scenario in Session 9.

Unlike the diagrams Stacey produced in Sessions 5 and 7, the diagram in Figure 5 saw heavy use. In Sessions 5 and 7, Stacey was very quick to recognize significant properties of her diagram which she began to translate into proofs shortly after drawing the diagram. While Stacey completed full proofs (with some assistance) in both of Sessions 5 and 7, she never began the process of writing a formal proof in Session 9, instead spending the entire time in exploration which included several modifications to the diagram in Figure 5.

This is not to say that Stacey made no progress during Session 9. On the contrary, Stacey proposed several ideas and expressed astute observations and concerns, which she represented using her diagram. Each idea focused on one of the properties given in (or resulting from) the conjecture. Stacey first recognized that $Y$ was closed (as a compact subspace of a Hausdorff space), then set $U$ equal to the complement of $Y$ and suggested trying to find another open set $V$ 
containing $Y$. Upon recognizing that such sets $U$ and $V$ may not be disjoint, Stacey abandoned this idea. She then focused on the compactness of $Y$, which she initially said meant that "there is a finite number of open sets that can cover $Y$ " (Figure 6, top right). Stacey began to consider finding some finite collection of open sets to cover $Y$ and then taking the union of that collection, but she was unsuccessful. She also considered applying the Hausdorff property by taking a point $y_{0}$ in $Y$ and choosing disjoint open sets containing $y_{0}$ and $x_{0}$, respectively, but she expressed concern that the open set containing $x_{0}$, chosen to be $U$, might ultimately intersect the desired open set $V$ containing all of $Y$ (Figure 6, bottom right). Though Stacey never began to write a formal proof of the statement given in Session 9, her way of thinking about this problem evolved considerably during the time she spent working toward one.
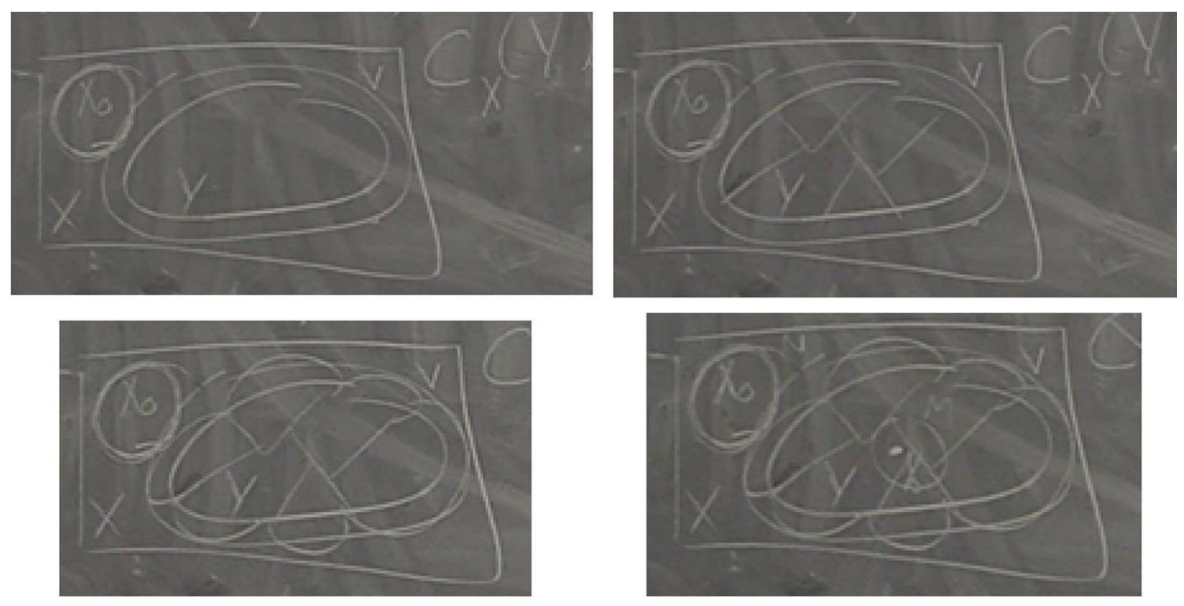

Figure 6: The evolution of Stacey's diagram in Session 9.

During Session 9, Stacey never identified the key idea of the proof, and she never began to write a formal proof. Much of Stacey's time in Session 9 was spent investigating the roles and potential advantages and pitfalls presented by individual properties of the mathematical objects involved in this problem. Stacey used her diagram extensively to explore various ideas related to her proof, but each idea centered around only one of the properties given in the problem statement, e.g., the Hausdorff property or compactness. For the successful completion of this 
proof, one must coordinate several different properties and see how they work together to imply the conclusion of the conjecture.

We emphasize here that Stacey never attempted to begin writing a proof in Session 9. On the contrary, she spent the entire duration of this session informally trying to determine the conceptual underpinnings of the problem and convincing herself why the statement is true. Rather than trying to establish the procedural idea of the proof by working formally with notation and definitions, Stacey spent her time trying to figure out a heuristic idea that she could then translate into a proof - the key idea. We argue that, although Stacey never arrived at the key idea of the proof in Session 9 like she did in Sessions 5 and 7, she made a dedicated effort to identify the key idea, and that effort was strongly linked to the diagram that Stacey produced at the beginning of her work in this session.

\section{Stacey's Progress Through the Phases of the Multidimensional Problem-Solving}

\section{Framework}

In each session, Stacey showed evidence of engaging in each of the four phases of the problem-solving cycle described by Carlson and Bloom (2005), provided that she was able to obtain a complete proof. During her time in the planning phase, Stacey sometimes engaged in the conjecture-imagine-evaluate sub-cycle but did so through interactions with her diagrams.

Furthermore, during the checking phase, Stacey demonstrated behaviors not described by Carlson and Bloom. The order in which she passed through the phases differed from those of the experts in Carlson and Bloom's study as well.

A key feature of Stacey's time in the orienting phase during Sessions 5, 7, and 9 was the production of a diagram to represent the problem scenario or a definition relevant to the problem. The problems presented in Sessions 5 and 7 introduced terminology that was new for Stacey 
(dense in Session 5 and separation in Session 7); in each of these sessions, her diagram production focused on these definitions. No new definitions were introduced in Session 9; nevertheless, Stacey drew a diagram to represent the problem spatially, and throughout her work, she used this diagram to explore the various definitions that were involved (Hausdorff, compact, closed) in the proof. She also began Session 9 by drawing a diagram to "[remind herself] what Hausdorff is," but she did not work on this diagram during her proving process.

Stacey appeared to use her diagrams during her engagement in the conjecture-imagineevaluate sub-cycle of the planning phase. For example, in Session 5, Stacey initially represented the concept of a dense subset in a diagram, and she convinced herself that the statement of the problem made sense by drawing a few open sets and seeing that they intersected with her dense subset. She then conjectured about what would happen if the problem statement wasn't true: If I could draw an open set that didn't intersect with my subset, what would that mean? Rather than internally imagining the consequences of this idea, Stacey created a visual representation (Figure 1c) to investigate her conjecture and appraise its utility. Stacey exhibited similar behavior in Session 7 when she considered what would happen if a connected subset $Y$ intersected both parts of a separation. This behavior was marked in each case by the use of "What if?" language: "So if it wasn't..." in Session 5, and "Because if it was like that..." in Session 7. Furthermore, this language was accompanied in each case by an act of drawing the conjectured situation, from which Stacey explained the implications of that scenario.

In Session 9, Stacey spent a significant amount of time in the planning phase and never moved past it into the executing phase. Stacey's planning in Session 9 consisted of several iterations of conjecture-imagine-evaluate, each of which terminated when Stacey identified a conceptual problem with her conjecture. For example, when Stacey recognized that $Y$ was a 
compact subspace of a Hausdorff space, she correctly identified it as a closed set, and she conjectured that the open set she needed containing $x_{0}$ might be $U=C_{X}(Y)$. However, she then identified that this would require that $V=Y$ be the other open set named in the problem, causing her to reject her hypothesis and identify another conjecture. During each iteration of the subcycle, Stacey's consultation or modification her diagram led to the rejection of her conjecture.

In Sessions 5 and 7, Stacey's time in the planning phase was dominated by her conjecture-imagine-evaluate activities. In each case, Stacey quickly recognized that If the conclusion of the statement doesn't hold, then the hypothesis can't be true, and she decided to approach each proof by way of contradiction. This led Stacey to transition into the executing phase and begin to construct her proofs. However, in each case, Stacey occasionally returned to the planning phase while writing her proof to decide on the best way to accomplish a particular step in her proof. For Stacey's proof in Session 7, she assumed that her connected subspace $Y$ intersected both parts $C$ and $D$ of the separation of $X$, and she began by considering elements $x$ and $y$ in $C$ and $D$, respectively. However, after working for a few minutes, Stacey asked "Should I have picked subsets of $Y$ to start with? That would have made this a lot simpler," signaling a return to the planning phase as she began to assess the effectiveness of a different strategy.

In Carlson and Bloom's study, the experts "spontaneously assessed the correctness of their computations and results" (Carlson \& Bloom, 2005, p. 63) in the checking phase. While Stacey engaged in this behavior, she also conducted extensive external checking, i.e., conferring with other students present in a given session or with the facilitator to verify her reasoning. In further contrast to the experts in Carlson and Bloom, much of this external checking pertained to mathematical language and notation usage. In Session 7, Stacey wrote "Let $Y \subseteq C$ and $Y \subseteq D$," and explained "If we do it by contradiction, and we'd say that there's intersection with both of 
them, and then you can show that $Y$ can't be connected. Would that be OK?" Stacey's expression of her idea showed that there was a mismatch between her intention and her notation. Recall that $C$ and $D$ form a separation of $X$ and therefore are disjoint. What Stacey's notation indicated isn't possible in this context, but her intention to assume that $Y \cap C \neq \emptyset$ and $Y \cap D \neq \emptyset$ is permissible. Stacey performed a significant amount of checking in this way throughout every session, first expressing an idea and then requesting feedback regarding the appropriate way to use mathematical language and notation to formalize it.

Stacey's actions during her time in the executing phase of the framework were largely unremarkable. What is worth mentioning is the interplay of planning, executing, and checking that took place during Stacey's proof writing process. Once Stacey entered the executing phase and began to assemble a formal proof, her transitions among these three phases became nonlinear. We present an excerpt from a coded transcript in Appendix A. She returned to the planning phase from the executing phase throughout writing a proof when she got stuck on a particular step in her proof, for example in Session 7 when Stacey struggled to find the pair of disjoint open sets that would produce her desired contradiction as described earlier in this section. She sometimes transitioned from planning into (external) checking to determine whether her plan was viable prior to implementation. If Stacey returned directly from planning to executing, she typically then moved quickly into checking after one or two individual steps in her proof, either to confirm logic or to verify language and notation.

\section{Discussion}

\section{Stacey's Diagrams as an Aid to Recognizing Key Ideas}

Stacey's use of her diagrams in the course of the conjecture-imagine-evaluate sub-cycle of the planning phase led her to the recognition of the key idea of each proof, which she then 
transformed into a formal proof. Of the three sessions presented, Session 9 was the only one in which Stacey did not produce a proof, but her repeated reference to her diagram allowed her to reject a sequence of unproductive conjectures. In each of Sessions 5 and 7, Stacey's proofs were preceded by the construction of a diagram. Each diagram represented a definition that was introduced in the statement Stacey was asked to prove. Avoiding what Presmeg called "one-case concreteness," (1986, p. 301) each diagram merely captured the essence of the definition, maintaining abstractness and generality.

Mason (1992) and Booth and Thomas (2000) argued that possession of a diagram did not guarantee its usefulness, but that students must actively work on their diagrams in order for them to be useful. Stacey's diagrams in Sessions 5, 7, and 9 were used primarily for experimentation and exploration. During these sessions, Stacey engaged in conjecturing during the planning phase, and she used her diagrams to model the conjectured scenarios and evaluate the usefulness of each one. In Sessions 5 and 7, she arrived quickly at a useful piece of information: If there exists an open set that does not intersect $A$, then the closure of $A$ will not be equal to $X$ in Session 5, and If $Y$ intersects both $C$ and $D$, then there will exist a separation of $Y$ in Session 7. The recognition of these ideas cued Stacey to approach each proof using the method of proof by contradiction. Stacey's success in these sessions hinged not on the fact that she drew a diagram, but on the fact that she drew a diagram with the intention of extracting useful information from it (Stylianou, 2002). The construction of a formal proof necessitates the use of definitions.

However, as Moore (1994) suggested, Stacey's initial reading of the definitions in Sessions 5 and 7 prompted her to construct an image, informing her understanding of each concept, which then allowed her to make more efficient use of the definitions in her subsequent proofs. 
This experimentation behavior resembled the conjecture-imagine-evaluate sub-cycle described by Carlson and Bloom (2005). Indeed, Stacey's experimentation included the investigation of conjectures and the acknowledgment of the consequences of such conjectures. However, while the conjecture-imagine-evaluate sub-cycle occurred internally for the subjects of Carlson and Bloom's study (either through silent consideration or verbal description), Stacey's behavior was manifested externally, with her imagination of the consequences of her conjectures being accompanied by modifications to her diagrams. This may result from a difference in experience and mathematical maturity between Carlson and Bloom's subjects and Stacey. The conjecture-imagine-evaluate cycle may occur internally for expert mathematicians, but is this necessarily so for novices? Furthermore, for the experts, this cycle resulted in a procedural approach to solving a problem which could subsequently be executed. For Stacey, this cycle yielded conceptual ideas about a proof. While Stacey seemed to acknowledge that there should be some sort of bridge between the conceptual idea and the procedural idea, crossing that bridge was not automatic. She may have recognized that, if a set $Y$ intersects both parts of a separation, then $Y$ must not be connected, but playing out the steps in the proof appeared to only take place externally when Stacey moved into the executing phase.

Raman's (2003) definition of key idea as "an heuristic idea which one can map to a formal proof with appropriate sense of rigor" (p. 323) describes this behavior perfectly. Stacey's utterance in Session 5 that "I get it conceptually I think" pointed toward a personal understanding of the situation; Stacey had ascertained (Harel \& Sowder, 1998) why the statement is true (the heuristic idea). Though she then claimed to be unsure how to prove the statement, Stacey expressed her intention to prove it by way of contradiction soon after as a means of translating her heuristic idea into a formal proof. Her behavior in Session 7 followed 
the same pattern: once Stacey had produced her diagram, she explained what would happen if $Y$ intersected both sets $C$ and $D$ and immediately began to write a proof by way of contradiction.

In contrast, Stacey never arrived at the key idea of the proof for the statement given in Session 9. Rather, she spent the entire session in exploration, investigating the implications of the individual properties required by the hypothesis of the statement. Stacey's explorations, however, suggest a concerted effort to discover the key idea of the proof. Recall that, while Stacey showed no evidence of having recognized the key idea, she also made no attempt to begin writing a proof. Furthermore, Stacey expressed concern over her inability to find open sets $U$ and $V$ that would be disjoint. In its own way, this might be interpreted as a "What if?" question, similar to those Stacey asked in Sessions 5 and 7. What if the open sets $U$ and $V$ did intersect?

We make two observations here about the differences between Sessions 5 and 7 and Session 9. Perhaps the most apparent of these is Stacey's chosen proof strategy in Sessions 5 and 7 , namely, proof by contradiction. In the authors' opinion, proof by contradiction is the simplest and perhaps the most obvious way to prove the statements given in Sessions 5 and 7. The problem given in Session 9, however, yields most easily to a direct proof. This raises potential questions. Is it simply easier, in general, to recognize the key idea of a proof by contradiction? Are diagrams more useful for key idea recognition when the method of proof is the derivation of a contradiction than they are when the method is direct proof?

Our second observation regards the level of complexity in the proof tasks. The problems in Sessions 5 and 7 both introduced new definitions, but the proof tasks, themselves, were relatively uncomplicated. In Session 9, Stacey appeared to have little difficulty identifying the implications of individual topological properties, such as the Hausdorff property, compactness, or the closedness of sets. Her difficulty, it seems, is in the coordination of two or more properties 
simultaneously. The proof of this claim relies on the interplay of both compactness and the Hausdorff property: the Hausdorff property allows for the identification of pairwise disjoint open sets, and the finite collection of open sets containing $x_{0}$ guaranteed by compactness means that the intersection of such a collection will also be open. Ignoring either of these properties makes it impossible to complete this proof, so the ability to consider the implications of both properties simultaneously is essential for success in this problem. It should be noted that, on the basis of our evidence, we cannot claim that Stacey would not have reached this conclusion if she were given more time. It does seem, however, that while Stacey understood each individual component of this problem, she struggled to make connections between them. In Session 5, Stacey remarked, "I don't know why, but I'm having trouble like, reconciling it between having $A$ and $X$ and open sets and closures... being able to put all those in the right order," pointing to this difficulty in coordination that appears to affect Stacey's success. Stacey was able, with support, to produce a proof during Session 5, but the escalation in complexity in Session 9 may have pushed the difficulty past a certain threshold for her. Though she may have been able to complete a proof with more time, there is a noticeable difference in her ability to cope with such a difference in complexity.

\section{Stacey's Behavior in the Phases of the Multidimensional Problem-Solving Framework}

Stacey displayed many of the behaviors that were exhibited by the experts in Carlson and Bloom's (2005) study, but she also displayed several others. Like Carlson and Bloom's subjects, Stacey was adept at producing visual representations to orient herself to problems. Using these diagrams to consider the consequences of various properties given in the problems and to ask "What if?" questions, Stacey attempted to determine the key idea of the proof, allowing her to transition into the planning phase. However, Stacey's planning seemed to take place more "on- 
the-fly": she would decide upon a step in her procedure, execute that step, and check it before deciding on the next step. As a consequence, Stacey's time in the executing and checking phases occurred in smaller cycles corresponding to individual steps of the resulting proof, rather than taking place in one complete proving cycle.

Stacey's checking behaviors differed from those observed by Carlson and Bloom. While she did appear to self-monitor to a degree, much of her checking was conducted externally by means of conferring with a partner or with the facilitator. Furthermore, her checking was not restricted to monitoring of conceptual accuracy, but also linguistic and notational correctness. Stacey asked many questions of the facilitator and of her partners throughout each of the sessions requesting clarification on concepts. However, she also frequently asked questions to ensure that she was using correct mathematical notation and that her word choice and syntax were appropriate to express the ideas she wanted to convey in her proofs.

Such behavior was unlikely to be seen in the subjects of Carlson and Bloom's study because these subjects were experienced mathematicians. Although the problems, themselves, were probably unfamiliar to Carlson and Bloom's subjects, it is likely that they had a significant amount of experience working with the concepts and the notation as well as mathematics in general. For her part, Stacey lacked all of these advantages. Some of the problems used during the course of this study introduced topics that Stacey had not encountered previously. Regarding the topics Stacey had already seen, many of them were covered for the first time recently in class and had perhaps not had a chance to solidify in her mind. In a more general sense, Stacey was a novice mathematician at the time of this study, and her fluency with logic, language, and notation was still developing. Stacey's mathematical inexperience is likely at the heart of the difference in planning as well, as Stacey's ability to think several steps into a problem was 
probably hindered by the amount of cognitive engagement required for her to complete a single step in her head. She had enough experience to recognize the usefulness of broad strategies like proof by contradiction, but the more detailed work required to produce the specific proofs necessary may have required too much mental processing power to be within Stacey's abilities at this point in her mathematical career.

\section{Conclusions and Implications for Teaching}

Our case study of Stacey and her use of visual representations when attempting to write proofs in the context of introductory topology revealed a tendency to use diagrams to help identify the key idea of a proof. Many authors have suggested that visual representations are of little use unless students actively engage with them (Booth \& Thomas, 2000; Carlson \& Bloom, 2005; Mason, 1992; Stylianou, 2002; R. Zazkis et al., 1996); this study gives some insight into the (possibly implicit) goals some students may seek to accomplish when they inspect their diagrams.

It is worth recalling that this study investigated a student's behaviors in the context of proving and not problem solving. This focus on generality and logical precision of language and notation may elicit from students a different way of thinking than the specificity and computations required in solving a specific, more computational problem. Further, this case study focused solely on the behaviors of Stacey, as she was the most active of our study's participants. Though we were able to identify this behavior in Stacey, the prevalence of this particular behavior among students in general is open for study, as is the exploration of other uses of visual representations demonstrated by students writing proofs in introductory topology.

With regard to the Multidimensional Problem-Solving Framework (Carlson \& Bloom, 2005), we do not find Stacey's checking behaviors to be surprising. As a relatively inexperienced 
mathematician and a newcomer to the field of topology, Stacey's insecurity regarding her language and notation are understandable. Despite the challenges with formality, Stacey's time spent working on her diagrams in the orientating phase often gave her the informal underpinnings of what would later become a formal proof, with the appropriate guidance. It is also unsurprising to us that Stacey's plans for a proof often came in a piecemeal fashion rather than all at once. Again, due to Stacey's relative inexperience with proving and with topology, her attention and mental processing power may have been divided among those features of mathematics that more experienced provers might have relegated to procedural memory, such as notation, word choice, and syntax. Even visualization may take place completely internally for more experienced mathematicians, as some of these images may have been committed to memory through prior use. The amount of Stacey's focused attention required by each of these processes, along with the actual conceptual reasoning involved, likely created a substantial barrier to her ability to think several steps into a multi-step proof.

It has been suggested previously that students do not spontaneously attempt to make sense of new or difficult content, but that informal generative learning activities (e.g., drawing a picture) can require students to actively make connections and engage with content (Fiorella \& Mayer, 2016). We found evidence in 6 of the 9 distinct sessions to suggest that the use of visualization and visual representations may support students' sense-making activities when writing proofs. Furthermore, the results of this study indicate that the construction of a visual representation of a key definition or concept in the statement of a theorem can lead to the recognition of the key idea of the proof. Though students are likely to require guidance using formal mathematical notation and language, the results of this study support the effectiveness of students performing generative learning activities such as drawing a picture or explaining 
problems or concepts in their own words as aids in proving. These strategies can help students to informally "get the idea" and allow them to establish a trajectory that their formal mathematics will then follow. Particularly in introductory courses, many mathematical ideas follow from a sort of "common sense," but that common sense is often obscured by highly technical and specialized mathematical language and notation. Giving students the tools to see the "common sense" reason for the truth of a conjecture may allow students to discuss and reason about advanced mathematics in an informal way, making complicated ideas more accessible and improving student engagement. 


\title{
PAPER 2: STRUCTURAL EXAMPLES: REPRESENTING \\ ABSTRACTNESS IN TOPOLOGY
}

\begin{abstract}
Proof writing is considered one of the most important aspects of advanced mathematics. It is known that expert mathematicians use examples and visual representations as part of their informal mathematics when working toward proof construction. However, the ways undergraduates understand and work toward creating proofs is an open area of investigation, particularly in advanced mathematics. Furthermore, research on students' learning and understanding in topology is largely unexplored. We present a case study of an undergraduate taking a first course in general topology and her informal reasoning which led her to produce formal proofs and counterexamples. In particular, we observed that she rarely produced "traditional" examples, but in many instances, she reasoned about more abstract "structural" examples, which seemed to aid in her proving processes. We argue that these structural examples may be useful for students writing proofs in topology, particularly when students may not have access to specific examples.
\end{abstract}

\section{Introduction}

Examples are thought to be essential to the development of mathematical understanding (Alcock, 2004; Alcock \& Inglis, 2008; Alcock \& Weber, 2010; Bills \& Tall, 1998; Mason \& Watson, 2008; Tall, 2008; Watson \& Mason, 2005; Weber \& Alcock, 2009). However, courses in advanced mathematics often focus on the presentation of definitions and theorems and the construction of proofs rather than on the generation and exploration of examples. In particular, topology is one of the most common proof-based courses in the American university system (Fukawa-Connelly \& Newton, 2014), yet little research on student thinking and learning has 
been conducted in the context of undergraduate topology, and even less of that research has focused on students' exemplification.

Cheshire (2017) studied undergraduates' development of axiomatic thinking in topology, exploring the challenges these students encountered while transitioning into the axiomatic system of topology. With regard to instantiation, Cheshire reported on students' uses of various conceptions of the notions of open sets and continuous functions. Berger and Stewart (2018) reported on students' development of schema for a topology generated by a basis, showing that even at the end of a semester-long course in general topology, most of their students' schema for this topic were still in the beginning stages of development. Gallagher and Engelke Infante (2019) presented a case study analyzing the role visual representations played in the proving activities of an undergraduate student in introductory topology and how those representations helped her identify the key ideas in her proofs. None of these studies, however, takes the process of exemplification as its central focus.

What constitutes an example? Fukawa-Connelly and Newton (2014) emphasize a dichotomy between examples of processes (like the division algorithm) and examples of concepts (like groups and fields). Our focus in this paper will be to identify and describe examples of concepts in topology and the ways those examples are used in proof construction. Watson and Mason (2005) defined an example as "any mathematical object from which it is expected to generalize" (p. 3); following this same theme, Alcock and Weber (2010) defined an example of a concept as "a mathematical object satisfying the definition of some concept" (p. 2).

In a study of when and how university faculty teaching proof-based, upper-division mathematics courses used examples when presenting proofs, Mills (2014) described an example in the following way: 
$[I] t$ must be specific and concrete as opposed to general and abstract. Specificity is a mathematical requirement; the object must represent a particular element of a larger class. Concreteness implies that students at this level must be able to either compute with it or investigate properties of the mathematical object. Thus, concreteness is concerned with the accessibility of the mathematical object to the learner. Therefore, I will use the following definition: An example is a specific, concrete representative of a class of mathematical objects, where the class is defined by a set of criteria. (p.107)

Along the lines of Watson and Mason's (2005) definition, however, we propose that specificity is not necessarily a required property of an example, only what Mills called concreteness. Specificity may be essential for examples of processes, but abstract entities, such as the "blobs" Mills described (2014, p. 107), can be used to develop the kind of general understanding that is required for a proof, despite their lack of specificity. In our data, we present a case study of a student, Stacey, who produced what Mills (2014) called generic diagrams. Mills chose not to include diagrams of this kind in her classification of examples, as they lack the necessary specificity to represent a particular element of a class. However, we will demonstrate that Stacey was able to use such diagrams to investigate the properties of mathematical objects for the purposes of proof and counterexample construction. Thus, we define an example to be any mathematical object satisfying the definition of some concept, from which it is expected to generalize or to investigate the properties of the object. Due to Stacey's uses of her diagrams to build her general understanding of concepts and to investigate their properties, we refer to her generic diagrams as structural examples. 
Structural examples represent a form of structural reasoning (Dorier \& Sierpinska, 2001; Harel \& Soto, 2017; Leron, 1983, 1985). Harel and Soto (2017) provided the following definition of structural reasoning:

Structural reasoning is a combined ability to: (a) look for structures, (b) recognize structures, (c) probe into structures, (d) act upon structures... (e) reason in terms of general structures... (f) the ability to see (be aware of) how a piece of knowledge acquired resolves a perturbation experienced... (p. 226)

This definition gave rise to a typology categorizing structural reasoning into five behaviors. Most relevant to this discussion will be the behaviors of "recognizing and operating with structure in thought... epistemological justification... [and] reasoning in term[s] of general structures" (Harel \& Soto, 2017, p. 232). The practice of epistemological justification arises out of the constructivist notion that

for any piece of knowledge $K$ possessed by an individual or community, there exists a problematic situation $S$ out of which $K$ arose. $S$, prior to the construction of $K$, is referred to as an individual's intellectual need: $S$ is the need to reach equilibrium by acquiring a new piece of knowledge. (Harel \& Soto, 2017, p. 230)

In other words, an individual's creation of a new piece of knowledge or mental object must be stimulated by a problem to which that knowledge is the answer. In this study, Stacey's intellectual need stems from her need to prove or disprove a mathematical conjecture, which requires her to generate a new (for her) mathematical object (a structural example). To resolve her intellectual need, Stacey must recognize and reason in terms of general structures as well as act upon them in order to see how the structure she has generated resolves the perturbation caused by encountering the mathematical conjecture. 


\section{Generalization Versus Abstraction}

Generalization and abstraction are both means of reducing complexity in a system by identifying components of the system's structure. These words are often used interchangeably, but we believe that there is a subtle yet important difference, particularly within the realm of mathematics learning.

Generalization is the cognitive process of reducing complexity by recognizing patterns; this may be thought of as a "bottom-up" approach to recognizing mathematical structure. To generalize is to use specific cases to identify similarities and differences among examples, resulting in the recognition of a set of governing rules through a process of comparing and contrasting (Harel \& Tall, 1991). It is not enough to simply guess at these rules; one must truly have a sense of the structure underlying those examples (Mason, Stephens, \& Watson, 2009). That is, one who is able to successfully generalize a concept has a grasp of the dimensions of possible variation, the components of an example that can be changed without removing its status as an example (Mason \& Watson, 2008). Furthermore, one must acknowledge the full extent of the range of permissible change of each such dimension, the set of viable choices for those properties that can change (Mason \& Watson, 2008). One who has successfully generalized a concept has the ability to generate many, or infinitely many, examples of the given concept, as they know exactly what can change and how much it can change. By extension, however, one must also recognize that which must remain the same; that is, what is "invariant in the midst of change" (Mason \& Watson, 2008, p. 195). In courses like calculus and geometry, this is often where students begin with proof: they search for inductive evidence of truth, that is, they convince themselves of the truth of a statement by consideration of several examples, examples which sometimes may seem very different but share a common underlying structure. 
This is useful for developing internal conviction of the truth of a statement; what Harel and Sowder (1998) call "ascertaining" and Raman (2003) calls the "heuristic idea" of the proof. However, inductive evidence and internal conviction are not enough to guarantee universal validity of a claim - infinitely many examples may exist to support a claim, but this does not preclude the existence of a counterexample.

Abstraction, on the other hand, is a cognitive process of reducing mathematical complexity by recognizing underlying structure (Gray \& Tall, 2007; Harel \& Tall, 1991). In contrast to generalization, abstraction may be thought of as a "top-down" approach to thinking about mathematical structure. Abstraction need not be the result of considering several examples; in fact, one need not ever encounter a single example. One who has successfully abstracted a concept need not be aware of the dimensions of possible variation within that concept nor their ranges of permissible change but must be acutely aware of what is invariant in the midst of change. As such, they can work with the concept itself as representing an entire class of examples because they recognize that every such example must share certain common properties. This allows them to draw conclusions about every such example without ever conjuring a specific example to think about: "Any arguments valid for the abstracted properties apply to all other instances where the abstracted properties hold, so (provided that there are other instances) the arguments are more general" (Harel \& Tall, 1991, p. 40). This is the essence of proof: we expect our students to develop the ability to draw conclusions about all mathematical objects within a class based on the essential properties of those objects without the need for recourse to specific examples. We acknowledge, however, that consultation with specific examples is often helpful for developing intuition about a concept, as specific examples allow us to work within familiar structures and to recognize the breadth of possibilities and to search for 
counterexamples. Abstraction allows one to work directly with the underlying mathematical structure, itself, without the need for examples. It is what is necessary for successful proof writing, as one can work with the structure of all examples simultaneously rather than specific cases, guaranteeing the truth of a statement in general. Additionally, abstraction may be useful for the construction of counterexamples, as a deep understanding of a concept's underlying structure may help to identify which component of that structure is being neglected or invalidated by a false statement, at which point one need only generate one specific example possessing the necessary property. Leron (1985) referred to this as using "the given constraints to search for the form of the solution-object" (p. 8).

Introductory mathematics is often taught with a "bottom-up" approach, in which specific examples are shown which possess many unnecessary properties but exhibit the required properties and structure that we want our students to abstract. As such, it is no wonder that our students should be skilled in example generation and use inductive methods of "proof" while struggling with deductive methods. However, at more advanced levels of mathematics, concepts are more often taught in a "top-down" structure, in which instruction focuses on theorems and proofs - the general structure of a concept - and less attention is given to specific examples, except when the need arises to find a counterexample to a false claim. In such "top-down" courses, we hypothesize that students will struggle more with the generation of specific examples than they might in a "bottom-up" course. Conversely, students in "top-down" courses might be more apt to produce (or attempt to produce) deductive arguments than students in "bottom-up" courses.

Topology is one such course that may be taught in a "top-down" way. Many (though certainly not all) courses in general topology begin in relatively familiar metric spaces, which 
might allow for a comfortable transition into deductive thinking by permitting students the ability to work with many familiar examples. However, one of the goals of a course in general topology is often to teach students the skill of abstraction, hence these courses are often taught in the familiar "definition-theorem-proof" style (Cheshire, 2017), and students may struggle to generate specific examples, as they may have little to no experience working in non-metrizable spaces and may have never encountered ideas such as separability or compactness, and their instructors may place less emphasis on the specific and greater value on the abstract.

With this research, we investigated the following research questions: what kinds of examples do students use to help them write proofs in topology, and how do they use them? In this paper, we describe the proving efforts of Stacey, an undergraduate in a general topology course. Throughout the course of our data collection, Stacey continued to provide evidence of her desire, and struggle, to produce specific examples of concepts in topology. Yet, despite this (or perhaps because of it), Stacey was often successful in constructing proofs and discovering counterexamples through her ability to abstract topological ideas. In the course of these activities, Stacey generated several objects, which we call structural examples. While she often did not generate specific examples, she produced structural examples which she used to reason about concepts and proofs. Though her structural examples did not lead her to generate specific examples, they did seem to be helpful to her in constructing deductive arguments. The tasks in our study were all proof-related. In cases where Stacey was proving a statement true, her structural examples seemed to provide a help in determining an appropriate start to the necessary argument. In contrast, when she was proving a statement false, it was necessary that she produce a counterexample. In those cases, she fell back to familiar examples in $\mathbb{R}$ or $\mathbb{R}^{2}$, but her structural examples provided insight into her counterexample choices. We argue that, although 
Stacey's structural examples were useful in her quest for successful deductive arguments, they did not seem to help her when she needed to produce specific instances of new concepts. Nevertheless, they provide evidence that Stacey was able to abstract mathematical concepts without necessarily having access to specific examples of those concepts. Moreover, Stacey's structural examples did seem to support her counterexample generation by helping her identify which part of a conjecture would be problematic.

\section{Theoretical Perspective}

In this paper, we discuss instances of the generation of abstract examples in response to proof-related prompts in general topology. The theory of constructivism proposes that knowledge and understanding are created in response to an intellectual need; in other words, learning is stimulated by the need to solve a problem that cannot be solved with the knowledge one currently possesses (Harel \& Soto, 2017). According to constructivism, knowledge is constructed by the learner. Teachers can expose students to information, but this does not guarantee that the student will learn; it is up to the student to internalize and organize the information in a way that makes sense to them, and to reorganize their understanding as needed to accommodate new information (Piaget, 1985).

A commonly held value among mathematicians is that mathematical truths can be deduced a priori from certain assumptions and definitions (Dawkins \& Weber, 2016). Similarly, examples may be constructed based on some kind of prior understanding, either of concepts or of example generation techniques. The skill of example generation is invaluable for expert mathematicians (Alcock, 2004; Alcock \& Inglis, 2008), and there are frequent calls for the improvement of methods for teaching students how to generate examples (Mason \& Watson, 2008; Sinclair et al., 2011; R. Zazkis \& Leikin, 2008). The ability of proofs and examples to be 
generated from prior knowledge, rather than simply repeated, is consistent with Piaget's notion of constructivism (1985). The ability of a student to construct knowledge for themselves is essential to the learning of mathematics, and is often implicitly assumed by teachers, as many of us hope that our students will develop the ability to think through challenging new problems rather than simply repeat routine procedures demonstrated in class. The ability to construct knowledge and understanding is particularly essential to proof construction, which often requires students to not only recognize or develop the steps in a proof, but also to forge the logical connections that justify those steps. Furthermore, students and experts alike often find themselves generating their own examples to reason through proofs that use unfamiliar concepts. An individual's personal example space is populated by the examples to which that individual has access as well as any methods for example generation the individual possesses (Watson \& Mason, 2005). Far from being simply a haphazard collection of examples, every individual's personal example space has structure, which can be influenced by the experiences and values of the individual (Sinclair et al., 2011; R. Zazkis \& Leikin, 2007, 2008). Sinclair et al. (2011) described four dimensions along which the personal example space may be structured: population, generativity, connectedness, and generality. While population refers to the number of examples known by an individual, generativity refers to the possibility of producing more examples using known rules for example construction. The ability to generate new examples hinges on the individual's understanding of the dimensions of possible variation within a particular class of examples and the corresponding ranges of permissible change (Mason \& Watson, 2008). These describe the properties of an example which can be changed, and the extent to which they can be changed, without robbing the example of its "examplehood." Even after the presentation of several examples, students may still struggle to generate additional 
examples due to lack of experience in identifying the dimensions of possible variation within a class of examples (Marton \& Tsui, 2004). Connectedness describes an individual's awareness, or lack thereof, of relationships among particular examples; that is, any similarities or differences among examples such as their properties or purposes. The generality of a particular example within the space is the extent to which that example is understood to be representative of a larger class of examples. This notion correlates directly with Mason and Pimm's (1984) concept of generic examples, those examples which are seen by the individual to be representative of an entire class of examples.

Examples can give insight into and help structure an individual's concept image (Bills \& Tall, 1998). An individual's example space for a particular topic is a subset of their concept image. One's concept image is "the total cognitive structure associated with the concept, which includes all the mental pictures and associated properties and processes" (Tall \& Vinner, 1981, p. 152). As students work with a concept, their concept image is likely to change in accordance with their experiences. One's concept image need not always be coherent, but they need not be aware of any inconsistencies. While the individual's concept image may be complex, the entire image need not be evoked at once; it is only when contradictory pieces of the concept image are evoked simultaneously that a problem may be detected. The formal concept definition (Tall \& Vinner, 1981) plays a role in shaping the concept image, though the concept image need not be an accurate reflection of the formal definition. In spite of this, students often leverage their concept image, rather than the formal concept definition, when working with concepts in new contexts such as proof writing (Bills \& Tall, 1998; Moore, 1994).

The ability to reason structurally (Harel \& Soto, 2017) is essential in advanced mathematics. Structural reasoning "refers to one's ability to reason in terms of general 
structures, not only in terms of their instances" (Harel \& Soto, 2017, p. 231). The ability to reason in this way absolves the student of the need to consider individual explicit examples when solving problems: the ability to reason structurally allows one to think generally about concepts like "groups" without needing to reference specific examples like $\mathbb{Z}_{7}$. Necessarily, the student's concept image (Tall \& Vinner, 1981) directly influences the student's representation(s) of the structure in question. Harel and Soto (2017) reported on "categories of structural reasoning" (p. 227) which embody the properties of structural reasoning, and they described instances of each category as they observed them in empirical studies. However, the category "reasoning in terms of general structures" was not manifested by students in their empirical data. We propose that the ability to reason in terms of general structures is at least beneficial, if not necessary, for the construction of many proofs in advanced mathematics. In this paper, we describe evidence of a student reasoning in terms of general structures by means of structural examples and the ways she leverages that reasoning to support her proof construction.

\section{Methods}

\section{Data Collection}

Our goal for data collection was to observe the ways undergraduates generate and interact with examples and visual representations of concepts when writing proofs in topology. We recruited student volunteers from a semester-long undergraduate-level course in point-set topology at a large university in the United States. Student volunteers participated in weekly, hour-long "group study sessions." A total of nine sessions were held throughout the semester; sessions were not held on weeks during which an exam in the topology class was scheduled. Neither of the authors was the instructor of the topology course. The first author acted as facilitator during each session, probing students to explain their reasoning or what they found 
confusing as they worked through proving tasks. The role of the facilitator was similar to that of a clinical interviewer, though we refrain from using this descriptor as we did not conduct structured clinical interviews. Each group study session was video recorded and transcribed for analysis.

Each group study session was limited to approximately one hour in length. During each group study session, the student volunteers were asked to complete between one and three proofrelated tasks, depending upon the time available and the students' progress on given tasks. The first author attended all class sessions (excluding exams) to determine what content had been covered recently, and proof tasks were chosen to align with content covered recently in class. Proof tasks consisted of three types of tasks: "prove" tasks, which were presented as true statements the students were asked to prove, "disprove" tasks, which were presented as false statements the students were asked to disprove, and "prove or disprove" tasks whose truth value was not given to the students. The order in which the tasks were presented varied among sessions.

These sessions were intended to mimic an authentic homework and studying environment as closely as possible. Furthermore, we hoped that this process would be mutually beneficial for the researchers and for the students. Thus, students were encouraged to collaborate to solve problems and to reference their notes or textbooks when they felt inclined to do so. Students were also permitted and encouraged to discuss their reasoning with the facilitator. The facilitator sometimes provided help to the students in the form of demonstrating or modifying the use of notation and pointing out errors in logic and reasoning.

In total, four students (three undergraduates and one graduate student) participated in our study. The students' mathematical backgrounds and experiences with proving varied; during 
their first session, each student filled out a demographic survey indicating their academic major(s) and minor(s) as well as all mathematics courses they had taken at the college level. In order to maximize our data corpus, students were not required to attend every session, but they were encouraged to attend as many as they were able.

Only one student, to whom we assigned the pseudonym Stacey, attended all sessions. The data presented in this paper focus on Stacey and should be considered as a case study of Stacey's behaviors in example generation and use when working on proof tasks in general topology. Stacey was a second-year undergraduate mathematics major. On her demographic survey, Stacey reported having completed courses in differential, integral, and multivariable calculus as well as a computationally-based introductory course in linear algebra and an introduction to proof course. With this in mind, we assume Stacey's experience with proof writing in general to be limited. However, throughout the course of the study, Stacey mentioned and demonstrated knowledge of or experience with basic proving strategies such as direct proof, proof by contradiction, and disproving by means of a counterexample.

\section{Data Analysis}

Data were initially coded using deductive thematic analysis (Braun \& Clarke, 2006). Our initial coding scheme consisted of three main themes based on types of examples already described in the literature: specific examples (isolated examples not intended to represent any generality), generic examples (Mason \& Pimm, 1984), and algorithmically-generated examples (e.g., Sinclair et al., 2011). Those examples coded as specific were produced as isolated examples with no evidence to suggest that they were intended to represent any generality. Examples coded under this theme were specific in the sense described by Mills (2014): they represented a particular member of a larger class, e.g., the interval $(0,1)$ as a specific example of 
an open set. Examples coded as generic were specific examples Stacey produced along with evidence to suggest that she saw them as representative of a larger class of examples. Stacey demonstrated an awareness of the arbitrariness of these example choices, as in Session 3 when she was asked to produce an example of a constant function, and she shrugged as she said “ $f(x)=1$." Stacey's body language and her tone of voice suggested that she thought of constant functions as " $f(x)=$ some number" and understood that all functions from this class would behave the same way. Algorithmically-generated examples were those that Stacey appeared to have a rule for producing. In Session 4, Stacey conjectured that "an open set taking away finite sets is still open," immediately producing the example $(0,1)-\{2\}$ to demonstrate her reasoning, though it was apparent that taking the set difference between any open interval and any singleton contained within that interval would have worked equally well.

After this initial round of coding, we observed that there were still representations that met our definition of example but did not belong to any of the three themes described in the preceding paragraph. That is, they were manifestations of mathematical objects which satisfied given mathematical definitions, and they appeared to aid Stacey in generalizing or abstracting ideas. These examples were akin to Mills' (2014) generic diagrams, in that they possessed the necessary concreteness for Stacey to investigate the properties of the given definition, but they lacked specificity in that they did not represent specific members of a given class of mathematical objects.

The three existing codes (specific, generic, and algorithmically-generated examples) were grouped under the heading explicit examples. To categorize the other examples, we defined another code called structural examples. A structural example is an example that possesses only 
the essential properties of a definition but lacks any additional properties that might be present in an explicit example.

\section{Results}

We present four instances of Stacey's structural example generation to clarify the nature of structural examples: two during the Prove task and two during the Disprove task. When Stacey produced a structural example in response to a Prove prompt, she was able to use her example to investigate the properties of the object represented in the example. Structural examples generated in response to Disprove prompts gave Stacey insight into which portion of the prompt was false, guiding Stacey's search for a counterexample.

\section{Structural Examples During the Prove Task}

The structural examples Stacey produced in response to Prove prompts in Sessions 5 and 7 represented mathematical objects defined in the prompts. Although the structural examples were not specific in the sense described by Mills (2014), they possessed the requisite concreteness to allow Stacey to explore their properties and how those properties would be affected if the conclusion of each prompt were not true. These explorations informed Stacey's decisions in her formal proof writing, in each case leading her to produce an indirect proof.

In Session 5, Stacey produced a structural example in response to the prompt to prove the following:

Prove: A subset A of a topological space $(X, T)$ is said to be dense in $X$ if $\bar{A}=X$.

Prove that if for each open set $O \in T$ we have $A \cap O \neq \emptyset$, then $A$ is dense in $X$.

The notation $\bar{A}$ denotes the topological closure of $A$. We wish to emphasize Stacey's inexperience with the concept of dense subset; as the word dense was written on the chalkboard, Stacey vocalized, “Ah, I saw something about this, but I haven't done anything about it yet. He’s 
mentioned it I think, but hasn't said..." Furthermore, in her demographic survey, Stacey indicated that she had not taken a course in real analysis; only courses in the introductory calculus sequence, an introduction to linear algebra, and an introduction to proof course. Thus, we infer that Stacey's example space for this concept was sparse at best.

Upon reading the prompt, Stacey began by constructing a diagram (Figure 7a) which was coded as a structural example.
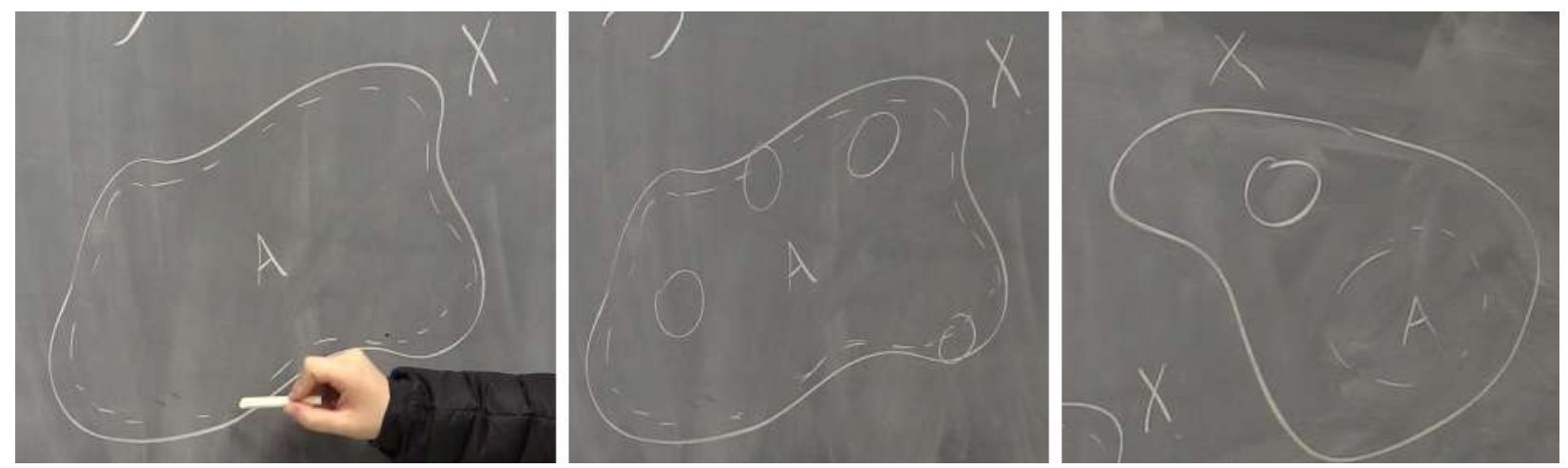

Figure 7(a-c): Stacey's structural examples during the Prove task in Session 5.

She explained:

I can't really show it with a picture because I can't draw, like, a dashed line over a... solid line, but we have $\mathrm{X}$ on the outside, and then we have the set $\mathrm{A}$, which is represented by the dashed, which I wish I could get closer to this [pointing to the border of $X]$, but I can't. So if we had the closure of A, then it would just be the same as that solid line. So then if you take any open set anywhere [drawing circles, Figure 7b], there has to be some kind of intersection with A. So if it wasn't, like if you take, if the intersection could be, the empty set [trailing off, drawing Figure 7c] You've got $\mathrm{X}$ here, and A here, and you could have an open set here, and their intersection would be the empty set. But then this closure wouldn't be equal to X. I get it conceptually I think, but I'm not sure how to prove it. 
This quote demonstrates Stacey's view of this diagram as a representation of a mathematical object (Alcock \& Weber, 2010), namely, a set with a dense subset. Stacey also very clearly used her diagram to investigate the properties of such an object (Mills, 2014), as in her construction of Figure $7 \mathrm{c}$ when she considered the ramifications of the existence of an open set whose intersection with $A$ was empty. Thus, we identified this diagram as an example. Stacey continued, and based on her reasoning using Figure 7c, proved the statement by way of contradiction.

The most apparent feature of this example is its abstractness; this example possesses all the necessary criteria (as Stacey conceptualizes them) to be considered a representation of a set with a dense subset, but without any additional properties that might allow us to identify it as a specific case. As such, this example may be viewed as a skeleton (Leron, 1985) of a specific example, since any specific example must possess the properties represented in this structural example. But the example lacks any dimensions of possible variation, such as cardinalities or identities of the sets.

Stacey exhibited similar behavior in Session 7 when asked to prove the following:

Prove: Let $(X, T)$ be a topological space. A separation of $X$ is a pair $U, V$ of disjoint open subsets of $X$ whose union is $X . X$ is connected if no separation of $X$ exists. If the sets $C, D$ form a separation of $X$, and if $Y$ is a connected subspace of $X$, then either $Y \subseteq C$ or $Y \subseteq D$.

As in Session 5, Stacey began by drawing a diagram (Figure 8a), which was coded as a structural example. 

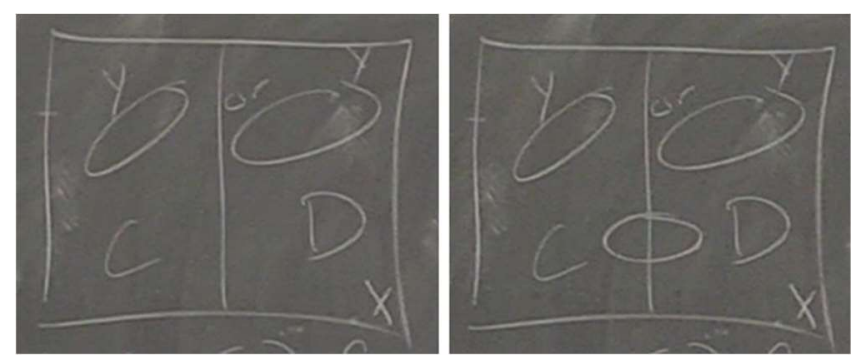

Figure 8(a-b): Stacey's structural examples during the Prove task in Session 7.

After completing the diagram, Stacey began to work on a proof, but she stopped herself to explain: If you have $X$, which is also the ambient space here, and then you have the sets $C$ and $D$, they form a separation. That means that they're disjoint, so they don't have any of the same elements, and that their union is $X$, so that is satisfied for this. And then if $Y$ is connected, which means it's not in these sets that are disjoint whose union is $Y$, it's just one cohesive set, then it has to be either in $C$ or in $D$. It can't be in both [pause] because if it was like that [draws Figure 8b], it would be disjoint [misspeaking: disconnected].

As in Session 5, Stacey then proved this statement by way of contradiction. While the concept of connectedness had been covered recently in Stacey's topology class, it was introduced there with the following definition: "A topological space $X$ is connected if the only sets that are both open and closed in $X$ are the empty set and $X$, itself." Although Session 7 was likely Stacey's first exposure to this particular definition and to the definition of separation, it is less likely that her concept image and example space related to connectedness were as much of a blank slate as those related to the idea of a dense subset in Session 5.

Nevertheless, the structural example Stacey generated was consistent with the definition of separation and represented only the essential property that the topological space is composed of the union of two disjoint open sets, along with the conclusion of the stated conjecture. Again, 
Stacey was able to use this example to investigate the properties of such an object and to explore the consequences of an open set intersecting both parts of a separation. The example is concrete in the sense that it can be used for such experimentation, though it lacks specificity as it does not identify a particular separation of a particular set.

\section{Structural Examples During the Disprove Task}

Stacey generated structural examples during Sessions 5 and 7 in response to the Disprove prompts as well. In each case, Stacey then used her structural example to identify which portion of the statement was problematic and would lead to the construction of a counterexample.

In Session 5, Stacey was asked to disprove the following statement:

Disprove: Let $(X, \mathcal{T})$ be a topological space, and let $A \subseteq X$. Define the boundary of $A, \operatorname{Bdry}(A)$, by $B d r y(A)=\bar{A} \cap \overline{C(A)}$. Then $B d r y(A)$ is both open and closed in $X$.

Stacey immediately produced the structural example in Figure 9, explaining...

So we have, $A$ is gonna be a subset of the closure. So if we have, this is $A$ [drawing a circle with a dashed boundary], then if you make the closure, you just close up that boundary [drawing a solid boundary over the dashed one, Figure 9a]. But this would now be the closure of $A$; you take all the arbitrarily close points to $A$ and include them in $A$ [miming scooping points toward the center of A, Figure $9 b$ ]. So then, this is $A$ again [drawing another circle with a dashed boundary], just the complement of $A$ would be all the stuff around it [drawing a larger circle with a solid border around the dashed circle and shading in between], and then you would include that boundary of $A$ for the closure of the complement of $A$ [drawing a solid circle over the dashed one, Figure 9c]. So then the intersection of the closure of the 
complement and the closure of $A$ would just be this line, this... on the outside here [shading the border of the first, larger circle, Figure $9 c$ ]. I think it would just be closed, not open and closed. Not sure how to prove that, but I think it would just be closed.
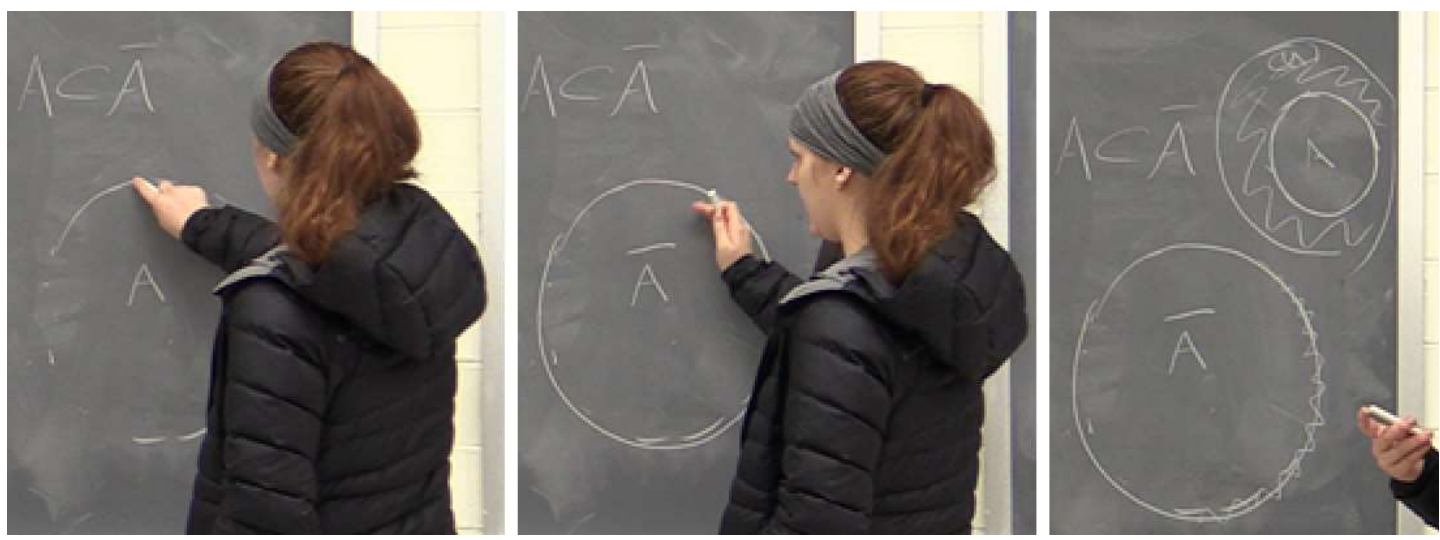

Figure 9(a-c): Stacey's structural examples during the Disprove task in Session 5.

Stacey then recognized immediately that the boundary of A must be closed, although she appeared to lack confidence in this idea at first. She quickly progressed in reasoning from what appeared to be an intuitive snap judgment, through recognition that the intersection of two closed sets is closed, and finally to writing a formal proof that the boundary of A must be closed. Consequently, it became clear to Stacey that she needed to prove that the boundary of A need not be open.

Though it became clear later (via direct questioning by the facilitator) that Stacey was aware of the utility of a counterexample for disproving false statements, Stacey did not immediately attempt to find a counterexample. Rather, the discussion turned toward possible methods to prove directly that the boundary of A must not be open. Due to our interest in students' organic behaviors, the facilitator allowed the conversation to continue in this direction for a few minutes as Stacey discussed possible routes for writing such a proof, such as showing that the 
complement of $B \operatorname{dry}(A)$ was open (Stacey frequently struggled with the idea that a set might be both open and closed) or that $\operatorname{Bdry}(A)$ satisfied the definition of an open set.

After a few minutes, the facilitator posed the question, "How do you prove that something is false?" prompting Stacey's immediate reply of “Counterexample." Stacey thought for a moment, then asked "Could we just do something in $\mathbb{R}$ ?" She drew a number line and labeled the points 0 and 1 , then indicated that she wished to consider $A=(0,1)$ and explained that $\operatorname{Bdry}(A)=\{0,1\}$ was not an open set because she could not draw a ball around either point that would be contained entirely within $\operatorname{Bdry}(A)$ (Figure 10).

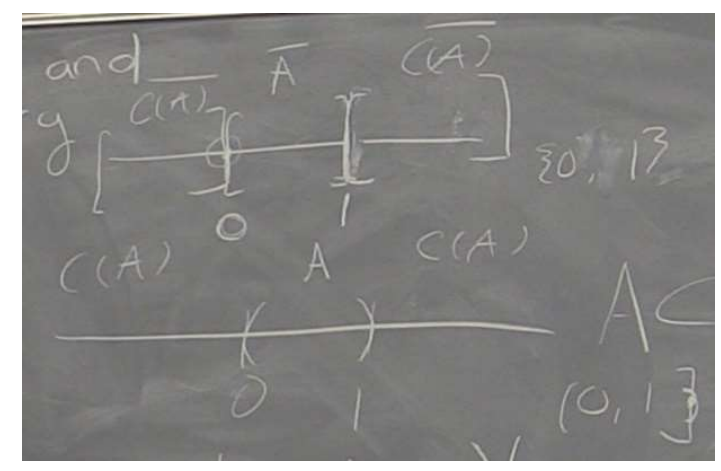

Figure 10: Stacey's counterexample in Session 5.

The diagram Stacey drew in this scenario represented the intersection of a set, $A$, and its complement, and it seemed to facilitate Stacey's reasoning about the properties of that intersection. Stacey's structural example did not appear to play a direct role in her eventual identification of an appropriate counterexample. However, it does appear that her construction of the structural example led her to determine the properties such a counterexample should have, namely, that her counterexample should be a set for which the intersection of its closure and the closure of its complement should not be an open set.

Stacey's Disprove prompt in Session 7 was the following: 
Disprove: Let $(X, \mathcal{T})$ be a topological space. Let $A \subseteq X$. If $D$ is a connected

subspace of $X$ that intersects both $A$ and $C_{X}(A)$, then $D \cap B d r y(A)=\emptyset$.

While reading through the statement, Stacey extracted some of the information into a list of notation "so [she] could understand it better," then she drew the diagram in Figure 11.

Immediately upon finishing her sketch, she looked at the problem statement, pointed at the text " $D \cap B d r y(A)=\emptyset$," and said "So that has to be the false part" while looking back and forth between the text and her diagram. She underlined this portion of the problem statement and wrote the word false beneath it. Referencing her diagram, she explained that if $D$ must intersect both $A$ and the complement of $A$, then "there's no way that it also doesn't intersect with the boundary."

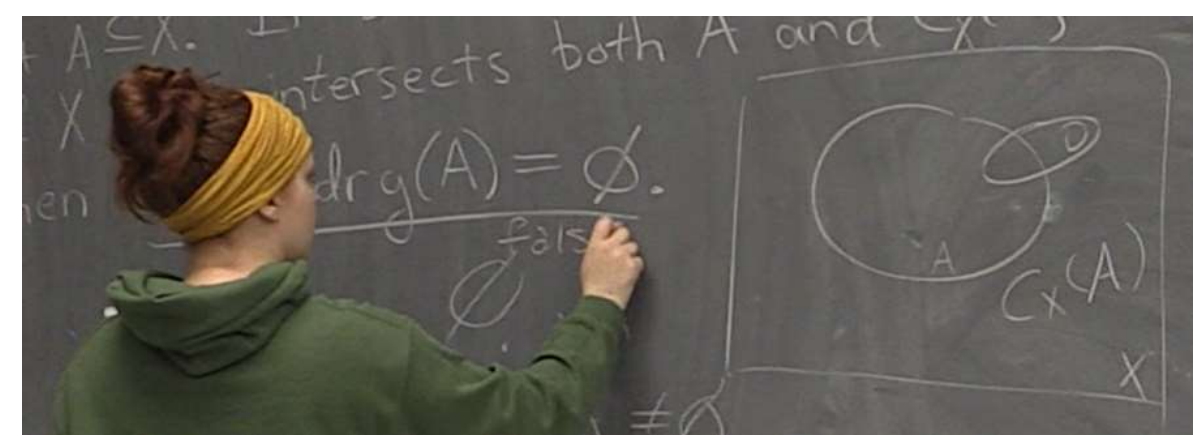

Figure 11: Stacey labeling the false portion of the Disprove prompt in Session 7, beside her structural example.

Stacey continued, "So we could give an example in $\mathbb{R}$ squared." She then sketched a pair of intersecting circles on a set of coordinate axes and reasoned about the pair of disks $x^{2}+y^{2} \leq$ 2 and $(x-1)^{2}+(y+1)^{2} \leq 2$ as specific instances of the sets $A$ and $D$, respectively (Figure 12). As she reasoned about this pair of disks, Stacey modified her original diagram to make the set $D$ more circular, similar to the smaller diagram she drew above it to represent the disks she chose for her counterexample. She explained,

If you have, this is A and A is the disk, this disk with center $(0,0)$ with radius square root of 2, and you also take, this is D, and it satisfies D intersect with A 
isn't empty, and D intersect with the complement of A isn't empty, but it does intersect the boundary.

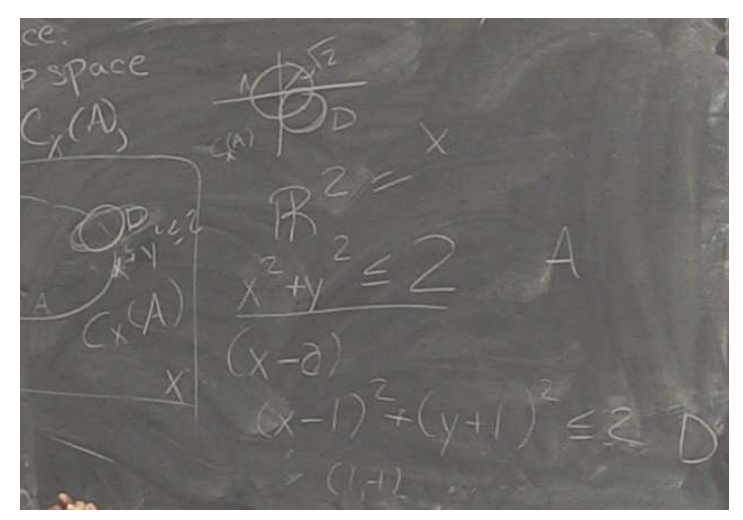

Figure 12: The disks with radii $\sqrt{2}$ centered at $(0,0)$ and $(1,-1)$.

As in Session 5, upon completion of her structural example, Stacey immediately recognized which portion of the given statement must be false, namely that $D \cap B d r y(A)$ must not be empty. It is unclear in this case the extent to which her example influenced her choice of a specific counterexample. It is worth noting, however, that the sketch of the counterexample chosen in this case bears a striking visual similarity to the structural example Stacey produced, and Stacey modified her original drawing to look more like the sketch she produced of the disks she chose for her counterexample. Much more computationally simple counterexamples to this claim exist in $\mathbb{R}$, so it is not unreasonable to suspect that Stacey's choice of counterexample may have been influenced in this case by the salient visual elements of her structural example. What seems clear, however, is that Stacey's construction of a structural example helped her identify the property in the prompt that her counterexample must show need not hold.

\section{Discussion}

Our definition of example, adapted from Watson and Mason (2005) and Alcock and Weber (2010), is "any mathematical object satisfying the definition of some concept, from which it is expected to generalize or to investigate the properties of the object" Our data show that 
Stacey's structural examples fit this definition, although they lack the specificity that Mills (2014) required of her examples. However, Stacey's structural examples did demonstrate concreteness, which she leveraged in proving true statements to explore the properties of her examples. Her structural examples also helped her identify counterexamples by helping her determine which property from the conclusion of a false statement must be violated by her chosen counterexample. We conclude our discussion with a comparison of explicit examples and structural examples in terms of their dimensions of possible variation and ranges of permissible change.

\section{Using Structural Examples to Help Prove True Statements}

Recall that a structural example is an example that possesses all the essential properties of a given concept but no other properties which may be possessed by explicit examples. Structural examples are therefore extremely abstract; they act as a sort of template for explicit examples, representing every property that must be common to all explicit examples. Stacey produced these objects and used them to explore the relevant abstract mathematical structures in Sessions 5 and 7 (i.e., a set with a dense subset and a connected subspace of a disconnected topological space). Stacey was able to convince herself of the truth of each given statement by exploring the properties of her example and using her example to investigate what would break down if the conclusion failed to hold (Gallagher \& Engelke Infante, 2019; Harel \& Sowder, 1998; Mills, 2014; Raman, 2003). Using her structural examples in this way, she quickly determined why the given statement was true as well as a starting point for how to prove it.

This is exactly the kind of structural thinking required for proof construction; one must be able to strip a concept down to its essential components and derive logical conclusions from them. Harel and Soto (2017) described several kinds of structural thinking; among them were 
"recognizing and operating with structure in thought... epistemological justification... [and] reasoning in term[s] of general structures" (p. 232). Stacey's structural examples provide evidence of her abilities to recognize structure and reason in terms of general structures. This recognition of abstract structure led Stacey to produce proofs in a manner similar to Leron's $(1983,1985)$ structural method of proof writing. Leron presents the structural method as a method of proof delivery, not construction, in which big ideas and overarching themes of the proof are presented in the first layer, and subsequent layers of the proof expand upon these ideas, filling in details. Stacey's structural examples helped her develop proofs in a structural style akin to Leron's method, helping her recognize the key ideas of her proofs (Gallagher \& Engelke Infante, 2019; Raman, 2003) which served as that first layer, which she was then able to expand upon in her formal proof writing. The key idea of each proof then serves as Stacey's epistemological justification for the creation of the structural example, as the recognition of the key idea justified the creation of the example.

\section{Using Structural Examples to Identify Counterexamples}

Though Stacey's structural examples lacked the specificity to constitute counterexamples, they appeared to help her identify which property in a given statement must fail to hold. This is often the first step in educated counterexample generation: knowledge of what properties one wants and doesn't want to be present in a counterexample facilitates the selection of one option out of many potential options. Thus, Stacey's structural examples acted as scaffolds to help her identify appropriate counterexamples.

Throughout the course of this study, Stacey’s concept image (Tall \& Vinner, 1981) of an open set as a region in which each point can be contained within a ball became apparent. This concept image manifested in Session 5, particularly as she discussed the counterexample she 
chose, $A=(0,1)$, and explained that the boundary of this set, $\operatorname{Bdry}(A)=\{0,1\}$, wasn't open because she couldn't put a ball around either 0 or 1 that would be contained within the boundary of $A$. Her concept image of closed sets and closure seemed to include drawing solid boundaries around closed sets to symbolize including all the points "arbitrarily close," while open sets should be contained within "dashed" curves. In Session 5, It appeared that this imagery, along with her structural example, helped Stacey to intuitively identify that the boundary of a set should be closed but not necessarily open. Her confidence in this claim grew as she discussed it, as she progressed from "I think it would just be closed, not open and closed. Not sure how to prove it, but I think it would just be closed," through "if we're intersecting two closed sets we're gonna get a closed set," and finally to a full proof of the fact that $B \operatorname{dry}(A)$ is always closed. Thus, it became clear to Stacey that the counterexample she needed to find was one whose boundary was not an open set.

Stacey's concept image of connected and disconnected sets seemed to include the absence or presence of a clear line of separation dividing a set into two sets sharing a boundary. When she drew her structural example to represent a set $A$ and a connected set $D$ intersecting both $A$ and its complement, it was immediately apparent to Stacey that $D$ must intersect the boundary of $A$. The impact of her structural example on her decision-making was clear, as she pointed to " $D \cap \operatorname{Bdry}(A)=\emptyset$ " in the problem statement and announced that it "had to be the false part" while looking between her diagram and this part of the claim. Also of note is the visual similarity between the geometric features of Stacey's structural example and the counterexample she would later produce, both taking the shape of two intersecting disks.

While we do believe that Stacey's structural examples helped her with counterexample identification by clarifying which property in the conclusion of a statement must fail, it is not 
necessarily the case that they made it clear what specific counterexample would demonstrate this failure, nor even that it was always clear that Stacey should look for a counterexample. Note that in Session 5, even after Stacey determined that the boundary of $A$ need not be open, she initially sought a direct proof (which would prove that the boundary of $A$ cannot be open) rather than a counterexample (to show that it may not be open). It is not clear from our data whether Stacey was conscious of the difference in the implications of these two approaches, but it was not until the facilitator asked her directly "How do you prove that something is false?" that she began to talk about finding a counterexample.

We note as well that the textbook used in this course (Mendelson, 2012) did not contain images of the type used by Stacey in the instances described above. That is, the textbook did not contain images of open sets containing points inside balls, representations of open sets as regions with dashed borders, or disconnected spaces as regions with a dividing line. The textbook used in Stacey's calculus courses (Stewart, 2016) also did not use any of these images in its discussion of open or connected regions. Though the first author attended lecture sessions for Stacey's topology class, data were not collected with respect to the types of imagery used by the instructor, as the main purpose of attendance was to determine appropriate content for the group study sessions. Thus, we cannot point to the origins of Stacey's mental images with any certainty.

\section{Dimensions of Possible Variation and Range of Permissible Change}

Example generation in mathematics serve two purposes. One of these purposes is clarification: mathematical language often differs from everyday speech, and examples can be used to decode the meanings embedded in definitions and theorems (Vinner, 2002). The other purpose served by example generation is the identification of elements of structure. Examples 
provide demonstrations of what can change and what can't - the dimensions of possible variation and that which must be invariant in the midst of change (Mason \& Watson, 2008). The distinctions among the various types of explicit examples (specific, generic, and algorithmicallygenerated examples) and structural examples may be elaborated through a careful consideration of the attention given to the dimensions of possible variation and their corresponding ranges of permissible change during example generation.

When asked to provide examples of irrational numbers, many students may simply respond with a memorized list of numbers, $\sqrt{2}, \sqrt{3}, \pi, e$ being common choices. These are specific examples, as they aren't intended to represent any generality, but they do represent particular members of the class of irrational numbers. Such a student likely is not keenly aware of irrational numbers as those that cannot be represented as a ratio of integers, nor will they be able to produce additional examples without great effort. A list like the one above does not give any insight into the structure underlying irrational numbers: it shows no true understanding of the properties of irrational numbers nor how they might be found or their relationships with rational numbers or other irrationals. In providing a list of irrational numbers like the one above, such a student does not attend to the dimensions of possible variation among irrational numbers, nor to the corresponding ranges of permissible change, likely because the student is not yet aware of them.

Of course, it is not always necessary to have access to an extensive list of examples in order to have some sense of structure. Some students may also produce only a few examples of irrational numbers, such as those described in the preceding paragraph. However, they may be able to reason about properties of these examples, noting that although they can only produce a few examples, they "get the idea." These students can recognize that any other examples they 
might find would be similar to these in a few key ways, perhaps in their inability to be expressed as ratios of whole numbers or as terminating or repeating decimals. They may even have a sense that "taking a square root" is somehow tied to this idea, but that this isn't all there is to it since taking the square root of numbers like 4 and 9 fail to produce irrational numbers. For these students, examples like $\sqrt{2}$ are generic; they can see through the example to understand the properties it exemplifies. Such a student is attending to the dimensions of possible variation, but not the ranges of permissible change. They have some awareness that changing the number under the radical may produce additional examples, but not just any number will do. More importantly, they understand that they likely won't gain much (if any) additional information by generating additional examples, as they have achieved some insight into the underlying structure of irrational numbers.

Still other clever students might notice that taking the square root of a prime number will always produce an irrational number. Examples like $\sqrt{2}$ and $\sqrt{3}$ become algorithmicallygenerated; they are specific, but now can be seen to belong to a larger class of examples, an unlimited number of which can be produced as needed. These students are aware of both a dimension of permissible change and its range of permissible change, as they recognize not only what can change to produce additional examples but also the extent to which it can change. Of course, this example generation process cannot generate every irrational number, but a student with this knowledge possesses an algorithm for generating as many irrational numbers as desired.

Because structural examples possess only the essential properties of a concept and nothing more, they show a lack of attention to dimensions of possible variation and their ranges of permissible change, as changing any property of the example destroys its "examplehood." 
What might have prompted Stacey to produce structural examples instead of explicit examples? One possibility is that Stacey is unaware of the dimensions of possible variation. In Session 5, for example, Stacey encountered the definition of a dense subset for (we believe) the first time. It is not unreasonable to think that finding an explicit example might be challenging for her since she has never had the intellectual need to explore the dimensions of possible variation (Harel \& Soto, 2017; Marton \& Tsui, 2004). Another possibility is that Stacey realizes that, for the purpose of writing a proof, the dimensions of possible variation and their ranges of permissible change often don't matter. With the exception of proof by exhaustion, proof writing typically does not require the consideration of a wide variety of cases. Rather, proof writing requires attention to the essential structure of a mathematical construct.

What distinguishes structural examples from specific examples is the attention to structure. Specific examples are often memorized and simply regurgitated without any real understanding. Structural examples, on the other hand, are generated, and demonstrate an awareness of the underlying structure of the example, as the inessential details of the example have been stripped away. The structural example highlights what is invariant in the midst of change (Mason \& Watson, 2008). It demonstrates understanding of the "big ideas" behind concepts even if the details are unclear or unnecessary.

Awareness of what is invariant in the midst of change is exactly what is needed for proof construction. When writing a proof, these are the only properties that can be used to derive a logical conclusion. Knowing what is invariant in the midst of change is essential for identification of appropriate counterexamples as well. When one is presented with a conjecture, a certain set of properties is specified in the hypothesis and in the conclusion. Being aware that the properties given in the hypothesis must not change and what those properties logically imply 
reduces the list of properties the prover must try to invalidate and highlights those which may be problematic.

\section{Conclusions}

With this research, we addressed the following research questions: what kinds of examples do students use when writing proofs in topology, and how do they use them? We described an abstract kind of example that we called structural examples used by one of the students in our study. Based on our case study of Stacey, structural examples appear to be a productive tool for proof construction and counterexample generation in general topology. They can demonstrate a student's understanding of the underlying structure of abstract mathematical objects, and their lack of properties inessential to the given definition prevents the student from being distracted by these properties, providing a great benefit in proof writing. Furthermore, structural examples appear to help students quickly identify the troublesome properties in false statements, helping them determine appropriate counterexamples.

Additional studies are needed to determine whether structural examples are widely useful for students writing proofs in general topology or if Stacey's is an isolated case. If structural examples do prove to be generally useful, then further studies must determine effective strategies for teaching students to generate such examples and to use them to extract useful information for their proofs, as simply having a visual representation of a concept does not guarantee its utility in problem solving (Booth \& Thomas, 2000; Mason, 1992). Another interesting question is whether this behavior is common in other areas of mathematics such as abstract algebra, graph theory, and calculus, or if there is something unique about topology that encourages this behavior or makes it useful. We believe it is not unreasonable to suspect that the geometric nature of this field has an influence on students' approaches to solving problems and is hence worthy of further 
investigation. Furthermore, Stacey did not always produce structural examples: are certain topics within topology more amenable to this strategy than others, and if so, why might that be the case? In any case, structural examples provide a powerful tool for informal assessment of students' understandings of the underlying structure of abstract mathematical objects. Structural examples serve to expand our understanding of students' example use in proof writing and may have great practical implications for the teaching and learning of topology. 


\title{
PAPER 3: THE COMBINED EFFECT OF GESTURE USE AND DIAGRAM CONSTRUCTION ON PROOF WRITING IN TOPOLOGY
}

\begin{abstract}
Proof writing is notoriously difficult for students to master. In this paper, we present a case study of Stacey, an undergraduate writing proofs in introductory topology. We provide an analysis of Stacey's proof constructions that involved the use of diagrams. We view our data through the combined lenses of embodied cognition and commognition, and we show that Stacey's success was largely due to a combination of verbal communication, diagram construction and use, and gesture use. We argue that Stacey's grounding of abstract mathematical ideas in diagrams facilitated her applications of cognitive schema for physical relations to the mathematics. Furthermore, we propose that Stacey's communication of her ideas through words and gestures transformed her ideas. This combination of communication and application of cognitive schema resulted in Stacey's recognition of the key idea of proof, ultimately leading to successful proof construction.
\end{abstract}

\section{Introduction}

Mathematical proving and problem-solving relies heavily on the use of symbols, such as mathematical notation, diagrams, and gestures. Symbols can be used as "repositories of complexity" (Sfard, 2009, p. 197), storing complicated ideas in a compact form. However, more than just storing information, symbols can be used to share and transform information through communication and cognition (Comi \& Eppler, 2011). In this paper, we present a case study of Stacey, an undergraduate in an introductory course in general topology. We observed that, when writing topology proofs, Stacey utilized a combination of speech, gestures, and diagrams to identify the key idea of the proof (Raman, 2003), at which point she was able to translate that 
idea into a formal proof. We begin with a discussion of the literature on proofs, diagram use, and gesture use in mathematics.

Writing mathematical proofs is known to be challenging for students (Alcock \& Weber, 2010; Azrou \& Khelladi, 2019; Harel \& Sowder, 1998; Iannone \& Inglis, 2010; Leron, 1983, 1985; Moore, 1994). Various potential reasons for students' difficulties with proof writing have been proposed and explored, including a lack of content knowledge (Azrou \& Khelladi, 2019; Moore, 1994), lack of strategic knowledge and knowledge of proof techniques (Weber, 2001), difficulty interpreting the meaning of complex logical statements (Zandieh et al., 2011), and even a fundamental difference between the values of mathematicians and students (Dawkins \& Weber, 2016).

Students often spontaneously produce graphical arguments as part of their proving processes (Booth \& Thomas, 2000; Gallagher \& Engelke Infante, 2019; Harel \& Sowder, 1998; D. Zazkis et al., 2016; Zhen et al., 2016). Zhen et al. (2016) demonstrated that students are often aware that graphical arguments, on their own, do not constitute mathematical proof, but they believe that such arguments do contain information that can be useful for proof writing. Zazkis et al. (2016) examined the work of students who were successful in translating graphical information into a formal, deductive argument and identified behaviors that seemed to support the success of these students. In our previous work, we determined that Stacey's visual representations played a role in her identification of the key idea of a proof (Gallagher \& Engelke Infante, 2019). In the current study, we build on this work, showing that it was not just Stacey's diagrams, but rather a complex interplay among her diagrams, gestures, speech, and cognition that led her to recognize the key ideas of several proofs. 
Gesture use and perception are known to have direct connections to language and cognition (Alibali et al., 2014; Bernard et al., 2015; Goldinger et al., 2016; Hostetter \& Alibali, 2008; Lakoff, 2012; Lakoff \& Núñez, 2000; McNeill, 1992, 2005; Straube et al., 2011; Varela et al., 1993; Wilson, 2002). Studies using fMRI have demonstrated that there is a direct connection between the interpretation of language and spatial information and the production and perception of gesture in the brain (Bernard et al., 2015; Straube et al., 2011). The embodied cognition perspective argues that cognition, physical perception, and action are all intimately connected, as knowledge is built from perception of external stimuli, and gesture and communication exist for the purpose of giving embodiment to mental images and ideas (Goldinger et al., 2016; Lakoff, 2012; Lakoff \& Núñez, 2000; Varela et al., 1993; Wilson, 2002). Sfard's $(2001,2009)$ theory of commognition proposes that, rather than being separate processes, thought, gesture, verbal communication, and symbol use are really parts of a larger whole. In this paper, we will answer the following research question: "How do students synergize cognition, gestures, diagrams, and communication when writing proofs in topology?"

\section{Theoretical Perspective}

To discuss the role that gestures and diagrams played in Stacey's construction of topology proofs, we approach our data from a hybrid of the embodied cognition perspective (Lakoff \& Núñez, 2000; McNeill, 1992, 2005; Varela et al., 1993) and the theory of commognition (Sfard, 2001, 2009).

According to the theory of embodied cognition, the mind does not work on purely abstract entities with no counterpart in reality; rather, mental actions are inextricably linked to physical actions and perceptions (Barsalou, 1999, 2008; Glenberg \& Robertson, 2000; Goldinger et al., 2016; Lakoff \& Núñez, 2000; Varela et al., 1993; Wilson, 2002). Therefore, mathematical 
ideas are not purely abstract, but they derive from physically substantiated ideas, and in turn must be able to be expressed physically. "To understand a mathematical symbol is to associate it with a concept - something meaningful in human cognition that is ultimately grounded in experience and created via neural mechanisms" (Lakoff \& Núñez, 2000, p. 49).

In the embodied perspective, mathematical ideas are often conceptualized via schemas, such as the Container schema and the Source-Path-Goal schema. Schemas are cognitive structures with a particular set of properties (Lakoff, 2012; Lakoff \& Núñez, 2000). The Container schema, for example, has a structure consisting of an interior, a boundary, and an exterior, with the understanding that an object is "in" the container if the object is located in the interior. We can apply the Container schema when we recognize that an object or situation we have encountered has a similar structure, and we can apply the same logic and language to that situation. Schemas give us mental shortcuts to processing complicated information by fitting that information into a pattern we already understand. A common example of the application of the Container schema to mathematics is the idea of sets. A set acts as a container with a defined interior and exterior, the boundary between which being defined by the defining property of the set. Thus, we apply the same kind of language as we might with a container, talking about "elements inside the set" and "subsets contained within larger sets."

The acknowledgement of cognitive schemas makes it much easier to see the influence of embodied cognition in mathematical thought: the schemas representing Containment, Near-Far, Contact-Noncontact, Toward, and many others are used frequently in mathematics, and have obvious connections to the physical world. With the prevalence of these grounded schemas in mathematical reasoning, it should come as no surprise that we should unconsciously assign 
physical properties to the abstract when reasoning about limits, sets, functions, and numerous other concepts and processes in mathematics.

It should also be unsurprising that we should gesture when reasoning about mathematics. The regions of the brain responsible for language processing and gesture processing overlap, already suggesting that gesture should occur spontaneously alongside speech (Alibali et al., 2014; Bernard et al., 2015; Hostetter \& Alibali, 2008; Lakoff, 2012; Lakoff \& Núñez, 2000; Straube et al., 2011). Add to this the fact that mathematical reasoning is inherently based on and schematized in terms of the physical world, and you obtain a complex semiotic system in which physical gestures derive meaning from and provide meaning to abstract mathematical entities.

Due to the complex interrelationship between gestures and verbal communication and the fact that one seldom occurs in the absence of the other, we wish to consider gestures and verbal communication as part of a larger whole. Sfard (2009) refers to this gestalt as commognition. Commognition is a neologism derived from the words communication and cognition. The combination of these two words owes to Sfard's perspective that thinking and communicating are two sides of the same coin: what is generally referred to as communication is thought of in this perspective as interpersonal communication, while thinking is conceived of as intrapersonal communication, or communication with oneself (Sfard, 2001, 2009). Furthermore, in this view, gesture and speech are also seen as two parts of a larger whole, as they both serve the purpose of enhancing communication. Hence, in adopting this view, we presume that speech and gesture are not only tools for interpersonal communication, but they are inherently part of the process of cognition as well.

Because of our interest in Stacey's use of diagrams, inscriptions, spoken words, and gestures to reason about mathematics, we view her mathematics as a semiotic bundle. A semiotic 
bundle is made of signs, such as words (oral and written), gestures, drawings, graphs, and technological devices, that are produced by people engaged in a discourse. Arzarello, Paola, Robutti, and Sabena (2009) noted,

The novelty of the semiotic bundle...is that it allows us to describe the multimodal semiotic activity of subjects in a holistic way as a dynamic production and transformation of various signs and of their relationships. In particular, it properly frames the role of gestures in mathematical activities. (p. 100)

This perspective allows us to consider Stacey's words, gestures, and diagrams as part of a larger whole and to consider their combined impact on her reasoning.

\section{Methods}

\section{Data Collection}

For this study, we recruited a total of four student volunteers from an introductory undergraduate course in general topology at a large university in the United States. These students participated in weekly, one-hour group study sessions during which they were asked to complete proving tasks related to topology. All group study sessions were video recorded and transcribed. The first author acted as facilitator for all group study sessions, presenting problems and occasionally prompting students to explain their reasoning. The first author attended all class sessions (excluding exams) to determine timely and appropriate topics for the proof tasks in each session; proof tasks were contemporaneous with classroom content. Classroom data were not collected with regard to the instructor's delivery of content or proving behaviors.

Proof tasks in each group study session consisted of 1-3 problems, depending upon how quickly the students progressed through each task. Three types of problem were discussed in each session: "Prove" problems, which were given as true, and the students were asked to prove, 
"Disprove" problems, given as false, and the students were asked to disprove, and "Prove or Disprove" problems, whose truth value was not specified. The order in which the problems were presented to the students varied between sessions.

\section{Data Analysis}

Student volunteers were encouraged, but not required, to attend all sessions. Thus, each session was attended by 1-4 students, but the number of students varied. Only one student, Stacey, was present for all sessions. Due to the relatively large amount of data we obtained from Stacey, the results presented in this paper focus specifically on her behaviors. A qualitative case study methodology (Cohen et al., 2013; Yin, 2003b) was applied to determine how Stacey used gestures and diagrams in her attempts to write proofs.

Because of our interest in the interplay between diagrams and gestures, we first identified sessions in which Stacey produced diagrams. For the purposes of this report, we chose to focus our analysis only on cases in which Stacey produced a diagram during her work on the Prove task. Within these instances, we coded our transcripts to identify when Stacey produced gestures while working on a proof. For the discussion of our data, we define a gesture as "movement made by the hand with a specific form: the hand(s) begin at rest, moves away from the position to create a movement, and then return to rest" (Rasmussen, Stephan, \& Allen, 2004, p. 303), a definition adapted from (Roth, 2001). We further classified Stacey's gestures according to McNeill's dimensions of iconicity, metaphoricity, and deixis (Kendon, 2004; McNeill, 1992, 2005). Deictic gestures are pointing gestures. We identified both static points, in which Stacey pointed to something and held her hand or finger in place, and tracing points, in which Stacey pointed to something and then moved her hand along it, as in tracing the border of a set in a diagram. Iconic gestures are those which represent real-world objects and actions, while 
metaphoric gestures represent abstract ideas. For consistency, all representational gestures (i.e., iconic and metaphoric gestures) that referred to abstract mathematical objects such as sets, points, or functions, were coded as metaphoric gestures, since the referents were abstract and not concrete. As in McNeill (2005), we believe these dimensions are not disjoint; thus, a single gesture was sometimes coded with multiple dimensions.

Our previous work showed that Stacey's diagram use was linked with her recognition of the key idea of the proof (Gallagher \& Engelke Infante, 2019; Raman, 2003). Hence, we also identified the moment in each session when Stacey appeared to recognize the key idea of the proof. The key idea is "an heuristic idea which one can map to a formal proof with appropriate sense of rigor" (Raman, 2003, p. 323). We operationalized this definition by identifying the moment when Stacey first verbally expressed an idea that she would later translate into a formal proof.

\section{Results}

We describe three instances in which Stacey produced diagrams as part of her proving process in the Prove task. In two of these sessions, Stacey demonstrated evidence of having a heuristic idea from which to start reasoning about the problem. During these sessions, Stacey expressed her idea verbally, and these verbal descriptions were accompanied by alternation between diagram construction and modification and gesture production. In these two sessions, the combination of communicating verbally, through gesture, and through diagram construction led Stacey to identify the key idea of the proof and subsequently to generate a correct proof. In the third session, Stacey did not recognize the key idea. However, the combination of Stacey's verbal communication and her inscriptions helped her to refine her reasoning in the pursuit of the key idea. 
In Session 5, the Prove task was to show the following:

Prove: A subset A of a topological space $(X, T)$ is said to be dense in $X$ if $\bar{A}=X$.

Prove that iffor each open set $O \in T$ we have $A \cap O \neq \emptyset$, then $A$ is dense in $X$.

The notation $\bar{A}$ was used to indicate the topological closure of the set $A$. After reading the prompt, Stacey immediately drew the diagram in Figure 13 (left).
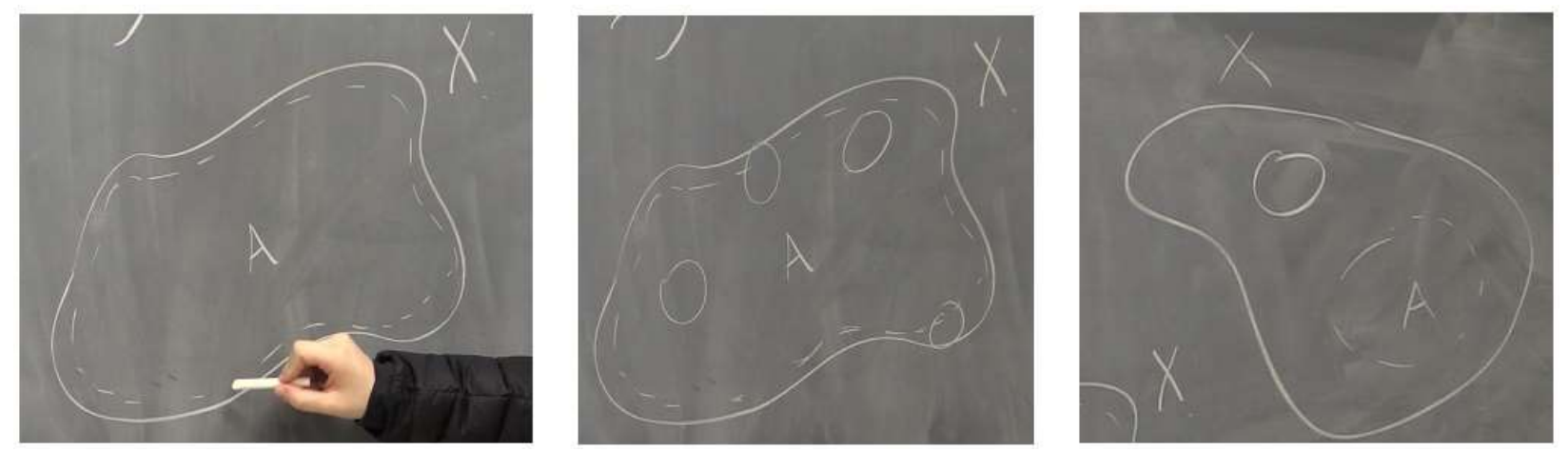

Figure 13: Stacey's diagrams in Session 5.

After she finished drawing her diagram, Stacey reasoned while looking between the written problem statement and her diagram,

I can't really show it with a picture because I can't draw a dashed line over a ... solid line, but we have $X$ [static point to the label $X$ (left)] on the outside [tracing point along the boundary of $X(\mathrm{left})]$ and then we have the set $A$ [static point to the label $A$ (left)] which is represented by the dashed [tracing point along the boundary of $A$ (left)], which I wish I could get closer to this [static point to the border of $X($ left)], but I can't. So, if we had the closure of $A$ [tracing a short line over the label A with her hand, indicating the notation $\bar{A}$ (left)], then it would just be the same as that solid line [tracing point along the border of $X$ (left)]. So then if you take any open set [drawing circles on her diagram (center)] anywhere, there has to be some kind of intersection with $A$ [static point to one of her open sets 
(center)]. So if it wasn't, like if you take, if the intersection could be ... the empty set [static point to $\varnothing$ in the problem statement] - [drawing the diagram in Figure 13 (right)] You've got $X$ here, and $A$ here, and you could have an open set here, and their intersection would be the empty set [recognizes key idea]. But then this closure [static point to the boundary of $A$ (right)] wouldn't be equal to $X$ [static point to the boundary of $X$ (right)]. I get it conceptually I think, but I'm not sure how to prove it.

In this excerpt, Stacey began her reasoning process by drawing a diagram. During her explanation, Stacey did not look at the facilitator nor at Tom, the other student present for this session. Rather, it seemed as though she was talking to herself, thinking aloud rather than trying to communicate anything to the others in the room. As she explained the components of her drawing, she interacted with the diagram, using deictic gestures to refer to specific portions of the diagram during her explanation. Stacey also altered the diagram, adding new features as she began to consider them, then continued to reason while referencing the additions to her diagram. After a few repetitions of this behavior, Stacey drew an entirely new diagram (Figure 13, right), explaining the key idea that she would later use to construct her proof. Just before Stacey drew this second diagram, as she vocalized, "So if it wasn't..." she began speaking faster and with fewer pauses, seeming to suggest that she "got it." Stacey constructed a proof by contradiction, assuming that $A$ was not dense in $X$ and using this to confirm the existence of an open set disjoint from $A$.

Note the difference in the physical properties of the two diagrams (Figure 13, left and Figure 13, right) Stacey produced in this session. In particular, we emphasize the distance placed between the border of $A$ and the border of $X$, as the relative proximity of these two borders 
seemed to be a significant component of Stacey's understanding of what it means for a subset to be "dense." Furthermore, it was this change in distance between the borders of A and X that allowed Stacey to draw an open set that $\operatorname{did}$ not intersect $A$.

Stacey's task in Session 7 was to prove the following:

Prove: Let $(X, \mathcal{T})$ be a topological space. A separation of $X$ is a pair $U, V$ of disjoint open subsets of $X$ whose union is $X . X$ is connected if no separation of $X$ exists. If the sets $C, D$ form a separation of $X$ and if $Y$ is a connected subspace of $X$, then either $Y \subseteq C$ or $Y \subseteq D$.

When presenting this task, the facilitator accidentally excluded from the definition of separation the requirement that the sets $U$ and $V$ forming the separation must be nonempty. This did not seem to affect Stacey’s proving process.

Once again, she began by drawing a diagram (Figure 14, left).
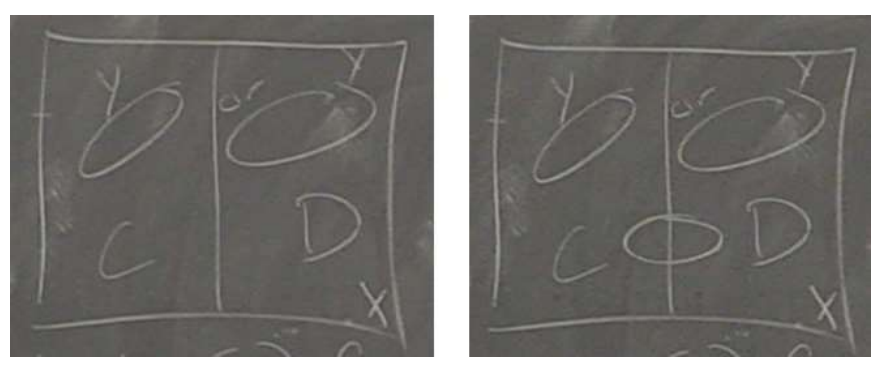

Figure 14: Stacey's diagram in Session 7.

Upon completing her diagram, Stacey explained,

I can tell you what I'm doing. If you have $X$, the ambient space [static point to the boundary of $X$ (Figure 14, left)], and then you have the sets $C$ and $D$ [alternating static points to the left and right rectangles (left)], they form a separation, so that means that they're disjoint [static points to the left rectangle, then the right (left)], so they don't have any of the same elements, and that their union is $X$ [static point 
to the boundary of $X(\mathrm{left})$ ], so that is satisfied for this [alternating static points to left and right rectangles (left)]. And then if $Y$ is connected, which means it's not in these sets [iconic gesture using thumb and index finger of each hand in adjacent U-shapes, indicating two disjoint subsets of $Y$ (Figure 15, left)] that are disjoint whose union is $Y$, it's just one cohesive set [iconic gesture cupping both hands into a $\mathrm{C}$-shape and putting them together to form a ball, indicating $Y$ as a connected set (Figure 15, right)], then it has to be either in $C$ or in $D$. It can't be in both, because if it was like that [draws the subset in Figure 14 (right)], it would be disjoint. [recognizes key idea]

Based on her reasoning, we believe that Stacey misspoke at the end of this quote, meaning to suggest that $Y$ would be disconnected, rather than disjoint.
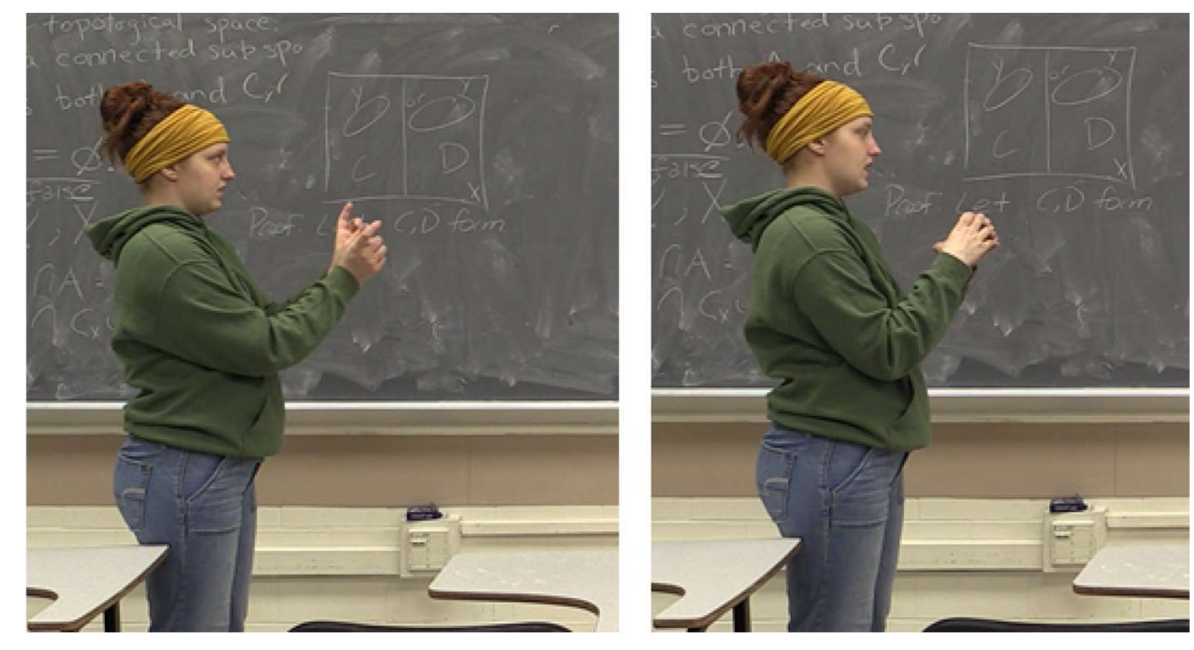

Figure 15: Stacey's metaphoric gestures representing a separation (left) and a connected space (right).

Stacey's initial diagram seemed to be a visual representation of her concept image (Tall \& Vinner, 1981) for this problem, constructed from her initial reading. Her explanation appeared to be for the benefit of the facilitator, rather than "thinking out loud" as it seemed to be in Session 5. Stacey's explanations were once again accompanied by gestures indicating components of the diagram, such as the disjoint open sets that together made up the separation. 
She also produced metaphorical gestures representing abstract properties of the mathematical objects in the conjecture, like the connectedness of the subspace $Y$. After Stacey produced metaphorical gestures referring to a separation of $Y$ and the connectedness of $Y$, she made one final addition to her diagram, leading to her expression of the key idea of the proof. She then wrote a correct proof of the statement by way of contradiction, using the key idea she identified from her diagram: she supposed that $Y$ intersected both $C$ and $D$, and derived a contradiction by finding a separation of $Y$.

Observe the physical aspects of Stacey's diagram: a connected space is one that can be drawn without a dividing line splitting it into two regions, and the existence of a separation corresponds to the existence of such a splitting line. With this imagery, a connected set that sits atop the dividing line of a separation immediately becomes disconnected, as it is physically separated into two disjoint regions by the existing boundary. This constituted the essence of Stacey's proof by contradiction.

In Session 9, Stacey was asked to prove the following:

Prove: If $Y$ is a compact subspace of the Hausdorff space $X$, and $x_{0}$ is a point of $X$ that is not in $Y$, then there exist disjoint open sets $U$ and $V$ containing $x_{0}$ and $Y$, respectively.

Although Stacey never identified the key idea of the proof in Session 9, the interplay of diagram modification and gesture guided her away from at least one inappropriate idea, and she continued to use these tools in further exploration of the conjecture.

Stacey began by reading the given statement and extracting information from the problem into a list written using mathematical notation (Figure 16), explaining that she "wrote it out in symbols to help [her] think of it better." It seemed that Stacey did not initially have a heuristic 
idea to convince herself of the truth of the claim in this session. In other sessions, Stacey quickly began to speak, draw, or gesture after she had read the prompt, giving some sort of intuitive description of the scenario. In this case, after Stacey finished writing down her list of information, she picked up her textbook and began to read. Shortly thereafter, she examined the problem again and reasoned that $Y$ must be closed, as it was a compact subspace of a Hausdorff space.

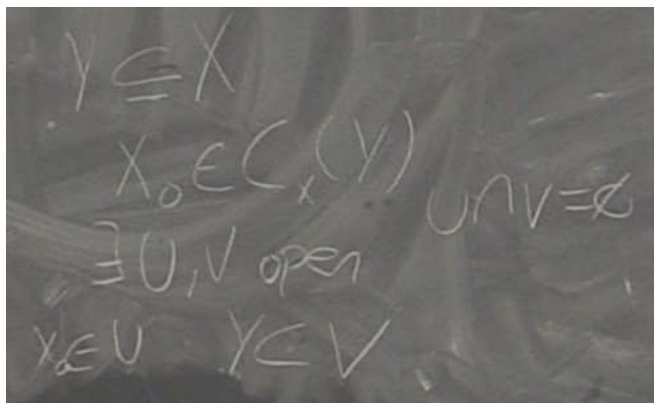

Figure 16: Stacey's notation extracted from the prompt in Session 9.

Upon making this realization, Stacey drew the diagram in Figure 17 (left).
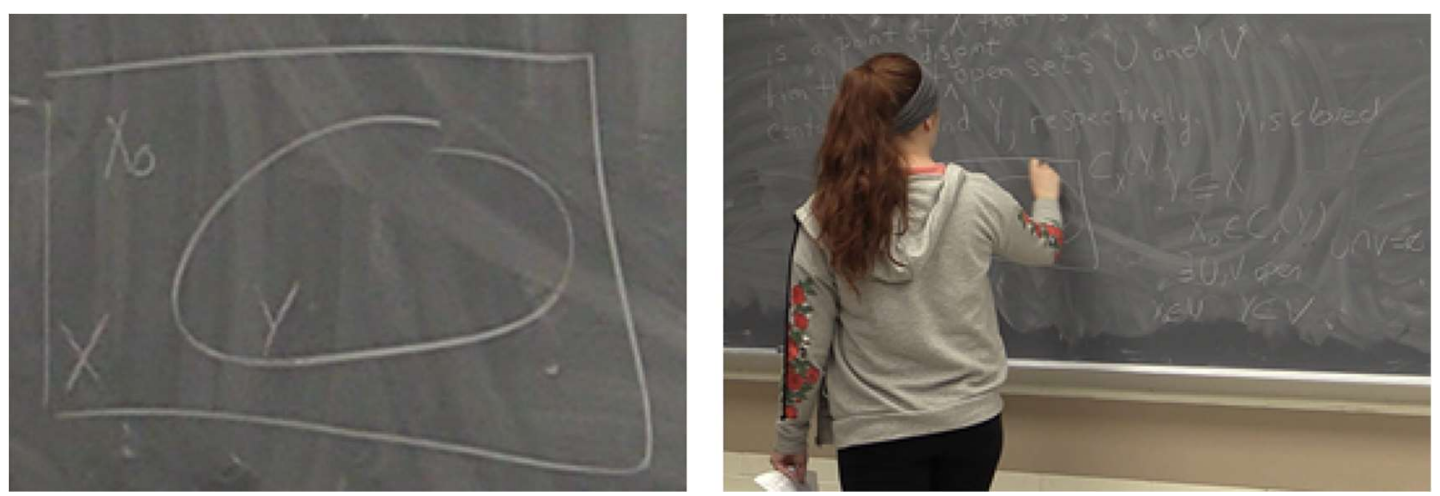

Figure 17: Stacey's diagram from Session 9.

Stacey explained,

The open set $U$ would be the complement of $Y$ in $X$ [writing $C_{X}(Y)$ (Figure 17, right)], because you know it's open because $Y$ is closed. I don't know if there's a theorem or something that would get me there, but the fact that $Y$ is a compact subspace of something Hausdorff might mean that there exists an open set within 
it [metaphoric gesture indicating a subset of $Y$ ] - oh wait, no. Because $Y$ has to be in $V$ [static point to her inscription, $Y \subseteq V$ (right)]. So we need something like this [draws a larger set containing $Y$, labels it $V$ (right)]. And then you also need their intersection to be empty. So I guess you can't really take just the complement of $Y$ [static point to the label $C_{X}(Y)$ (right)] because - you can't just take the set $Y$ because it's closed, you need an open set for $V$. So you need something bigger than $Y$ [tracing point to the boundary of $V$ (right)], but if you take anything bigger than $Y$, then you're in the complement of it [static point to the label $X$ ], and then the intersection isn't empty. So you need something within this complement of $Y$ [static point to $\left.C_{X}(Y)\right]$ to be the set $U$.

Based on the way she began this excerpt, it appeared that Stacey thought she had identified the key idea, but she was thinking about finding a $V$ such that $V \subseteq Y$ instead of $Y \subseteq V$. Nevertheless, Stacey's diagram construction appeared to be motivated by the fact that she thought she had a viable idea, or part of one, for how to prove the statement, based on her initial choice of $U=$ $C_{X}(Y)$. Upon consultation with the notation she had extracted from the problem, Stacey recognized the flaw in her selection. She was able to articulate the error in her reasoning using her diagram, explaining that $U=C_{X}(Y)$ was not the set she needed because that would force $V=Y$ in order to prevent $U$ and $V$ from intersecting. Beyond simply recognizing that her initial idea was flawed, Stacey's line of reasoning helped her refine her idea by showing that she needed a subset of $C_{X}(Y)$ to serve as the set $U$, since $V$ would need to contain $Y$.

Stacey continued to work on this problem for the entirety of Session 9, and she never gave any evidence that she had identified the key idea, nor did she begin to write a formal proof. But she continued in this way, using a combination of verbal communication, gestures, and 
diagram modification to reason about the problem, refining her reasoning through trial-and-error along the way. The rest of her work on this problem consisted of explorations of the various properties mentioned in the conjecture and their implications.

\section{Discussion}

The data presented suggest that Stacey's diagram construction was prompted by her perception that she had identified a suitable heuristic idea, that is, a general idea why the given statement was true (Raman, 2003). Once Stacey determined such an idea, she drew a diagram to represent the scenario in the problem and reasoned about the problem by means of a combination of speech, gestures, and alterations to her diagram. The interplay of these three aspects of her reasoning process led Stacey to recognize the key idea of the proof, which allowed her to then determine the procedural idea by translating her heuristic idea into logical-symbolic statements.

The conversion of the problem into a diagram necessarily imbued the abstract mathematical objects in Stacey's mind with physical and spatial properties that had direct correlations to mathematical properties in Stacey's mind (e.g., overlapping "blobs" indicated a nonempty intersection of sets, concentric blobs indicated set containment, etc.). Reasoning about her diagrams with gestures and manipulation of these physical properties in Sessions 5 and 7 led Stacey to recognize the key ideas of those proofs, that is, the ideas that gave her a sense of understanding of why the given statement was true and would subsequently manifest in her formal proofs. Stacey never identified a key idea in Session 9, as evidenced by the fact that she never attempted to begin writing a formal proof. However, we propose that she was following the same cognitive trajectory she followed in Sessions 5 and 7. Her diagram did not appear until she believed she had a suitable heuristic idea from which to work, and her diagram and gestures 
were produced in an effort to identify the key idea, but she simply did not reach this stage, hence she never produced a written proof.

\section{The Proof Writing Process}

Stacey's proving behaviors in Sessions 5, 7, and 9 followed the scheme shown in Figure 18 below.

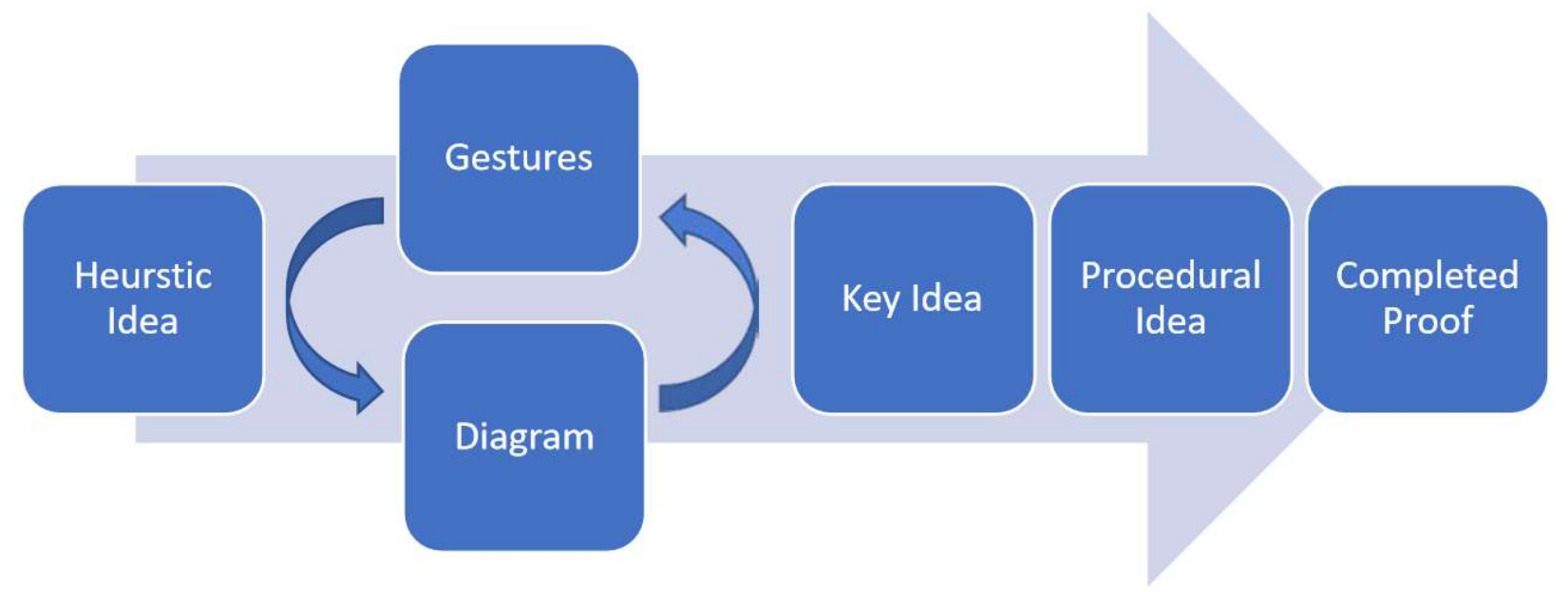

Figure 18: Stacey's proving trajectory in Sessions 5, 7, and 9.

The starting point in each of Sessions 5, 7, and 9 seemed to be the identification of a heuristic idea. Stacey began immediately constructing diagrams in Sessions 5 and 7 after she read the prompt. She explained her diagrams and how they represented the details from the problem, demonstrating that she had some kind of mental picture from which to work. In Session 9, Stacey did not immediately begin drawing; rather, she began by writing down a list of the information given in the prompt and consulting her textbook for clues. Upon realizing that the subspace Y was closed, hence its complement was open, Stacey began drawing her diagram and reasoning that $U=C_{X}(Y)$ was a reasonable choice for one of the required open sets. Although Stacey quickly recognized that this choice was inappropriate in general, her supposition that it might have been a viable choice appeared to trigger the beginning of her diagram construction. 
Stacey's explanation of her diagrams seemed to be the driving force behind her recognition of the key idea in Sessions 5 and 7. Although she had a heuristic idea in each case, it was not clear that she knew the same idea should work as a formal proof at first. In fact, it seemed that Stacey only recognized that her idea might work as a proof when she considered the physical limitations of her diagram. For example, in Session 5, Stacey seemed to convince herself that a set $A$ with the property that $A \cap O \neq \varnothing$ for each open set $O$ in $X$ must be dense in $X$ by drawing a diagram. The idea that became her formal proof, however, was the idea she discovered when the density of $\mathrm{A}$ in $\mathrm{X}$ was violated: that her diagram had the physical property that two "blobs" might not overlap. She engaged in this sort of reasoning again in Session 7 when she violated the given property that $Y$ must be connected by allowing it to sit atop the shared boundary of her separation. Stacey did not identify the key idea in Session 9, but in her effort to discover the key idea, she repeatedly altered her diagram and, through diagram alteration and gesture use, reasoned about the conjecture via physical properties she assigned to the objects in the problem.

Stacey continued to reason about each problem with gesture and diagram use until she recognized the key idea of the proof. We took Stacey's willingness to write a proof as evidence that she had discovered the key idea. As Raman (2003) defines it, the key idea is "an heuristic idea which one can map to a formal proof with appropriate sense of rigor" (p. 323). We do not assume that Stacey should know how to map this heuristic idea to a formal proof, only that it should. Thus, when Stacey moved on from her diagrammatic and gestural reasoning to begin writing a formal proof representing this reasoning, we took this to mean that she had ascertained that this heuristic idea could be transformed into a formal proof. 
Once Stacey had identified the key idea in Sessions 5 and 7, she immediately began the process of writing a formal proof. As Stacey was a novice prover, this process was not effortless in either case, and she occasionally required confirmation of the accuracy of her logic, language, and notation. In Session 9, Stacey never made an attempt to write a formal proof, but we note that she never identified the key idea of the proof in this session, thus preventing her from moving forward in her proving scheme.

\section{The Role of Embodied Cognition in Stacey's Proving Process}

In the sessions detailed in the data above, Stacey never (externally) referenced a specific example, nor did she appear to consider formal concept definitions, except to inform her diagram construction. Once a diagram had been constructed, Stacey's reasoning hinged upon the physical features of the diagram. For example, in Session 5, Stacey drew a diagram to represent $A$ as a dense subset of a topological space $X$. She explained that she wanted to draw the border of $A$ closer to the border of $X$, but she couldn't, suggesting that the physical proximity of the borders of the two sets was significant to her thought process. This detail is more significant than it may seem on the surface, as, topologically speaking, there need not even be a metric with which to measure distance in a given topology. Yet distance was a significant component of Stacey's concept image, as increasing the physical distance between the borders of $A$ and $X$ in order to fit an open set between them was central to the key idea Stacey developed into her proof in this session.

Stacey's diagram construction, gesture, and language appeared to attribute several physical properties to the mathematical objects under investigation in her reasoning. Stacey's conception of a dense subset seemed to rely heavily on the Near-Far schema (Lakoff, 2012). The boundary of the set $A$ and the boundary of $X$ were ascribed a very close physical proximity, and 
the assumption was made that taking the closure of $A$ meant extending the boundary of $A$ just a little bit more until it coincided with the boundary of $X$. The gap between the boundaries of $A$ and $X$ was assumed to be so small that it would be impossible for any open set - which were imbued with the property of being an enclosed region with some nonzero area - to exist in the gap. This is consistent with Stacey's language during other sessions in which she described finding the closure of a set as including all the points "arbitrarily close." Stacey's recognition of the key idea appeared to hinge on this notion of distance and arbitrary closeness; it was her consideration of what might happen if the distance between the boundaries of $A$ and $X$ were large enough to accommodate an open set that ultimately led her to recognize that this line of reasoning would translate into a proof (the key idea).

When reasoning about connected spaces and separations, Stacey's reasoning reflected the Container schema, in that her concept image of a connected set seemed to be a container with one continuous boundary and no subdivisions, while a disconnected set was represented by two adjacent containers sharing a common boundary. This reasoning made Stacey's contradiction obvious: If $Y$ wasn't contained entirely in $C$ or in $D$, then it would have to straddle the boundary separating $C$ and $D$, splitting $Y$ itself into two adjacent containers sharing a common boundary.

These structural components were embodied for Stacey, not simply in that she gestured about them, but because her gestures and her diagram suggested that these abstract mathematical objects had real, physical properties. Though a separation is defined simply in terms of open sets, which in general can take many forms, Stacey has imbued a separation with the physical representation of two containers which share a common boundary. Similarly, Stacey's gestures suggested that she viewed a connected set as a single container with no dividing boundaries. The contrast created by this assignment of physical properties to abstract entities appears to have 
prompted Stacey's recognition of the key idea. As her modification of the diagram at the end of the above excerpt shows, if a container with no internal boundary were to sit on top of such a boundary, then it would necessarily be split into two adjacent containers separated by a boundary - the connected set would be disconnected.

Once Stacey came to this realization, she immediately began to write a proof by way of contradiction, supposing $Y$ was connected and intersected both $C$ and $D$, and eventually finding a separation of $Y$. The idea that "It can't be in both, because if it was like that, it would be [disconnected]" became the key idea for Stacey: the idea seemed to convince her that the statement was true, and she recognized, through the use of her gestures and diagrams, that this idea would translate well into a proof.

Stacey constructed her diagram in Session 9 in response to an inappropriate heuristic idea. Stacey's inscription of notation extracted from the problem statement appeared to save her from this pitfall: her expectation that $V$ should be contained in $Y$ conflicted with the notation that stated $Y$ must be contained in $V$. This may have triggered conflicting mental images, causing Stacey to realize her error. Stacey's view of the set $V$ as a physical extension of the region contained within $Y$ and her perception of the overlap between $V$ and $C_{X}(Y)$ to be representative of a nonempty intersection made it clear that her initial choice of $U=C_{X}(Y)$ was insufficient to prove the given statement. Stacey's embodied view of these sets is evident in her word choice, as she expressed that she would need "something bigger than $Y$," but that taking something bigger than $Y$ would mean "being in the complement of it," her pointing gestures suggesting that she was referring to the complement of $Y$ as a physical location in the diagram. 


\section{The Role of Commognition in Stacey's Proving Process}

Sfard's $(2001,2009)$ theory of commognition regards thinking and communicating as two sides of the same coin. While the word communication typically refers specifically to interpersonal communication, thinking is regarded as intrapersonal communication, i.e., communication with oneself. Further, the rejection of the dualism of thinking and communication allows us to recognize deeper connections between the two:

The disappearance of a thinking-communicating dichotomy automatically eliminates several other splits. Indeed, once talking and gesturing stop being but "expressions" of thinking and become the process of thinking in itself, there is no point any longer in trying to separate the form (a sequence of words or body movements) from the content (thoughts, ideas) of communication, and it becomes equally pointless to speak about meanings "conveyed" in words, implying that it is a self-sustained entity of sorts, capable of appearing in many different verbal disguises. (Sfard, 2009, p. 195)

From this perspective, we view Stacey's gestures not simply as an expression of ideas she already holds in their final forms, but rather as the development of ideas.

Though Sfard (2009) referred to gesture and speech as two inseparable parts of commognition, she did propose different functions for what she calls "utterances" and gestures. Utterances provide humanity with the capacity for recursivity - the ability to "speak about speaking, to think about thinking” (p. 196). Recursivity allows for increases in complexity by condensing complicated ideas into signifiers which act as "repositories of complexity" (p. 197), encapsulations of complex hierarchies of ideas into compact notation. These repositories of complexity allow us to communicate more easily about complex information. Gesture, according 
to Sfard, serves the purpose of "realizing mathematical signifiers" (2009, p. 198). That is, gestures serve the purpose of clarifying what is meant by the utterances being used, and they help ensure that both the speaker and the listener are referencing the same objects and features with their terminology. This can be done using deictic gestures, in the case that the referent is present, or using iconic or representational gestures in the case that the referent is not physically present.

Stacey, for her part, seemed to use her diagrams as repositories of complexity. The prompts given in the sessions contained complex information, some of them using terminology with which Stacey was not yet familiar. Each proof required the use of multiple pieces of information working in concert to produce a logical argument. As such, prior to constructing her proofs, Stacey extracted information from each prompt and deposited that information into a diagram. Stacey was then able to talk and gesture more easily about the given information, forming connections and making hypothesis, each new connection being added to the repository through a modification of the diagram. As each addition was made to the diagram, Stacey was then able to make further connections and see how all the pieces of information given worked together to form the basis of the proof she would later construct. In this way, her diagrams served as a sort of storage facility for mathematical information, allowing her to add new information one or two pieces at a time until she had all the information she needed in one place to form her argument.

Stacey's gestures were not only for the benefit of interpersonal communication; they also helped her keep her own thinking on track. In Session 9, we saw that Stacey initially constructed her diagram while thinking about an incorrect heuristic idea: she was thinking about finding an open subset contained in $Y$, rather than finding an open subset that contained $Y$. She only 
recognized that she was moving down the wrong path when she pointed to her inscription that read $Y \subseteq V$. This effort to communicate her idea verbally, supported with gestures, exposed the error in her reasoning when a conflict arose between her utterances and her gestures, and it allowed her to recalibrate and approach the problem along a different path.

\section{Conclusions}

In this paper, we addressed the following research question: "How do students synergize cognition, gestures, diagrams, and communication when writing proofs in topology?" We presented a case study of Stacey, an undergraduate taking a first course in topology. When working on a proof, Stacey based her diagram on a heuristic idea representing her understanding of the problem, then used a combination of speech, gestures, and diagram modification to arrive at the key idea of the proof, which she then used to write a formal proof.

We argued that Stacey's diagrams and her gesture use grounded the abstract mathematical ideas Stacey was working with, allowing her to reason about them in terms of the physical properties that her diagrams and gestures imbued them with. We also argued, in line with Sfard's (2009) theory of commognition, that Stacey's speech and gestures were not just elements of her self-expression, but they were integral components of her thinking, and gave her a physical means of transforming her understanding over the course of working on a proof.

The embodied perspective suggests that gestures and speech occur together spontaneously, and the brain uses conceptual schema to reason about abstract ideas in terms of physical properties. This, together with the notion that gesture and speech are components of cognition, suggest that Stacey's external communication of her ideas - whether she was talking to herself or to somebody else - played a vital role in Stacey's evolving concept image of the problems she worked on. The combination of Stacey's verbal communication, gesture, and 
diagrams also appeared to prevent Stacey from spending time working on unproductive proving strategies.

This work adds to a growing body of literature suggesting that gestures and verbal communication support student thinking about and understanding of mathematics. An interesting future project would be the investigation of this kind of reasoning in other branches of mathematics, such as modern algebra or differential equations. Topology is intimately connected with physical notions like distance and connectivity; whether this kind of visual/embodied reasoning is useful in other fields of mathematics would be of great interest to the pedagogical community. Even if this approach is not applicable across different fields, learning how to translate verbal-symbolic information into a diagrammatic form and interpret it from an appropriate embodied perspective may present a powerful content-specific tool for students writing proofs in topology. 


\section{SUMMARY OF PAPERS}

In the papers presented above, I described an in-depth case study of Stacey, an undergraduate taking a first course in introductory topology. I examined Stacey's proving behaviors from three different perspectives: problem solving, example use, and embodied cognition and commognition. In each paper, I described the contribution made by the relevant perspective to Stacey's recognition of the key idea of the proof (Raman, 2003) and to her subsequent writing of a proof.

In Paper 1, I examined Stacey's behaviors through the lens of the Problem-Solving Cycle of Carlson and Bloom's (2005) Multidimensional Problem-Solving Framework. Assuming that proving and problem solving were similar in their cognitive demands, my intent was to determine the extent to which Stacey's mathematical behaviors mirrored those of expert mathematicians. I observed that Stacey did progress through essentially the same series of phases when writing her proofs that experts do when solving problems, but with differences in the order in which she engaged in each phase. With respect to the checking phase, Stacey also frequently conducted external checking of her progress by asking questions of the mathematical authority present during her proof writing.

Prior to data collection, I expected that Stacey might rely heavily on examples when working with concepts in topology due to her relative inexperience in advanced mathematics. In Paper 2, I sought to answer the research questions "What kinds of examples do students use to help them write proofs in topology, and how do they use them?" I identified a type of abstract visual representation that Stacey reasoned about as if it were an example, hence I named objects of this type structural examples. Structural examples possess all the essential properties of a definition or a given situation (e.g., a mathematical conjecture), but no other properties. Stacey 
used her structural examples to gain an intuitive sense of the statement she was trying to prove or disprove, to gain insight into the technique for proving a true statement by manipulating the properties of the example, and to identify the properties of the necessary counterexample when disproving false statements.

Paper 2 made it clear that Stacey's structural examples played a pivotal role for her in the identification of key ideas, but a more thorough analysis of how Stacey's structural examples led her to recognize key ideas was warranted. I noted that Stacey frequently gestured when talking about her structural examples. This, coupled with her manipulations of the spatial properties of her examples during the planning phase of Carlson and Bloom's framework, led to the analysis in Paper 3. Paper 3 described Stacey's proving behaviors through the combined lenses of embodied cognition (Lakoff \& Núñez, 2000) and commognition (Sfard, 2009). From this perspective, I identified that Stacey used speech, gestures, and diagrams as part of her thinking, rather than as representations of her thinking. Furthermore, Stacey's representation of mathematical objects using structural examples grounded those objects in the physical world, assigning them the physical properties that Stacey manipulated in her search for key ideas.

\section{CONNECTIONS AND CONCLUSIONS}

The results of Papers 1, 2, and 3 combine to form an in-depth view of Stacey's cognitive construction of the elements of mathematical structure necessary to complete proofs in topology (Chihara, 2004; Piaget, 1985; Resnik, 1981; Shapiro, 1997; von Glasersfeld, 2005; Wadsworth, 1975). From a top-down view, Stacey's development of the concepts of topological structures was viewed using Carlson and Bloom's (2005) Multidimensional Problem-Solving Framework. Recall that Carlson and Bloom's Problem-Solving Cycle consists of four main phases: orienting, planning, executing, and checking. During each phase of this cycle, Stacey engaged in particular 
behaviors tailored toward uncovering the necessary structures to complete her proofs. In the following, I detail how Stacey's behaviors in each phase aided in her cognitive construction of the relevant topological structures and the roles played by her structural examples and her interactions with them as grounded representations of her mental constructs.

Carlson and Bloom's original results remark that, among other behaviors, all of the expert mathematicians in their study were "remarkably efficient in constructing either a picture or a mental image of the problem situation as they attempted to make sense of the question" (Carlson \& Bloom, 2005, p. 62) during the orienting phase of the Problem-Solving Cycle. My analysis of Stacey's behaviors in Paper 1 shows that Stacey often exhibited the same behavior, and furthermore, it identified the goal of this behavior as the recognition of the key idea of the proof (Raman, 2003). This result supports work by Stylianou (2002) which argued that mathematicians construct visual representations of problem scenarios in problem solving for the deliberate purpose of extracting information from them, but extends the scope of the result to the writing of formal proofs. The discussion in Paper 3 highlights the role of Stacey's visual representations as embodiments of a heuristic idea held in Stacey's mind, and Paper 2 further recognizes Stacey's visual representations as structural examples, which she will later manipulate in her quest for the key idea. The recognition of Stacey's visual representations as examples suggests that, for Stacey, there exists an injective mapping of the specific properties of her example onto the abstract structures of the mathematical object she holds in her mind. Thus, manipulations of her structural examples translated to manipulations of the mathematical objects they were created to represent.

The planning phase of Carlson and Bloom's Problem-Solving Cycle contained a subcycle they referred to as conjecture-imagine-evaluate. While they reported that this process 
largely took place entirely within their subjects' minds, Stacey demonstrated this process externally through the manipulations of her structural examples. Stacey's structural examples not only acted as instantiations of her concept image, but through their embodiment as physical entities (diagrams), Stacey imbued her examples with spatial properties. The grounding of Stacey's mental images in the form of diagrams allowed for the manipulation of their physical properties, leading to implied consequences on the basis of the cognitive schema associated with the structure of the diagram. The diagram's role as an example then allowed Stacey to internalize the manipulations she performed and map them from their embodied properties back onto their abstract mathematical properties. The interplay of Stacey's speech and gestures about her structural examples and the spatial properties those examples inherited as embodied representations of abstract mental structures allowed Stacey to physically manipulate abstract mental objects. This ultimately led to the achievement of Stacey's implicit goal of identifying the key ideas of her proofs.

The executing phase of Carlson and Bloom's framework was the phase in which their experts produced their mathematical arguments after they had identified a suitable strategy. This corresponds to the assembly of the procedural idea (Raman, 2003) into a formal proof. Stacey's executing phase consisted of the translation of the spatial properties of her structural examples into formal mathematical symbols and terminology. In the cases when Stacey was disproving false statements, her executing activities consisted of the identification and construction of an appropriate counterexample. During the course of Stacey's formal proof writing, she often returned to the planning phase to reexamine some of the ideas she had expressed there. Stacey was inexperienced with the intuition of topology as well as the notation and language used in 
topology proofs. Thus, Stacey often transitioned briefly into the checking phase as well to ensure that her logic, notation, and language were appropriate.

Stacey's checking behaviors were primarily external, while those of the experts in Carlson and Bloom's study were largely internal. Because Stacey had identified what she believed to be the key idea, her checking primarily consisted of asking questions about notation and language. Often, she wrote down what she believed to be appropriate notation to express her ideas, then she consulted with me, expressing her intentions in plain English and asking if her mathematical language appropriately conveyed her argument. At the end of our sessions, we frequently holistically reviewed Stacey's progress for the purpose of her edification, as I had intended for these sessions to benefit the student participants as well.

\section{CONTRIBUTIONS TO THE LITERATURE AND IMPLICATIONS FOR TEACHING}

Topology is routinely taught as proof-based courses at the undergraduate and graduate levels of mathematics. Most notably, this study contributes to the literature on the teaching and learning of undergraduate topology, which is sparse, and of advanced mathematics as a whole. Furthermore, proof writing and interpretation are notoriously challenging skills for students to master at all levels. My work provides new perspectives on students' reasoning about proofs and demonstrates a trajectory that turned out to be not only effective for proof writing, but instructive for my participant's understanding of concepts in topology.

Although this is an isolated case study of one student's behaviors, my results provide useful insights into productive student behaviors. First, this result provides support for the pedagogical suggestion that students should produce visual arguments on which to base their formal mathematics (Fiorella \& Mayer, 2016; Raman, 2003; Weber \& Alcock, 2004; D. Zazkis et al., 2016; R. Zazkis et al., 1996). Moreover, students should be instructed and encouraged to 
use their visual representations in exploration of concepts (Booth \& Thomas, 2000; Stylianou, 2002; R. Zazkis et al., 1996). Stacey’s success in writing proofs was not a consequence of simply having a visual representation; she used her visual representations as aids in her search for key ideas. I agree with Leron $(1983,1985)$ that, rather than presenting students with proofs written in their final form, teachers should present proofs by first providing an intuitive idea of what the proof will show and then filling in details later. The intuitive idea Leron described is exactly the key idea described by Raman, and the discovery of this idea was precisely the primary goal of Stacey's proof writing process in my data. Showing proofs in their final form obscures much of the intuition behind the arguments presented and perpetuates the idea that mathematicians are effortlessly brilliant and discover these complicated proofs in their final form. I believe this sort of proof presentation also encourages the memorization of proofs rather than their discovery, creating a culture of "mathematics as memorization" instead of "mathematics as discovery." Getting students to see, as Stacey did, that looking for an intuitive explanation and then working to formalize it may encourage their success in proof writing. From a teacher's perspective, I believe that the creative portion of mathematics that involves devising ideas and recognizing why they work is the most challenging to instill in students. Once they "get the idea," the rest is often mechanical.

Finally, my work provides support for the employment of the embodied cognition perspective and the theory of commognition in the advanced mathematics classroom. Though they are often taught in a very abstract way through definitions and theorems, mathematical ideas are rooted in intuition about structure and the physical world. Encouraging students to view mathematics as an entity grounded in physical and spatial reasoning and to leverage the corresponding embodied properties in their reasoning can lead to keen insights about abstract 
ideas. Furthermore, with the recognition that talking and gesturing are inherently part of the process of thinking, we should encourage students to reason aloud, to draw diagrams, and to gesture while thinking about mathematics, even if they are only talking to themselves. The process of communicating ideas $i s$ the process of reasoning about those ideas; to inhibit oneself from speaking, drawing, and gesturing is to inhibit oneself from taking full advantage of their cognitive faculties.

\section{FUTURE RESEARCH}

Topology as a whole remains largely unstudied by the undergraduate mathematics education research community. Studies to investigate what areas of topology (content, pedagogy, or otherwise) are the most problematic for teachers and students would greatly benefit the research community. Specific future studies should investigate students' concept images of topics in topology, their relevance to concept definitions, and their usefulness in constructing proofs. Studies are also needed to investigate students' development and uses of explicit examples (see Paper 2) in topology. I am also curious to learn about students' conceptions of the field of topology as a whole, i.e., what the field studies and its significance to mathematics. I suspect there may be a range of responses including geometry and spatial relationships and applications to analysis, among others. Students' perceptions of the field may influence their approaches to problems, perhaps prompting some students to reason geometrically, others to reason through examples from analysis, and others to reason simply by manipulation of symbols and definitions.

With regard to structural examples, the prevalence of this phenomenon should be studied not only in topology but in other fields of advanced mathematics like abstract algebra and differential equations as well. The geometric nature of topology may push students to reason in 
terms of visual structure more so in this field than in others. Studies may also investigate the particular topics in topology for which visual strategies prove most useful for students. Teaching experiments may also be useful to identify instructional strategies to help students use structural examples and visual representations in more productive manners.

It is well established that interpersonal communication of mathematical ideas can support student learning. Studies should also examine students' self-explanatory behaviors, specifically in the context of proof. My results suggest that reasoning aloud, even if only for one's own benefit, may transform students' thinking about a proof and support their recognition of the key idea.

Finally, though there is already some work in this area (Carlson \& Bloom, 2005; Stylianou, 2002), I believe the mathematics education community would benefit from more studies on the proving and problem-solving behaviors of expert mathematicians and graduate students. Studies in undergraduate mathematics education tend to focus on undergraduates and the obstacles they face in learning. Observation of the behaviors of experts may provide useful information about what behaviors we might want our students to develop, and examination of graduate students could provide some insight into productive learning trajectories leading to those results. Studies of the development of mathematical ideas over time could lead to invaluable insights about the kinds of activities in which lower-level students should be engaged. 


\section{BIBLIOGRAPHY}

Alcock, L. (2004). Uses of Example Objects in Proving. In M. J. Hoines \& A. B. Fuglestad (Eds.), Proceedings of the 28th Conference of the International Group for the Psychology of Mathematics Education (Vol. 2, pp. 17-24). Bergen, Norway.

Alcock, L., \& Inglis, M. (2008). Doctoral Students' Use of Examples in Evaluating and Proving Conjectures. Educational Studies in Mathematics, 69(2), 111-129. doi:10.1007/s10649008-9149-x

Alcock, L., \& Weber, K. (2010). Undergraduates' Example Use in Proof Construction: Purposes and Effectiveness. Investigations in Mathematics Learning, 3(1), 1-22. doi:10.1080/24727466.2010.11790298

Alibali, M. W., \& Nathan, M. J. (2007). Teachers' Gestures as a Means of Scaffolding Students' Understanding: Evidence from an Early Algebra Lesson. In R. Goldman, R. Pea, B. Barron, \& S. J. Derry (Eds.), Video Research in the Learning Sciences. New York, NY: Routledge.

Alibali, M. W., Nathan, M. J., Wolfgram, M. S., Breckenridge Church, R., Jacobs, S. A., Johnson Martinez, C., \& Knuth, E. J. (2014). How Teachers link ideas in mathematics instruction using speech and gesture: a corpus analysis. Cognition and Instruction, 32(1), 65-100. doi:10.1080/07370008.2013.858161

Alibali, M. W., Spencer, R. C., Knox, L., \& Kita, S. (2011). Spontaneous Gestures Influence Strategy Choices in Problem Solving. Psychological Science, 22(9), 1138-1144. doi:10.1177/0956797611417722

Anderson, J. R. (1978). Arguments Concerning Representations for Mental Imagery. Psychological Review, 85(4), 249-277.

Arzarello, F., Paola, D., Robutti, O., \& Sabena, C. (2009). Gestures as Semiotic Resources in the Mathematics Classroom. Educational Studies in Mathematics, 70, 97-109. doi:10.1007/s10649-008-9163-z

Azrou, N., \& Khelladi, A. (2019). Why Do Students Write Poor Proof Texts? A Case Study on Undergraduates' Proof Writing. Educational Studies in Mathematics, 102, 257-274. doi:https://doi.org/10.1007/s10649-019-09911-9

Barsalou, L. W. (1999). Perceptual Symbol Systems. Behavioral and Brain Sciences, 22, $577-$ 660.

Barsalou, L. W. (2008). Grounded Cognition. The Annual Review of Psychology, 59, 617-645. doi:10.1146/annurev.psych.59.103006.093639

Beilock, S. L., \& Goldin-Meadow, S. (2010). Gesture Changes Thought by Grounding It in Action. Psychological Science, 21(11), 1605-1610.

Berger, A., \& Stewart, S. (2018). Schema Development in an Introductory Topology Proof. Paper presented at the The 21st Annual Conference on Research in Undergraduate Mathematics Education, San Diego, CA.

Berger, A., \& Stewart, S. (2019). Analyzing Topology Students' Schema Qualities. Paper presented at the The 22nd Annual Conference on Research in Undergraduate Mathematics Education, Oklahoma City, OK.

Bernard, J. A., Millman, Z. B., \& Mittal, V. A. (2015). Beat and Metaphoric Gestures are Differentially Associated With Regional Cerebellar and Cortical Volumes. Human Brain Mapping, 36, 4016-4030. 
Bills, L., \& Tall, D. (1998). Operable Definitions in Advanced Mathematics: The Case of the Least Upper Bound. Paper presented at the 22nd Conference of the International Group for the Psychology of Mathematics Education, Stellenbosch, South Africa.

Booth, R. D. L., \& Thomas, M. O. J. (2000). Visualization in Mathematics Learning: Arithmetic Problem-solving and Student Difficulties. Journal of Mathematical Behavior, 18(2), 169190.

Braun, V., \& Clarke, V. (2006). Using thematic analysis in psychology. Qualitative Research in Psychology, 3(2), 77-101. doi:10.1191/1478088706qp063oa

Carlson, M. P., \& Bloom, I. (2005). The Cyclic Nature of Problem Solving: An Emergent Multidimensional Problem-Solving Framework. Educational Studies in Mathematics, $58(1), 45-75$.

Cheshire, D. C. (2017). ON THE AXIOMATIZATION OF MATHEMATICAL UNDERSTANDING: CONTINUOUS FUNCTIONS IN THE TRANSITION TO TOPOLOGY. (Doctor of Philosophy), Texas State University, Ann Arbor, MI. ProQuest database. (10737124)

Chihara, C. S. (2004). A Structural Account of Mathematics. New York, NY: Oxford University Press.

Clements, K. (1982). Visual Imagery and School Mathematics. For the Learning of Mathematics, 2(3), 33-39.

Cohen, L., Manion, L., \& Morrison, K. (2013). Research Methods in Education (7th ed.): Routledge.

Comi, A., \& Eppler, M. J. (2011, September 7-9, 2011). Visual Representations As Carriers and Symbols of Organizational Knowledge. Paper presented at the 11th International Conference on Knowledge Management and Knowledge Technologies, Graz, Austria.

Dargue, N., Sweller, N., \& Jones, M. P. (2019). When Our Hands Help Us Understand: A MetaAnalysis Into the Effects of Gesture on Comprehension. Psychological Bulletin, 145(8), 765-784.

Dawkins, P. C., \& Weber, K. (2016). Values and Norms of Proof for Mathematicians and Students. Educational Studies in Mathematics, 123-142.

Dimmel, J. K., \& Herbst, P. G. (2015). The Semiotic Structure of Geometry Diagrams: How Textbook Diagrams Convey Meaning. Journal for Research in Mathematics Education, 46(2), 147-195.

Dorier, J.-L., \& Sierpinska, A. (2001). Research Into the Teaching and Learning of Linear Algebra. In D. Holton, et al. (Ed.), The teaching and learning of mathematics of university level: an ICMI study. Dordrecht: Kluwer Academic Publishers.

Fiorella, L., \& Mayer, R. E. (2016). Eight Ways to Promote Generative Learning. Educational Psychology Review, 28, 717-741. doi:10.1007/s10648-015-9348-9

Fukawa-Connelly, T. P., \& Newton, C. (2014). Analyzing the Teaching of Advanced Mathematics Courses Via the Enacted Example Space. Educational Studies in Mathematics, 87, 323-349. doi:10.1007/s10649-014-9554-2

Gallagher, K., \& Engelke Infante, N. (2019). A Possible Framework for Students' Proving in Introductory Topology. Paper presented at the The 22nd Annual Conference on Research in Undergraduate Mathematics Education, Oklahoma City, OK.

Gemignani, M. C. (2015). Elementary Topology. New York: Dover Publications, Inc.

Gibson, D. (1998). Students' Use of Diagrams to Develop Proofs in an Introductory Analysis Course. Research in Collegiate Mathematics Education III, 7, 284-307. 
Glenberg, A. M., \& Robertson, D. A. (2000). Symbol Grounding and Meaning: A Comparison of High-Dimensional and Embodied Theories of Meaning. Journal of Memory and Language, 43, 379-401. doi:10.1006/jmla.2000.2714

Goldinger, S. D., Papesh, M. H., Barnhart, A. S., Hansen, W. A., \& Hout, M. C. (2016). The Poverty of Embodied Cognition. Psychonomic Bulletin \& Review, 23, 959-978. doi:10.3758/s13423-015-0860-1

Gray, E., \& Tall, D. (2007). Abstraction as a Natural Process of Mental Compression. Mathematics Education Research Journal, 19(2), 23-40.

Hamilton, L., \& Corbett-Whittier, C. (2014). Using Case Study in Education Research. London: SAGE Publications Ltd.

Harel, G., \& Soto, O. (2017). Structural Reasoning. International Journal of Research in Undergraduate Mathematics Education, 3, 225-242. doi:10.1007/s40753-016-0041-2

Harel, G., \& Sowder, L. (1998). Students' Proof Schemes: Results from Exploratory Studies. In A. H. Schoenfeld, J. Kaput, \& E. Dubinsky (Eds.), Research in Collegiate Mathematics Education. III (Vol. 7, pp. 234-283): American Mathematical Society.

Harel, G., \& Tall, D. (1991). The General, the Abstract, and the Generic in Advanced Mathematics. For the Learning of Mathematics, 11(1), 38-42.

Hostetter, A. B., \& Alibali, M. W. (2008). Visible Embodiment: Gestures as Simulated Action. Psychonomic Bulletin \& Review, 15(3), 495-514. doi:10.3758/PBR.15.3.495

Iannone, P., \& Inglis, M. (2010). Self efficacy and mathematical proof: are undergraduate students good at assessing their own proof production ability? Paper presented at the 13th Annual Conference on Research in Undergraduate Mathematics Education, Raleigh, NC.

Kendon, A. (2004). Gesture: Visible Action As Utterance: Cambridge University Press.

Komatsu, K., Jones, K., Ikeda, T., \& Narazaki, A. (2017). Proof Validation and Modification in Secondary School Geometry. Journal of Mathematical Behavior, 47, 1-15.

Lakoff, G. (2012). Explaining Embodied Cognition Results. Topics in Cognitive Science, 4(4), 773-785. doi:10.1111/j.1756-8765.2012.01222.x

Lakoff, G., \& Núñez, R. E. (2000). Where Mathematics Comes From: How the Embodied Mind Brings Mathematics Into Being. New York, NY: Basic Books.

Leron, U. (1983). Structuring Mathematical Proofs. The American Mathematical Monthly, 90(3), 174-185.

Leron, U. (1985). Heuristic Presentations: The Role of Structuring. For the Learning of Mathematics, 5(3), 7-13.

Lester, F. K., Jr. (1994). Musings About Mathematical Problem-Solving Research: 1970-1994. Journal for Research in Mathematics Education, 25(6), 660-675.

Marton, F., \& Tsui, A. B. M. (2004). Classroom Discourse and the Space of Learning. Mahwah, NJ: Lawrence Erlbaum Associates.

Mason, J. (1992). Doing and Construing Mathematics in Screenspace. Paper presented at the 15th Annual Conference of the Mathematics Education Research Group of Australasia, Sydney, Australia.

Mason, J., \& Pimm, D. (1984). Generic Examples: Seeing the General in the Particular. Educational Studies in Mathematics, 15(3), 277-289.

Mason, J., Stephens, M., \& Watson, A. (2009). Appreciating Mathematical Structure for All. Mathematics Education Research Journal, 21(2), 10-32. 
Mason, J., \& Watson, A. (2008). Mathematics as a Constructive Activity: Exploiting Dimensions of Possible Variation. In M. P. Carlson \& C. Rasmussen (Eds.), Making the Connection: Research and Teaching in Undergraduate Mathematics Education (pp. 191-204): Mathematical Association of America.

McNeill, D. (1992). Hand and Mind: What gestures reveal about thought. Chicago, IL: The University of Chicago Press.

McNeill, D. (2005). Gesture \& Thought. Chicago, IL: University of Chicago Press.

Mendelson, B. (2012). Introduction to Topology (Third ed.): Courier Corporation.

Mills, M. (2014). A Framework for Example Usage in Proof Presentations. Journal of Mathematical Behavior, 33, 106-118.

Moore, R. C. (1994). Making the Transition to Formal Proof. Educational Studies in Mathematics, 27(3), 249-266.

Narayanan, S. (1997). Embodiment in Language Understanding: Sensory-Motor Representations for Metaphoric Reasoning about Event Descriptions. University of California at Berkeley,

Parsons, C. (1995). Structuralism and the Concept of Set. In W. Sinnott-Armstrong, D. Raffman, \& N. Asher (Eds.), Modality, Morality, and Belief: Essays in Honors of Ruth Barcan Marcus (pp. 14-92). Cambridge, England: Cambridge University Press.

Piaget, J. (1985). The Equilibration of Cognitive Structures (T. B. a. K. J. Thampy, Trans.). Chicago and London: The University of Chicago Press.

Pinto, M., \& Tall, D. (2002). Building Formal Mathematics on Visual Imagery: A Case Study and a Theory. For the Learning of Mathematics, 22(1), 2-10.

Pólya, G. (1957). How to Solve It (2nd ed.): Princeton University Press.

Presmeg, N. C. (1986). Visualization and Mathematical Giftedness. Educational Studies in Mathematics, 17(3), 297-311.

Presmeg, N. C. (1992). Prototypes, Metaphors, Metonymies and Imaginative Rationality in High School. Educational Studies in Mathematics, 23(6), 595-610.

Radford, L. (2009). Why do gestures matter? Sensuous cognition and palpability of mathematical meanings. Educational Studies in Mathematics, 70, 111-126. doi:10.1007/s10649-008-9127-3

Raman, M. (2001). Beliefs About Proof in Collegiate Calculus. Paper presented at the The 23rd Annual Meeting of the International Group for the Psychology of Mathematics Education, Snowbird, Utah.

Raman, M. (2003). Key Ideas: What Are They And How Can They Help Us Understand How People View Proof? Educational Studies in Mathematics, 52, 319-325.

Rasmussen, C., Stephan, M., \& Allen, K. (2004). Classroom Mathematical Practices and Gesturing. Journal of Mathematical Behavior, 23, 301-323. doi:10.1016/j.jmathb.2004.06.003

Resnik, M. D. (1981). Mathematics as a Science of Patterns: Ontology and Reference. Nôus, 15(4), 529-550.

Roth, W.-M. (2000). From gesture to scientific language. Journal of Pragmatics, 32(11), 16831714. doi:10.1016/S0378-2166(99)00115-0

Roth, W.-M. (2001). Gestures: Their Role in Teaching and Learning. Review of Educational Research, 71(3), 365-392. 
Selden, A., \& Selden, J. (2003). Validations of Proofs Considered as Texts: Can Undergraduates Tell Whether an Argument Proves a Theorem? Journal for Research in Mathematics Education, 34(1), 4-36.

Sfard, A. (2001). There Is More to Discourse Than Meets the Ears: Looking at Thinking as Communicating to Learn More about Mathematical Learning. Educational Studies in Mathematics, 46, 13-57.

Sfard, A. (2009). What's all the fuss about gestures? A commentary. Educational Studies in Mathematics, 70, 191-200. doi:10.1007/s10649-008-9161-1

Shapiro, S. (1997). Philosophy of Mathematics: Structure and Ontology. Oxford: Oxford University Press.

Shoenfeld, A. H. (1982). Some Thoughts on Problem-Solving and Mathematics Education. In F. K. Lester, Jr. \& J. Garofalo (Eds.), Mathematical Problem Solving: Issues in Research (pp. 27-37). Philadelpha: Franklin Institute Press.

Shoenfeld, A. H. (1983). The Wild, Wild, Wild, Wild, Wild World of Problem Solving (A Review of Sorts). For the Learning of Mathematics, 3, 40-47.

Sinclair, N., Watson, A., Zazkis, R., \& Mason, J. (2011). The Structuring of Personal Example Spaces. The Journal of Mathematical Behavior, 30(4), 291-303. doi:10.1016/j.jmathb.2011.04.001

Stewart, J. (2016). Calculus: Early Transcendentals (Eighth ed.). Boston, MA: Cengage Learning.

Straube, B., Green, A., Bromberger, B., \& Kircher, T. (2011). The Differentiation of Iconic and Metaphoric Gestures: Common and Unique Integration Processes. Human Brain Mapping, 32, 520-533.

Stylianou, D. A. (2002). On The Interaction of Visualization and Analysis: The Negotiation of a Visual Representation in Expert Problem Solving. Journal of Mathematical Behavior, 21, 303-317.

Tall, D. (2008). The Transition to Formal Thinking in Mathematics. Mathematics Education Research Journal, 20(2), 5-24.

Tall, D., \& Vinner, S. (1981). Concept Image and Concept Definition in Mathematics with Particular Reference to Limits. Educational Studies in Mathematics, 12(2), 151-169.

Varela, F. J., Rosch, E., \& Thompson, E. (1993). The Embodied Mind: Cognitive Science and Human Experience (1st ed.). Cambridge, MA: The MIT Press.

Vinner, S. (2002). The role of definitions in the teaching and learning of mathematics. In D. O. Tall (Ed.), Advanced Mathematical Thinking (pp. 65-81). Dordrecht, The Netherlands: Kluwer.

von Glasersfeld, E. (2005). Learning and Adaptation in the Theory of Constructivism. In L. Smith (Ed.), Critical Readings on Piaget (pp. 20-27). New York, NY: Routledge.

Wadsworth, B. J. (1975). Piaget's Theory of Cognitive Development: An Introduction for Students of Psychology and Education. New York: David McKay Company, Inc.

Watson, A., \& Mason, J. (2005). Mathematics as a Constructive Activity: Learners Generating Examples. Mahwah, New Jersey: Lawrence Erlbaum Associates.

Weber, K. (2001). Student Difficulty In Constructing Proofs: The Need For Strategic Knowledge. Educational Studies in Mathematics, 48, 101-119.

Weber, K. (2009). How Syntactic Reasoners Can Develop Understanding, Evaluate Conjectures, and Generate Counterexamples in Advanced Mathematics. Journal of Mathematical Behavior, 28(2-3), 200-208. doi:10.1016/j.jmathb.2009.08.001 
Weber, K., \& Alcock, L. (2004). Semantic and Syntactic Proof Productions. Educational Studies in Mathematics, 56(2/3), 209-234.

Weber, K., \& Alcock, L. (2009). Semantic and Syntactic Reasoning and Proving in Advanced Mathematics Classrooms. In M. Blanton, D. Stylianaiou, \& E. Knuth (Eds.), The Teaching and Learning of Proof Across the K-16 Curriculum. New York: Routledge.

Wilson, M. (2002). Six Views of Embodied Cognition. Psychometric Bulletin \& Review, 9(4), 625-636.

Yin, R. K. (2003a). Applications of Case Study Research (2nd ed. Vol. 34). Thousand Oaks, CA: SAGE Publications.

Yin, R. K. (2003b). Case Study Research: Design and Methods (3rd ed. Vol. 5). Thousand Oaks, California: SAGE Publications.

Zandieh, M., Roh, K. H., \& Knapp, J. (2011, October 20-23, 2011). Using Conceptual Blending to Analyze Student Proving. Paper presented at the 33rd Annual Conference of the North American Chapter of the International Group for the Psychology of Mathematics Education, Reno, NV.

Zazkis, D., Weber, K., \& Mejía-Ramos, J. P. (2016). Bridging the Gap Between Graphical Arguments and Verbal-Symbolic Proofs in a Real Analysis Context. Educational Studies in Mathematics, 93, 155-173. doi:10.1007/s10649-016-9698-3

Zazkis, R., Dubinsky, E., \& Dautermann, J. (1996). Coordinating Visual and Analytic Strategies: A Study of Students' Understanding of the Group D4. Journal for Research in Mathematics Education, 27(4), 435-457.

Zazkis, R., \& Leikin, R. (2007). Generating Examples: From Pedagogical Tool to a Research Tool. For the Learning of Mathematics, 27(2), 15-21.

Zazkis, R., \& Leikin, R. (2008). Exemplifying Definitions: A Case of a Square. Educational Studies in Mathematics, 69(2), 131-148. doi:10.1007/s10649-008-9131-7

Zhen, B., Weber, K., \& Mejia-Ramos, J. P. (2016). Mathematics Majors' Perceptions of the Admissibility of Graphical Inferences in Proofs. International Journal of Research in Undergraduate Mathematics Education, 2, 1-29. doi:10.1007/s40753-015-0010-1 


\section{APPENDIX A: SESSION 5 (PROVE) CODED FOR PAPER 1}

The transcript excerpt below is the Prove task from Session 5, as coded for Paper 1.

Speaker identifiers were abbreviated in the SPEAKER column: I is the Facilitator (Interviewer),

$\mathrm{S}$ is Stacey, and $\mathrm{T}$ is Tom.

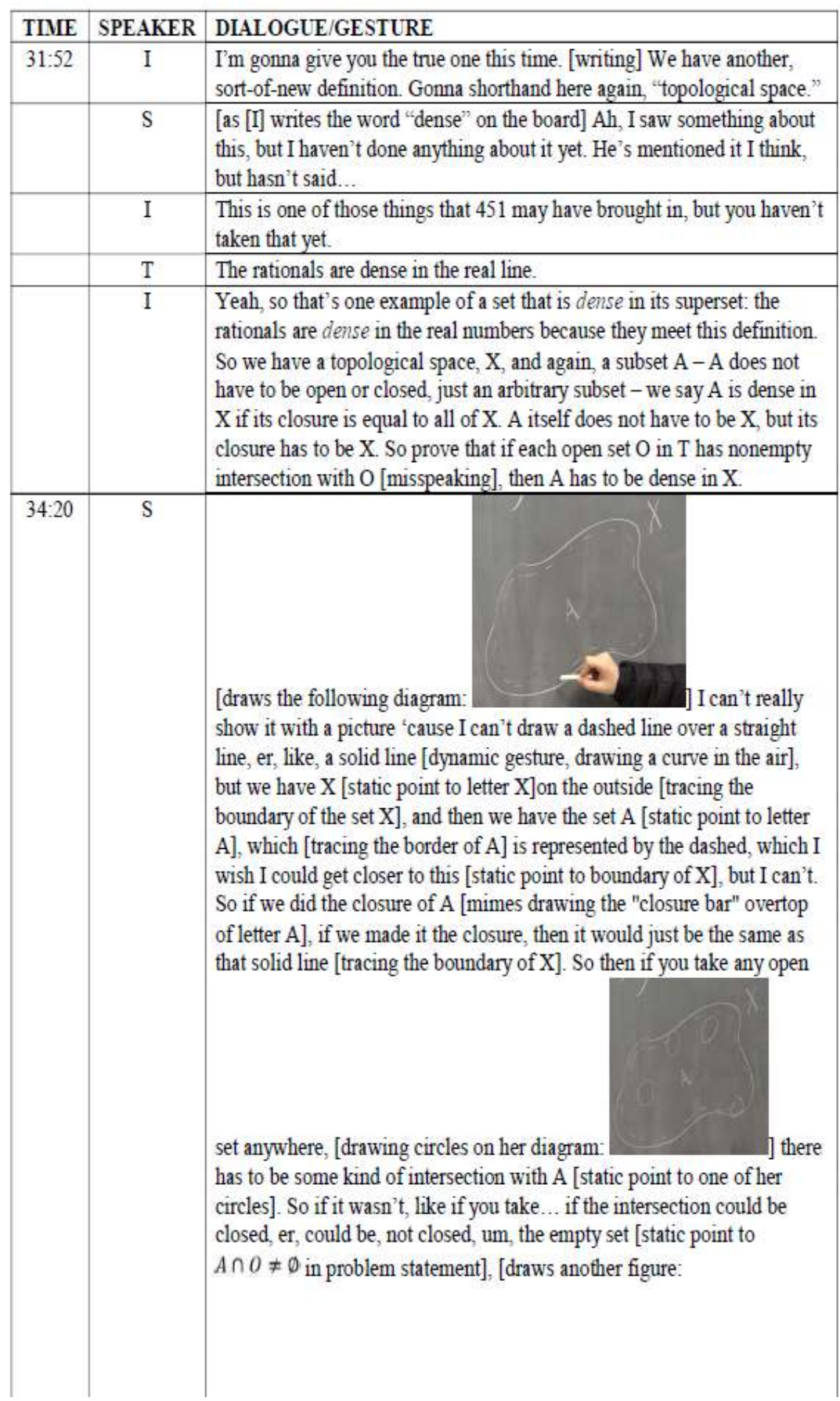




\begin{tabular}{|c|c|c|}
\hline & & $\begin{array}{l}\text { ] you've got X here and A here... you could have } \\
\text { an open set here, and their intersection would be the empty set, but then } \\
\text { this closure [static point to boundary of A in new diagram] wouldn't be } \\
\text { equal to X [static point to the boundary of X]. So I get it conceptually I } \\
\text { think, but I'm not sure how to prove it. What are you thinking, [T]? [long } \\
\text { pause, picking up textbook] So we probably have to use the definition of } \\
\text { closure in it... [pointing to something in her textbook] }\end{array}$ \\
\hline \multirow[t]{10}{*}{$37: 19$} & $\mathrm{~T}$ & Yeah. \\
\hline & $\mathrm{S}$ & $\begin{array}{l}\text { So we could say, like... take } \mathrm{x} \text { in... one of either A or X, I'm not sure } \\
\text { which one yet... and then a neighborhood of that point } x . . .\end{array}$ \\
\hline & I & $\begin{array}{l}\text { Is there maybe a general strategy that you're thinking about? How are } \\
\text { you thinking about approaching this problem? }\end{array}$ \\
\hline & $\mathrm{S}$ & Um, I think contradiction, is kind of what's in my head right now. \\
\hline & I & $\begin{array}{l}\text { OK, so if you had to outline your procedure - I know you don't have the } \\
\text { whole thing fleshed out - but how would your contradiction look? How } \\
\text { would you set that up? }\end{array}$ \\
\hline & $\mathrm{S}$ & $\begin{array}{l}\text { [pause, looks at problem statement for } 5 \text { seconds, then at diagrams for } 7 \\
\text { seconds, then again briefly before she writes] Uh... some... [writing, but } \\
\text { then erases what she wrote] }\end{array}$ \\
\hline & $\mathrm{T}$ & $\begin{array}{l}\text { So for the contradiction for this statement [static point at the problem } \\
\text { statement], it's gonna be "For each open set } O \text { [static point to " } O \in \mathcal{T} "] \text {, } \\
\text { we have this, [pointing to " } A \cap O \neq \emptyset \text { "] but } A \text { is not dense in X[tracing } \\
\text { point along "A is not dense in } X " \text { as he reads aloud]. So the closure of } A \\
\text { is not X. [static point to " } \bar{A}=X " \text { in problem statement] Right? }\end{array}$ \\
\hline & $\mathrm{S}$ & OK. Yeah. \\
\hline & I & $\begin{array}{l}\text { So if you were gonna start writing a proof, what would the first line of } \\
\text { your proof look like? }\end{array}$ \\
\hline & $\mathrm{S}$ & $\begin{array}{l}\text { I don't know, we have to pick like, a point, within either A or X, but I'm } \\
\text { not sure which one to pick it from... I think it would be A. So pick a } \\
\text { point, like a in A, [squinting at [I], possibly looking for confirmation] is } \\
\text { what I think [unintelligible]... }\end{array}$ \\
\hline \multirow[t]{2}{*}{ 39:57 } & I & $\begin{array}{l}\text { Take a look again at the definition of closure and see if that gets you } \\
\text { anywhere. }\end{array}$ \\
\hline & $\mathrm{S}$ & $\begin{array}{l}\text { [pause while looking at textbook, then looking between problem and } \\
\text { textbook] I don't know why, but I'm having trouble like, reconciling it } \\
\text { between having A and X and open sets and closures all [unintelligible] } \\
\text { able to put all those in the right order. }\end{array}$ \\
\hline
\end{tabular}




\begin{tabular}{|c|c|c|}
\hline & I & $\begin{array}{l}\text { Well, you were saying you're having trouble deciding whether you want } \\
\text { to pick a point from } \mathrm{A} \text { or pick a point from } \mathrm{X} \text {. }\end{array}$ \\
\hline & $\mathrm{S}$ & Yeah. \\
\hline & $\mathrm{I}$ & Does the definition of closure help you make a decision? \\
\hline & $\mathrm{S}$ & [reading from her textbook] A point $\mathrm{x}$ is said to be in the closure of A... \\
\hline & $\mathrm{T}$ & So you'd have to pick a point in $\mathrm{X}$. \\
\hline & I & $\begin{array}{l}\text { Why would you not pick a point in A? Why would that not really be very } \\
\text { helpful? }\end{array}$ \\
\hline \multirow[t]{17}{*}{$41: 36$} & $\mathrm{~T}$ & Because you're trying to prove that the closure of $\mathrm{A}$ is not the whole $\mathrm{X}$. \\
\hline & I & Well, you're assuming that the closure of $\mathrm{A}$ is not the whole $\mathrm{X}$. \\
\hline & $\mathrm{T}$ & Oh yeah. \\
\hline & $\mathrm{I}$ & If you're doing a proof by contradiction. \\
\hline & $\mathrm{S}$ & $\begin{array}{l}\text { Oh, OK So we're picking a point in } \mathrm{X} \text { and showing that it's not in the } \\
\text { closure of A. [looking at [I], possibly for confirmation] That's what we } \\
\text { wanna try to... }\end{array}$ \\
\hline & $\mathrm{T}$ & No, we assume there's a point $x$ that's not gonna be in the closure... \\
\hline & $\mathrm{S}$ & That isn't, OK, we're assuming that whole thing. \\
\hline & $\mathrm{T}$ & Yeah. [looks at [I]] \\
\hline & $\mathrm{I}$ & Does that make sense why that's your assumption? \\
\hline & $\mathrm{S}$ & $\begin{array}{l}\text { Because then [static point to } " A \cap X \neq \emptyset n \text { in the problem statement] their } \\
\text { intersection would be the null set... [looking at [I]] }\end{array}$ \\
\hline & $\mathrm{I}$ & Well, so that's gonna be something you wanna show. \\
\hline & $\mathrm{S}$ & Yeah. \\
\hline & $\mathrm{I}$ & 'Cause you were talking about a proof by contradiction, right? \\
\hline & $\mathrm{S}$ & ( \\
\hline & I & $\begin{array}{l}\text { So before you even start doing any work, what's the assumption that } \\
\text { you're gonna make in your proof? That very first line of your proof, what } \\
\text { are you gonna assume to be happening in this problem that makes it a } \\
\text { proof by contradiction? }\end{array}$ \\
\hline & $\mathrm{T}$ & $\begin{array}{l}\text { So you're gonna assume there's a point in } \mathrm{X} \text { that's not in the closure of } \mathrm{A} \\
\text { [static point to } \bar{A}=X \text { ]. }\end{array}$ \\
\hline & $\mathrm{S}$ & And then that would mean that $\mathrm{A}$ is not dense in $\mathrm{X}$. \\
\hline \multirow[t]{5}{*}{$42: 53$} & I & $\begin{array}{l}\text { Right. Yeah, so when you do a proof by contradiction, you suppose the } \\
\text { hypothesis is true, right? So suppose that for each open set } \mathrm{O} \text { in } \mathrm{T} \text {, we } \\
\text { have that the intersection is nonempty, but then assume that } \mathrm{A} \text { is not } \\
\text { dense in X. So we assume that part is false. }\end{array}$ \\
\hline & $\mathrm{T}$ & Yeah. \\
\hline & $\mathrm{S}$ & $\begin{array}{l}\text { So then that's our, how we're assuming that, that we're letting } \mathrm{x} \text { be in } \mathrm{X} \\
\text { but not in } \mathrm{A} \text { ? }\end{array}$ \\
\hline & I & $\begin{array}{l}\text { So we know that the closure of } \mathrm{A} \text { has to be a subset of X, right? Because } \\
\text { everything in the closure is gonna be in the space. }\end{array}$ \\
\hline & $\mathrm{T}$ & Yeah. \\
\hline
\end{tabular}




\begin{tabular}{|c|c|c|}
\hline & $\mathrm{S}$ & So we're saying that... [writing "Let $x \in X$ and $x \notin \bar{A}$ "] Like that? \\
\hline & I & $\begin{array}{l}\text { And why can we do that? Why can we choose that } \mathrm{x} \text { ? How do we know } \\
\text { that one exists in this proof? }\end{array}$ \\
\hline & $\mathrm{T}$ & Because we tried to assume the... false... \\
\hline & $\mathrm{S}$ & The opposite. [points from up to down, indicating a switch] \\
\hline & $\mathrm{T}$ & The opposite. \\
\hline & I & The opposite of what? \\
\hline & $\mathrm{S}$ & This. [underlines "then $\mathrm{A}$ is dense in X."] \\
\hline & $\mathrm{T}$ & $\mathrm{A}$ is dense in $\mathrm{X}$. \\
\hline & I & $\begin{array}{l}\text { OK, so we're assuming } \mathrm{A} \text { is not dense in } \mathrm{X} \text {, so that's how we know that } \\
\text { that } \mathrm{x} \text { exists. [long pause] }\end{array}$ \\
\hline \multirow[t]{11}{*}{$44: 36$} & $\mathrm{~S}$ & 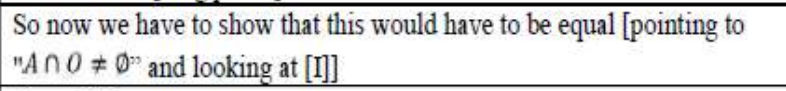 \\
\hline & I & So... OK. \\
\hline & $\mathrm{T}$ & 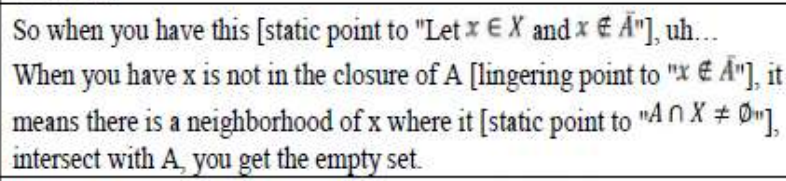 \\
\hline & $\mathrm{S}$ & 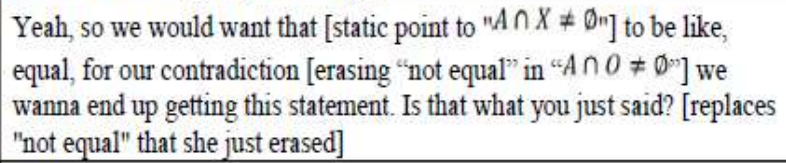 \\
\hline & $\mathrm{T}$ & Yeah. \\
\hline & $\mathrm{I}$ & I think he's telling you the next step in your proof. \\
\hline & $\mathrm{S}$ & OK You wanna just write it? \\
\hline & $\mathrm{T}$ & It doesn't seem right... \\
\hline & I & $\begin{array}{l}\text { Go ahead and write what you're thinking, and then we can assess it from } \\
\text { there. }\end{array}$ \\
\hline & $\mathrm{S}$ & $\begin{array}{l}\text { Yeah, I have some trouble thinking when people say things out loud } \\
\text { sometimes. }\end{array}$ \\
\hline & $\mathrm{T}$ & $\begin{array}{l}\text { [tracing point along "Let } x \in X \text { and } x \in \vec{A} \text {," writes " } \exists N \in \mathcal{N}_{x} \text { s.t. } \\
N \cap A=\emptyset \text { " then points to what he wrote while reading silently] So it, so } \\
\text { when we have this [static point to } N \cap A=\emptyset \text { ], it already contradicts with } \\
\text { this assumption. [static point to } O \in \mathcal{T} \text { ] }\end{array}$ \\
\hline \multirow[t]{6}{*}{ 46:07 } & $\mathrm{I}$ & How does that contradict? \\
\hline & $\mathrm{T}$ & $\begin{array}{l}\text { Because now you have an open set that, intersect with A, gives you, uh, } \\
\text { empty set. [static point either to } N \cap A=\emptyset \text { or to Stacey's first diagram] }\end{array}$ \\
\hline & $\mathrm{I}$ & Is $\mathrm{N}$ an open set? \\
\hline & $\mathrm{T}$ & No. It's a neighborhood. \\
\hline & $\mathrm{I}$ & It's a neighborhood? So where is the open set coming from. \\
\hline & $\mathrm{S}$ & It's within the neighborhood? [looking at [I]] \\
\hline
\end{tabular}

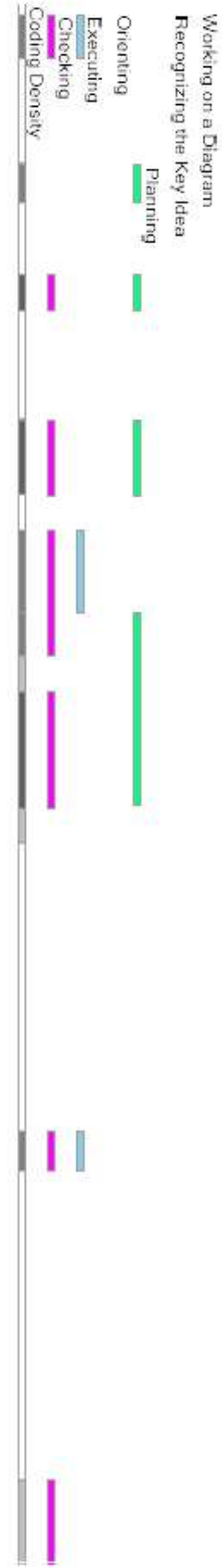




\begin{tabular}{|c|c|}
\hline $\mathrm{I}$ & OK. So because $\mathrm{N}$ is a neighborhood, it contains an open set. \\
\hline $\mathrm{T}$ & Yeah. \\
\hline $\mathrm{I}$ & $\mathrm{OK}$ \\
\hline $\mathrm{S}$ & $\begin{array}{l}\text { So there's } \mathrm{O} \text {, subset of } \mathrm{N} \text {, whose intersection with } \mathrm{A} \text { is equal to the } \\
\text { empty set. }\end{array}$ \\
\hline I & I agree with that. \\
\hline $\mathrm{T}$ & 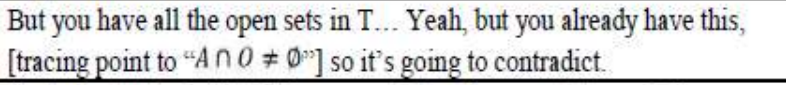 \\
\hline I & $\begin{array}{l}\text { Yeah. So that's it, right? We assumed that A was not dense in X, so } \\
\text { there's a neighborhood that has an empty intersection with A, so there } \\
\text { has to be an open set inside that neighborhood that has an empty } \\
\text { intersection with A. }\end{array}$ \\
\hline $\mathrm{T}$ & $\mathrm{Oh} !$ \\
\hline $\mathrm{I}$ & But we know that that can't happen. \\
\hline $\mathrm{S}$ & [writes "So $\left.\exists 0 \subset N_{\text {s.t. }} O \cap A=\emptyset "\right]$ \\
\hline $\mathrm{T}$ & $\begin{array}{l}\text { The only difficulty when I think about a neighborhood and an open set... } \\
\text { When you have this [pointing to "N } \cap A=\emptyset \text { "] and then you can say that } \\
\text { there's an open set that, intersect with A, gives you the empty } \\
\text { intersection. }\end{array}$ \\
\hline $\mathrm{I}$ & So making the jump from the neighborhood to the open set is difficult? \\
\hline $\mathrm{T}$ & $\begin{array}{l}\text { Yeah. Isn't the neighborhood [static point to } N \cap A=\emptyset \text { ] the collection of } \\
\text { open sets? [dynamic gesture, shaping his hands to trace the outline of a } \\
\text { ball in the air] }\end{array}$ \\
\hline $\mathrm{I}$ & It just contains an open set. \\
\hline $\mathrm{T}$ & It contains an open set. \\
\hline I & It doesn't necessarily have to be a union of them. \\
\hline I & $\begin{array}{l}\text { So if we have, let's say, what this is telling me is, [drawing] if this is my } \\
\text { set A, let's say that I have a neighborhood over here that's disjoint from } \\
\text { A. It's a neighborhood, so it contains an open set, let's call this Ox, and } \\
\text { put x in here... }\end{array}$ \\
\hline $\mathrm{S}$ & At least one, but it can contain more? \\
\hline I & $\begin{array}{l}\text { Right. It can contain as many as you want, but it has to have at least one } \\
\text { open set inside it. }\end{array}$ \\
\hline $\mathrm{T}$ & Yeah. \\
\hline I & $\begin{array}{l}\text { So if we have a neighborhood that's disjoint from A, the neighborhood } \\
\text { contains an open set... }\end{array}$ \\
\hline $\mathrm{T}$ & Oh! So... \\
\hline $\mathrm{I}$ & The open set's gonna be farther away from A, probably. \\
\hline $\mathrm{T}$ & Yeah. \\
\hline I & So that intersection definitely should be empty too. \\
\hline $\mathrm{T}$ & This is so obvious! \\
\hline $\mathrm{S}$ & $\begin{array}{l}\text { So then we just say, like if we're actually writing this proof out, we write } \\
\text { that line [tracing point along the last line of the proof on the board], and }\end{array}$ \\
\hline
\end{tabular}

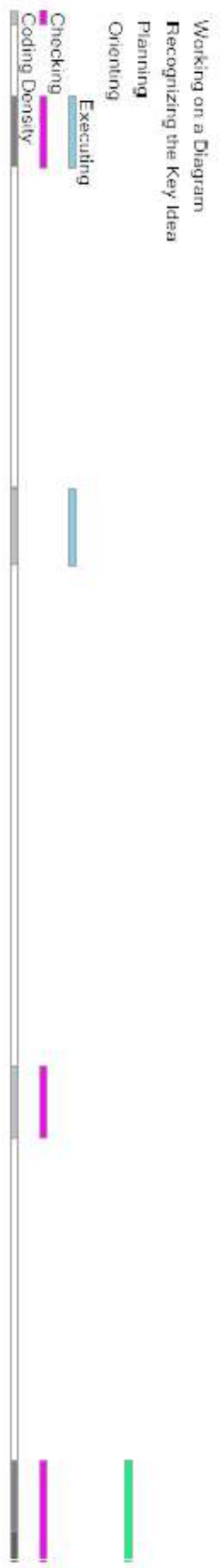


we say "Thus, by contradiction, this statement...

\begin{tabular}{|l|l|l}
\hline 49:00 & I & Mhmm. Yeah, so we found an open set whose intersection with $\mathrm{A}$ is
\end{tabular} empty, but by assumption, it's not, so we contradicted. 


\section{APPENDIX B: SESSION 5 (PROVE) CODED FOR PAPERS 2 AND 3}

The transcript excerpt below is the Prove task from Session 5, as coded for Papers 2 and 3.

Speaker identifiers were abbreviated in the SPEAKER column: I is the Facilitator (Interviewer),

$\mathrm{S}$ is Stacey, and $\mathrm{T}$ is Tom.

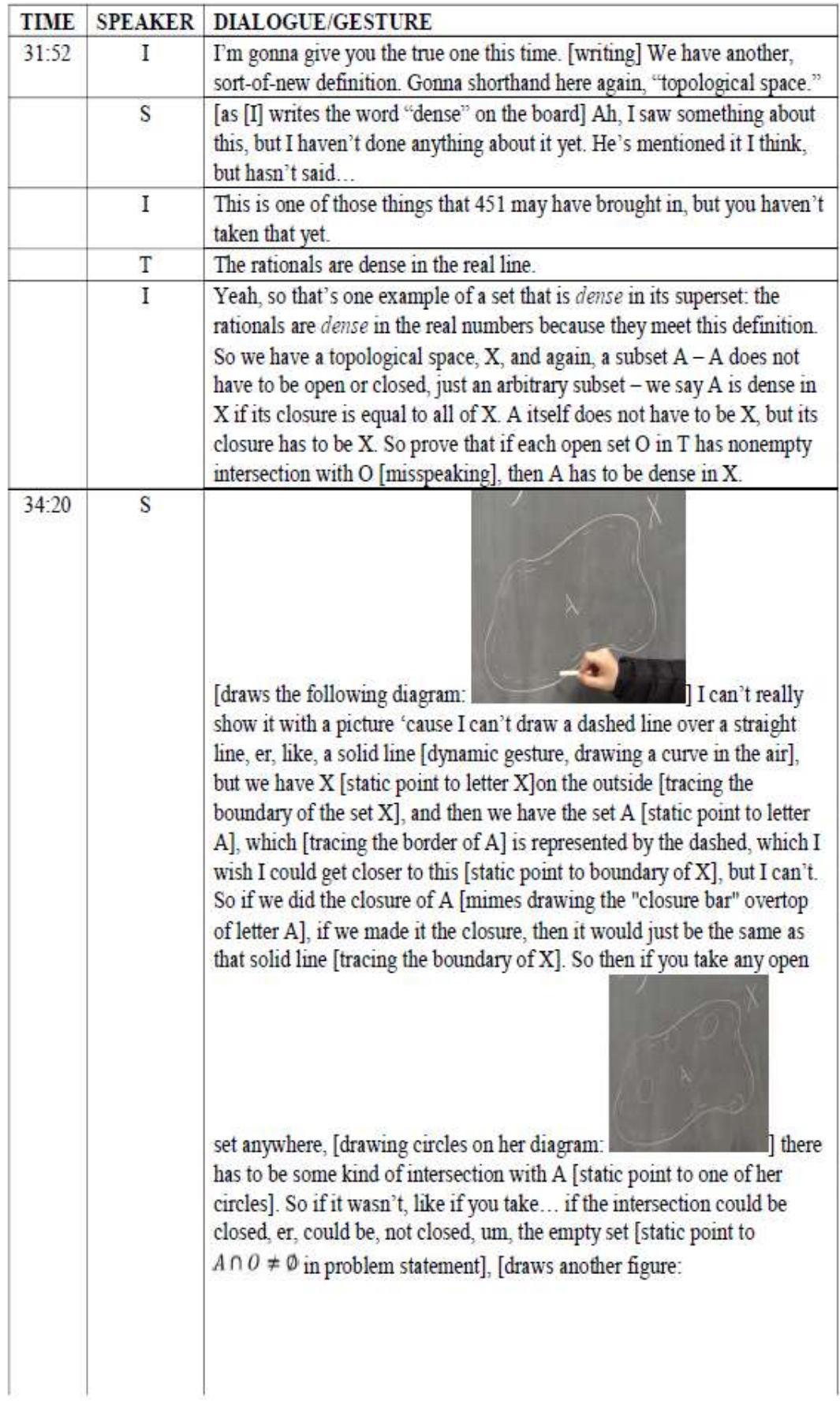

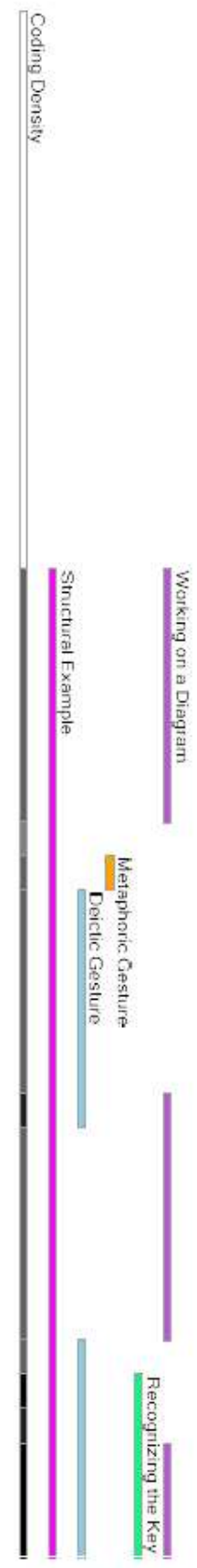




\begin{tabular}{|c|c|c|}
\hline & & $\begin{array}{l}\text { ] you've got X here and A here... you could have } \\
\text { an open set here, and their intersection would be the empty set, but then } \\
\text { this closure [static point to boundary of A in new diagram] wouldn't be } \\
\text { equal to X [static point to the boundary of X]. So I get it conceptually I } \\
\text { think, but I'm not sure how to prove it. What are you thinking, [T]? [long } \\
\text { pause, picking up textbook] So we probably have to use the definition of } \\
\text { closure in it... [pointing to something in her textbook] }\end{array}$ \\
\hline \multirow[t]{10}{*}{$37: 19$} & $\mathrm{~T}$ & Yeah. \\
\hline & $\mathrm{S}$ & $\begin{array}{l}\text { So we could say, like... take } \mathrm{x} \text { in... one of either } \mathrm{A} \text { or } \mathrm{X}, \mathrm{I} \text { 'm not sure } \\
\text { which one yet... and then a neighborhood of that point } \mathrm{x} . .\end{array}$ \\
\hline & I & $\begin{array}{l}\text { Is there maybe a general strategy that you're thinking about? How are } \\
\text { you thinking about approaching this problem? }\end{array}$ \\
\hline & $\mathrm{S}$ & Um, I think contradiction, is kind of what's in my head right now. \\
\hline & I & $\begin{array}{l}\text { OK, so if you had to outline your procedure - I know you don't have the } \\
\text { whole thing fleshed out - but how would your contradiction look? How } \\
\text { would you set that up? }\end{array}$ \\
\hline & $\mathrm{S}$ & $\begin{array}{l}\text { [pause, looks at problem statement for } 5 \text { seconds, then at diagrams for } 7 \\
\text { seconds, then again briefly before she writes] Uh... some... [writing, but } \\
\text { then erases what she wrote] }\end{array}$ \\
\hline & $\mathrm{T}$ & $\begin{array}{l}\text { So for the contradiction for this statement [static point at the problem } \\
\text { statement], it's gonna be "For each open set } O \text { [static point to " } O \in \mathcal{T} "] \text {, } \\
\text { we have this, [pointing to " } A \cap O \neq \emptyset " \text { ] but } A \text { is not dense in } X[\text { tracing } \\
\text { point along "A is not dense in } X " \text { as he reads aloud]. So the closure of A } \\
\text { is not X. [static point to " } \bar{A}=X \text { " in problem statement] Right? }\end{array}$ \\
\hline & $\mathrm{S}$ & OK. Yeah. \\
\hline & I & $\begin{array}{l}\text { So if you were gonna start writing a proof, what would the first line of } \\
\text { your proof look like? }\end{array}$ \\
\hline & $\mathrm{S}$ & $\begin{array}{l}\text { I don't know, we have to pick like, a point, within either A or X, but I'm } \\
\text { not sure which one to pick it from... I think it would be A. So pick a } \\
\text { point, like a in A, [squinting at [I], possibly looking for confirmation] is } \\
\text { what I think [unintelligible]... }\end{array}$ \\
\hline \multirow[t]{2}{*}{ 39:57 } & $\mathrm{I}$ & $\begin{array}{l}\text { Take a look again at the definition of closure and see if that gets you } \\
\text { anywhere. }\end{array}$ \\
\hline & $\mathrm{S}$ & $\begin{array}{l}\text { [pause while looking at textbook, then looking between problem and } \\
\text { textbook] I don't know why, but I'm having trouble like, reconciling it } \\
\text { between having A and X and open sets and closures all [unintelligible] } \\
\text { able to put all those in the right order. }\end{array}$ \\
\hline
\end{tabular}




\begin{tabular}{|c|c|c|}
\hline & I & $\begin{array}{l}\text { Well, you were saying you're having trouble deciding whether you want } \\
\text { to pick a point from A or pick a point from X. }\end{array}$ \\
\hline & $\mathrm{S}$ & Yeah. \\
\hline & I & Does the definition of closure help you make a decision? \\
\hline & $\mathrm{S}$ & [reading from her textbook] A point $\mathrm{x}$ is said to be in the closure of A... \\
\hline & $\mathrm{T}$ & So you'd have to pick a point in $\mathrm{X}$. \\
\hline & I & $\begin{array}{l}\text { Why would you not pick a point in A? Why would that not really be very } \\
\text { helpful? }\end{array}$ \\
\hline \multirow[t]{17}{*}{$41: 36$} & $\mathrm{~T}$ & Because you're trying to prove that the closure of $\mathrm{A}$ is not the whole $\mathrm{X}$. \\
\hline & I & Well, you're assuming that the closure of $\mathrm{A}$ is not the whole $\mathrm{X}$. \\
\hline & $\mathrm{T}$ & Oh yeah. \\
\hline & I & If you're doing a proof by contradiction. \\
\hline & $\mathrm{S}$ & $\begin{array}{l}\text { Oh, OK. So we're picking a point in } \mathrm{X} \text { and showing that it's not in the } \\
\text { closure of A. [looking at [I], possibly for confirmation] That's what we } \\
\text { wanna try to... }\end{array}$ \\
\hline & $\mathrm{T}$ & No, we assume there's a point $x$ that's not gonna be in the closure... \\
\hline & $\mathrm{S}$ & That isn't, OK, we're assuming that whole thing. \\
\hline & $\mathrm{T}$ & Yeah. [looks at [I]] \\
\hline & I & Does that make sense why that's your assumption? \\
\hline & $\mathrm{S}$ & 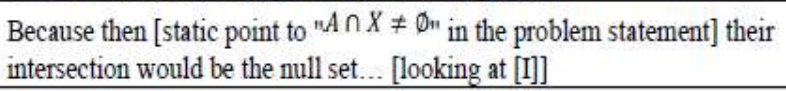 \\
\hline & I & Well, so that's gonna be something you wanna show. \\
\hline & $\mathrm{S}$ & Yeah. \\
\hline & $\mathrm{I}$ & 'Cause you were talking about a proof by contradiction, right? \\
\hline & $\mathrm{S}$ & Yeah. \\
\hline & I & $\begin{array}{l}\text { So before you even start doing any work, what's the assumption that } \\
\text { you're gonna make in your proof? That very first line of your proof, what } \\
\text { are you gonna assume to be happening in this problem that makes it a } \\
\text { proof by contradiction? }\end{array}$ \\
\hline & $\mathrm{T}$ & $\begin{array}{l}\text { So you're gonna assume there's a point in } \mathrm{X} \text { that's not in the closure of } \mathrm{A} \\
\text { [static point to } \bar{A}=X_{\text {] }} \text {. }\end{array}$ \\
\hline & $\mathrm{S}$ & And then that would mean that $\mathrm{A}$ is not dense in X. \\
\hline \multirow[t]{5}{*}{$42: 53$} & I & $\begin{array}{l}\text { Right. Yeah, so when you do a proof by contradiction, you suppose the } \\
\text { hypothesis is true, right? So suppose that for each open set } \mathrm{O} \text { in } \mathrm{T} \text {, we } \\
\text { have that the intersection is nonempty, but then assume that } \mathrm{A} \text { is not } \\
\text { dense in X. So we assume that part is false. }\end{array}$ \\
\hline & $\mathrm{T}$ & Yeah. \\
\hline & $\mathrm{S}$ & $\begin{array}{l}\text { So then that's our, how we re assuming that, that we're letting } \mathrm{x} \text { be in } \mathrm{X} \\
\text { but not in A? }\end{array}$ \\
\hline & I & $\begin{array}{l}\text { So we know that the closure of } \mathrm{A} \text { has to be a subset of } \mathrm{X} \text {, right? Because } \\
\text { everything in the closure is gonna be in the space. }\end{array}$ \\
\hline & $\mathrm{T}$ & Yeah. \\
\hline
\end{tabular}




\begin{tabular}{|c|c|c|}
\hline & $\mathrm{S}$ & So we're saying that... [writing "Let $x \in X$ and $x \notin A$ "] Like that? \\
\hline & I & $\begin{array}{l}\text { And why can we do that? Why can we choose that } \mathrm{x} \text { ? How do we know } \\
\text { that one exists in this proof? }\end{array}$ \\
\hline & $\mathrm{T}$ & Because we tried to assume the ... false... \\
\hline & $\mathrm{S}$ & The opposite. [points from up to down, indicating a switch] \\
\hline & $\mathrm{T}$ & The opposite. \\
\hline & I & The opposite of what? \\
\hline & $\mathrm{S}$ & This. [underlines "then $\mathrm{A}$ is dense in $\mathrm{X}$ "] \\
\hline & $\mathrm{T}$ & $A$ is dense in $\mathrm{X}$ \\
\hline & I & $\begin{array}{l}\text { OK, so we're assuming } A \text { is not dense in } \mathrm{X} \text {, so that's how we know that } \\
\text { that } \mathrm{x} \text { exists. [long pause] }\end{array}$ \\
\hline \multirow[t]{11}{*}{ 44:36 } & $\mathrm{S}$ & 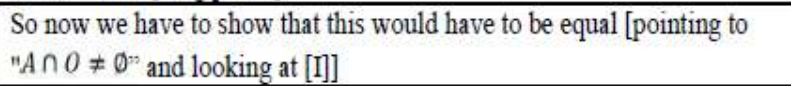 \\
\hline & I & So... OK. \\
\hline & $\mathrm{T}$ & 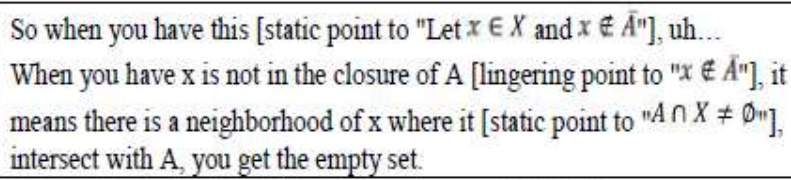 \\
\hline & $\mathrm{S}$ & 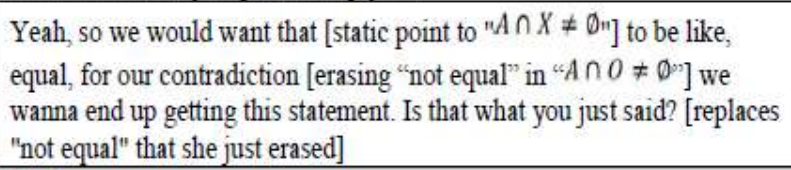 \\
\hline & $\mathrm{T}$ & Yeah. \\
\hline & I & I think he's telling you the next step in your proof. \\
\hline & $\mathrm{S}$ & OK You wanna just write it? \\
\hline & $\mathrm{T}$ & It doesn't seem right... \\
\hline & I & $\begin{array}{l}\text { Go ahead and write what you're thinking, and then we can assess it from } \\
\text { there. }\end{array}$ \\
\hline & $\mathrm{S}$ & $\begin{array}{l}\text { Yeah, I have some trouble thinking when people say things out loud } \\
\text { sometimes. }\end{array}$ \\
\hline & $\mathrm{T}$ & $\begin{array}{l}\text { [tracing point along "Let } x \in X \text { and } x \in \bar{A} \text {," writes " } " \exists N \in N_{x} \text { s.t. } \\
N \cap A=\emptyset \text { " then points to what he wrote while reading silently] So it, so } \\
\text { when we have this [static point to } N \cap A=\emptyset \text { ], it already contradicts with } \\
\text { this assumption. [static point to } O \in \mathcal{T}_{\text {] }}\end{array}$ \\
\hline \multirow[t]{6}{*}{$46: 07$} & I & How does that contradict? \\
\hline & $\mathrm{T}$ & $\begin{array}{l}\text { Because now you have an open set that, intersect with A, gives you, uh, } \\
\text { empty set. [static point either to } N \cap A=\emptyset \text { or to Stacey's first diagram] }\end{array}$ \\
\hline & I & Is $\mathrm{N}$ an open set? \\
\hline & $\mathrm{T}$ & No. It's a neighborhood. \\
\hline & I & It's a neighborhood? So where is the open set coming from. \\
\hline & $\mathrm{S}$ & It's within the neighborhood? [looking at [I]] \\
\hline
\end{tabular}

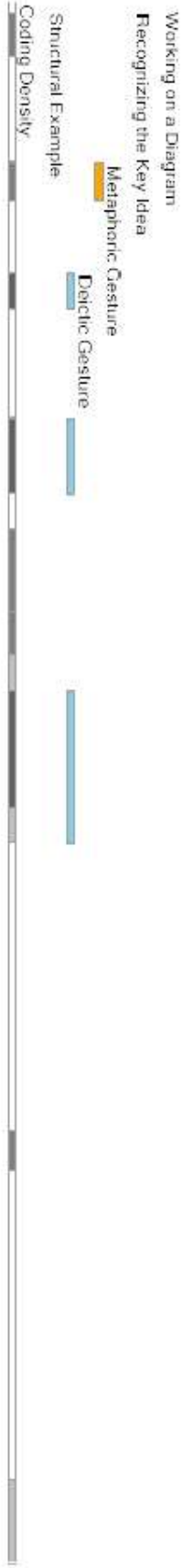




\begin{tabular}{|c|c|}
\hline I & OK. So because $\mathrm{N}$ is a neighborhood, it contains an open set. \\
\hline $\mathrm{T}$ & Yeah. \\
\hline I & OK. \\
\hline S & $\begin{array}{l}\text { So there's } \mathrm{O} \text {, subset of } \mathrm{N} \text {, whose intersection with } \mathrm{A} \text { is equal to the } \\
\text { empty set. }\end{array}$ \\
\hline I & I agree with that. \\
\hline $\mathrm{T}$ & 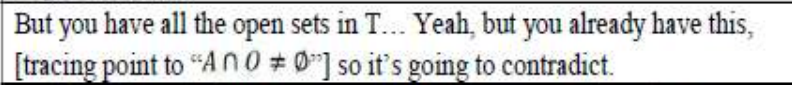 \\
\hline I & $\begin{array}{l}\text { Yeah. So that's it, right? We assumed that A was not dense in X, so } \\
\text { there's a neighborhood that has an empty intersection with A, so there } \\
\text { has to be an open set inside that neighborhood that has an empty } \\
\text { intersection with A. }\end{array}$ \\
\hline $\mathrm{T}$ & $\mathrm{Oh} !$ \\
\hline I & But we know that that can't happen. \\
\hline $\mathrm{S}$ & [writes "So $\exists 0 \subset N_{\text {s.t. }} O \cap A=\emptyset$ "] \\
\hline $\mathrm{T}$ & 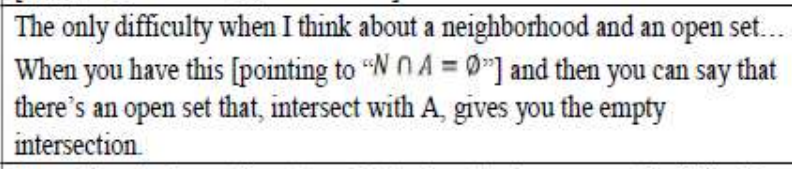 \\
\hline I & So making the jump from the neighborhood to the open set is difficult? \\
\hline $\mathrm{T}$ & $\begin{array}{l}\text { Yeah. Isn't the neighborhood [static point to } N \cap A=\emptyset \text { ] the collection of } \\
\text { open sets? [dynamic gesture, shaping his hands to trace the outline of a } \\
\text { ball in the air] }\end{array}$ \\
\hline I & It just contains an open set. \\
\hline $\mathrm{T}$ & It contains an open set. \\
\hline I & It doesn't necessarily have to be a union of them. \\
\hline I & $\begin{array}{l}\text { So if we have, let's say, what this is telling me is, [drawing] if this is my } \\
\text { set A, let's say that I have a neighborhood over here that's disjoint from } \\
\text { A. It's a neighborhood, so it contains an open set, let's call this Ox, and } \\
\text { put x in here... }\end{array}$ \\
\hline $\mathrm{S}$ & At least one, but it can contain more? \\
\hline I & $\begin{array}{l}\text { Right. It can contain as many as you want, but it has to have at least one } \\
\text { open set inside it. }\end{array}$ \\
\hline $\mathrm{T}$ & Yeah. \\
\hline I & $\begin{array}{l}\text { So if we have a neighborhood that's disjoint from A, the neighborhood } \\
\text { contains an open set... }\end{array}$ \\
\hline $\mathrm{T}$ & Oh! So... \\
\hline I & The open set's gonna be farther away from A, probably. \\
\hline $\mathrm{T}$ & Yeah. \\
\hline I & So that intersection definitely should be empty too. \\
\hline $\mathrm{T}$ & This is so obvious! \\
\hline S & $\begin{array}{l}\text { So then we just say, like if we're actually writing this proof out, we write } \\
\text { that line [tracing point along the last line of the proof on the board], and }\end{array}$ \\
\hline
\end{tabular}


we say "Thus, by contradiction, this statement..."

\begin{tabular}{|l|l|l}
\hline $49: 00$ & I & $\begin{array}{l}\text { Mhmm. Yeah, so we found an open set whose intersection with } \mathrm{A} \text { is } \\
\text { empty, but by assumption, it's not, so we contradicted. }\end{array}$ \\
\hline
\end{tabular}

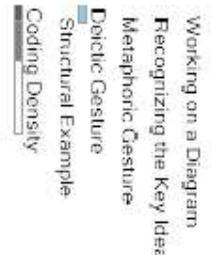

